\title{
SYMBOL-BY-SYMBOL MAP DEMODULATION OF CPM SIGNALS TRANSMITTED ON RAYLEIGH FLAT-FADING CHANNELS
}

\author{
by \\ Michael J. Gertsman. B.A.Sc. \\ A thesis submitted to \\ the Faculty of Graduate Studies and Research \\ in partial fulfillment of the requirements for the degree of \\ Master of Engineering \\ Department of Systems and Computer Engineering \\ Faculty of Engineering \\ Carleton University \\ Ortawa, Ontario \\ December, 1996 \\ (c) copyright \\ 1996, Michael J. Gertsman
}




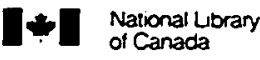

Acquistions and Bibliographic Services Branch 395 Wellungton Street Oriawa. Ontano
Kif onie
Sibliotheque nationale du Canaca

Direction des acquisitions et des services bibliographiques 395. vue Wethington KiA ONe
The author has granted an irrevocable non-exclusive licence allowing the National Library of Canada to reprociuce, loan, distribute or sell copies of his/her thesis by any means and in any form or format, making this thesis available to interested persons.
L'auteur a accordé une licence irrévocable et non exclusive permettant à la Bibliothèque nationale du Canada de reproduire, prêter, distribuer ou vendre des copies de sa thèse de quelque manière et sous quelque forme que ce soit pour mettre des exemplaires de cette thèse à la disposition des personnes intéressées.

L'auteur conserve la propriété du droit d'auteur qui protège sa thèse. Ni la thèse ni des extraits substantiels de celle-ci ne doivent être imprimés ou autrement reproduits sans son autorisation.

\section{Canadä}


Now Micheel Gertimin

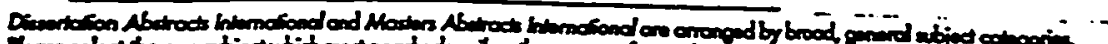

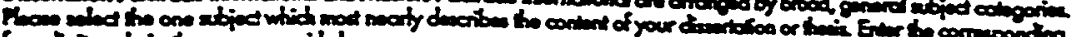
burdot cods in the spos provided

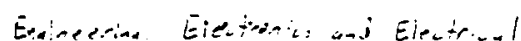
miation

\section{JI5Tाप UMI}

\section{Sobipd Cologories}

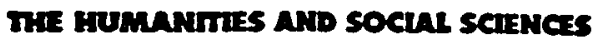
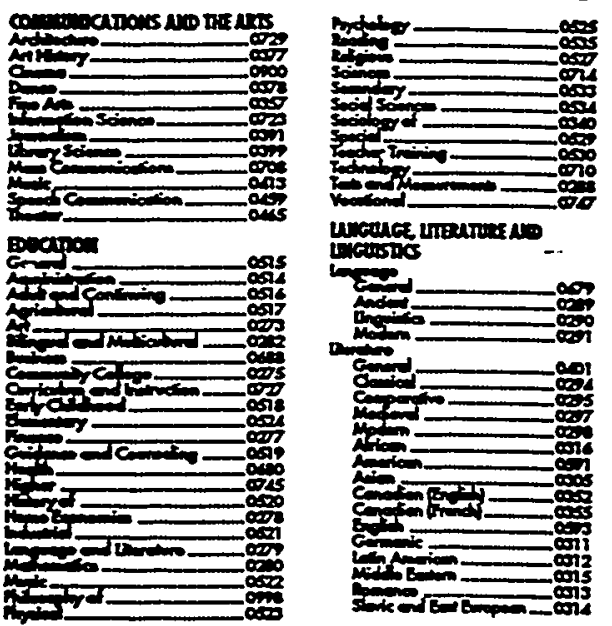

umace umarmas inctoris
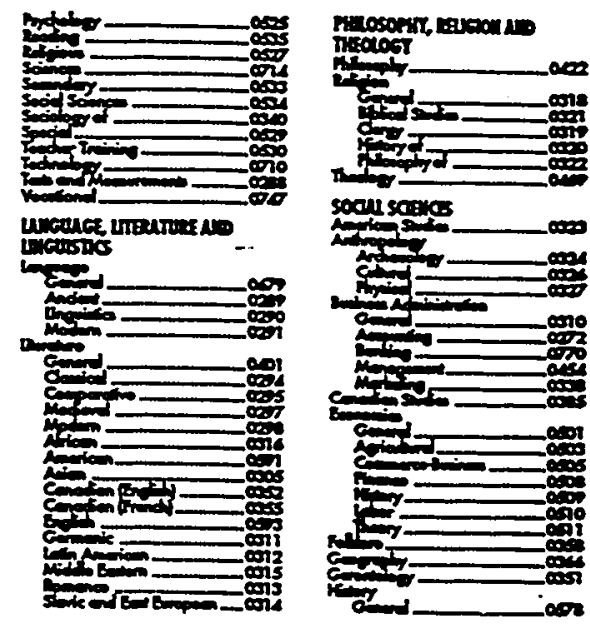

\section{TIE SCIENGSS AND ENGINEERINO}

\section{merens soses}

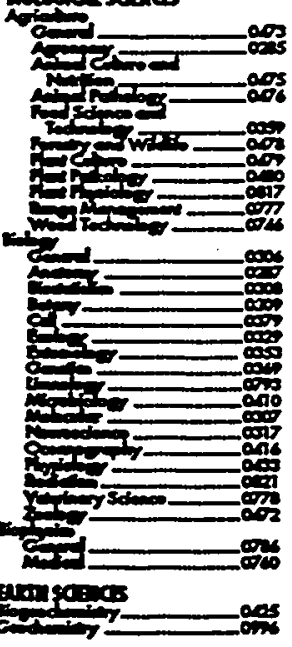

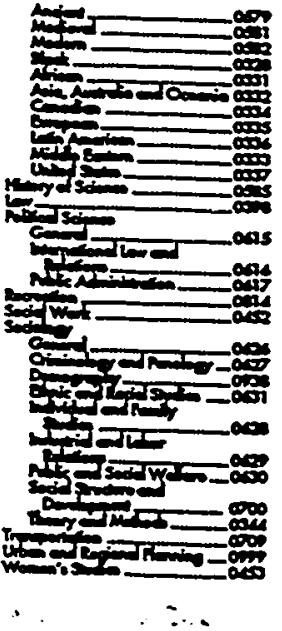

${ }_{n=1}^{2}$ miscas sosas prosina

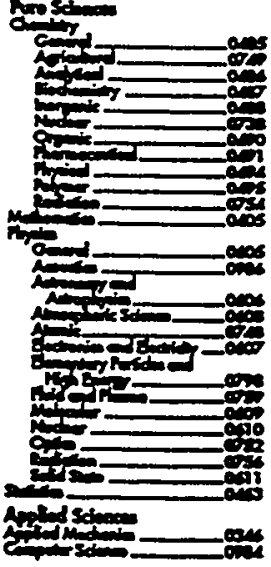

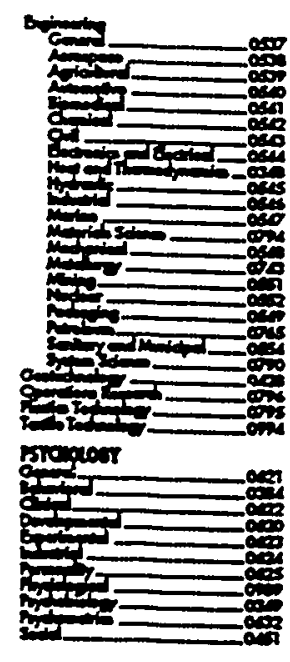


The undersigned hereby recommend to the faculty of Graduate Srudies and Research acceptance of the thesis, "SYMBOL.BY.SYMBOL MAP DEMODULATION OF CPM SIGNALS TRANSMITTED ON RAYLEIGH FLAT-FADNG CHANNELS", submitted by Michael J. Gertsman, B.A.Sc. in partial fulfillment of the requirements for the degree of Master of Enginecring.
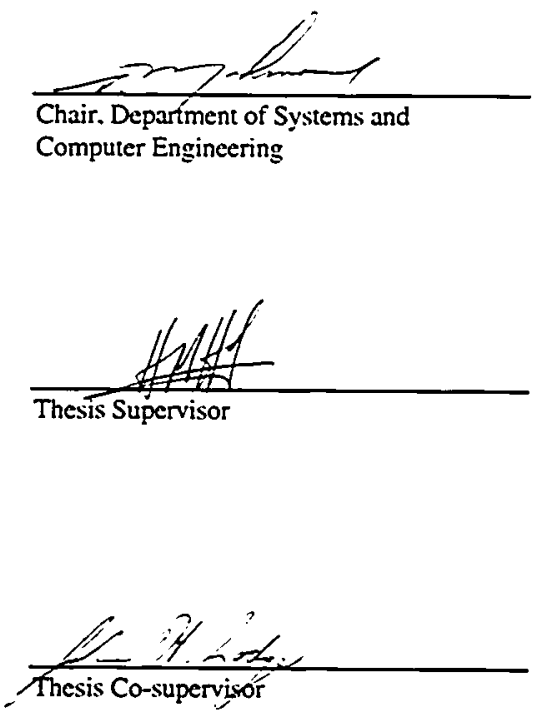

Deparment of Systems and Computer Engineering

Faculty of Engineering

Carleton University

Ottawa. Ontario

December, 1996 


\begin{abstract}
Demodulation using the symbol-by-symbol MAP algorithm is presented. The algorithm is derived for the case of CPM signals transmitted over Rayleigh flat-fading channels and a corresponding receiver structure is specinied. It is shown that the MAP algorithm requires computing. for each trellis branch. the sum of the products of the weights of all paths through the trellis which pass through that branch and that this generic computational problem can be efficiently solved by an approach that uses a forward and backward recursion through the trellis. Simulation results are presented which show both the hard and soft decision performance of the MAP receiver to be robust even in the presence of fade rates of up to $30 \%$ of the symbol rate. The MAP algorithm is also shown to procuce accurate BER estimates and to be amenable to reduced state implementation. The application of the receiver concept to QPSK signals is also discussed and then evaluated via simulation.

The concept of joint demodulation and decoding using iterative processing techniques is introduced. It is shown that the MAP receiver is well suited for iterative processing applications due to its use of a priori symbol probabilities and its production of optimal soft decisions. Simulation results for the reception of QPSK show that the BER performance of the iterative MAP receiver can approach that of a receiver operating with perfect knowledge of the fading process.
\end{abstract}




\section{Acknowledgments}

I would like to thank Dr. John Lodge of the Communications Research Centre (CRC) for his motivatung outlook. patience, and ongoing suppor.

I dedicate this thesis to my wife Sophia who gave so much of herself throughout my graduate studies program. Her unfailing suppor. understanding. encouragement and occasional prodding. were instrumental in allowing me to complete this work. 
Table Of Contents

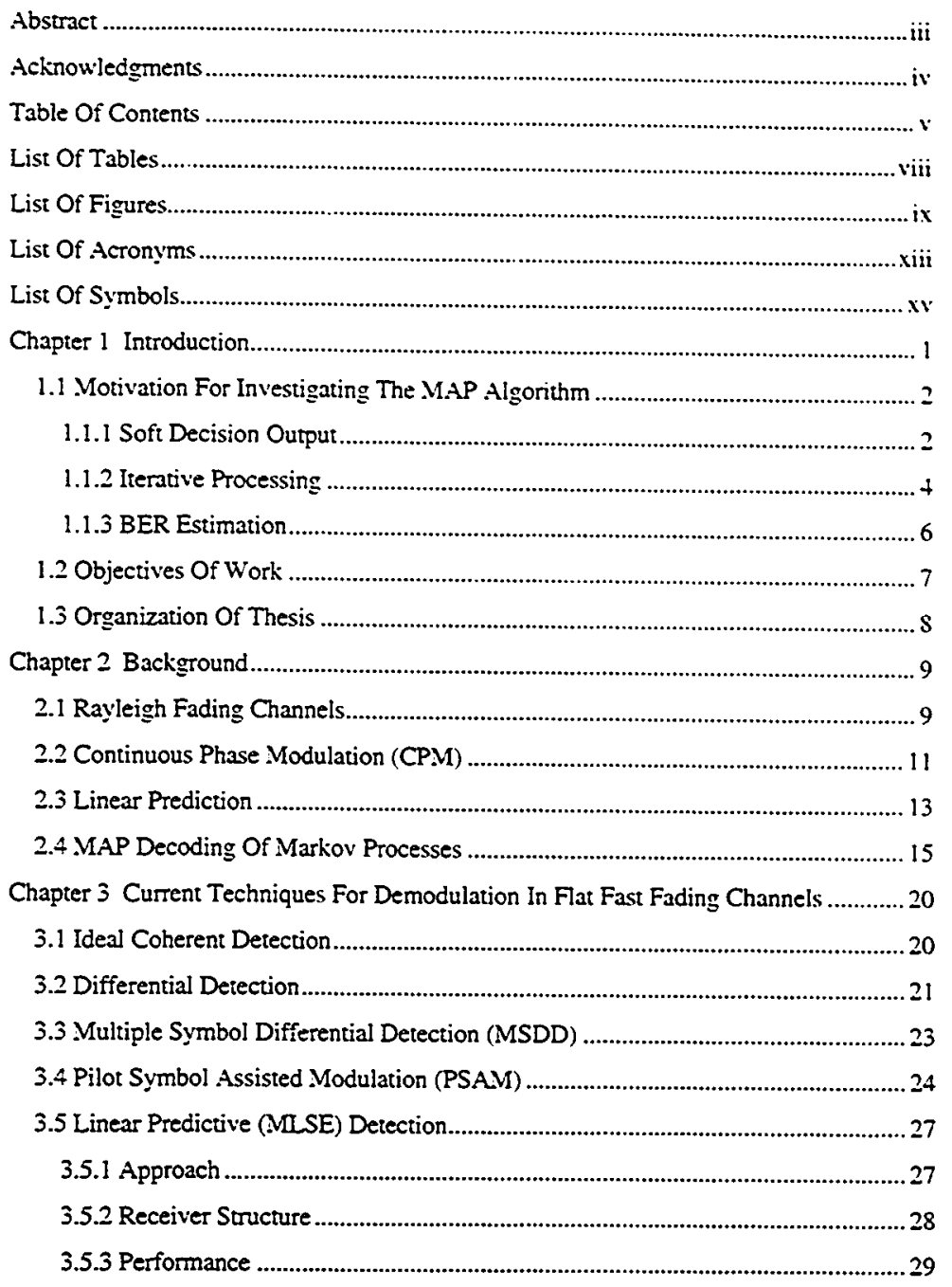




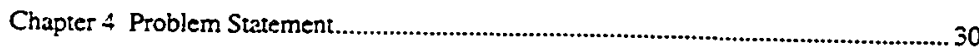

4.I Optimal Soft Decisions In Fast Fading ................................................................30

4.2 Disadvantages Of Current Approaches ............................................................... 30

4.3 Advantages Of Symbol-By-Symbol MAP Demodulation .................................... 31

Chapier 5 Symbol-By-Symbol MAP Demodulation......................................................... 33

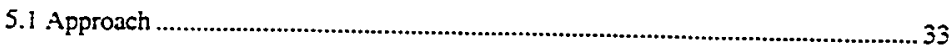

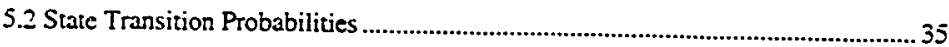

5.3 Efficient Computation Of The State Transition Probabilities................................. 37

5.4 Computation Of Soft Decisions ........................................................................40

5.5 Computation Of Probability Density................................................................. 41

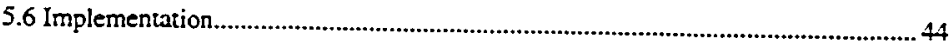

Chapter 6 Demodulation Performance .......................................................................47

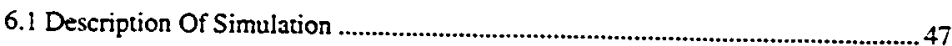

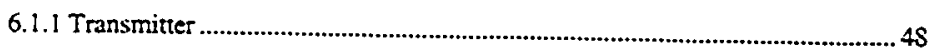

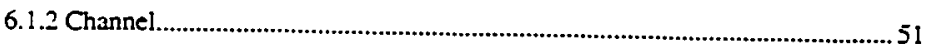

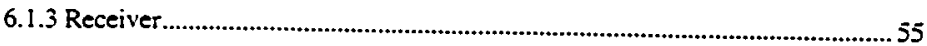

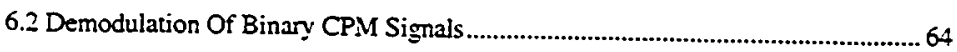

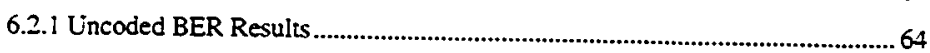

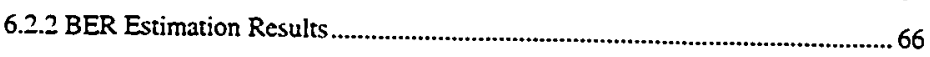

6.2.3 Coded BER Results ....................................................................................68

6.2.4 Average Mußual Information ......................................................................... 70

6.2.5 Reduced State Performance............................................................................ 74

6.2.6 Sensitivity To Channel Model..........................................................................77

6.2.7 Sensitivity To Expected Prediction Ertor......................................................... 79

6.3 Demodulation Of QPSK Signais......................................................................... \$2

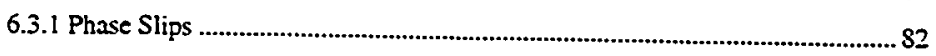

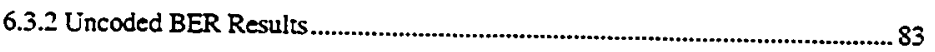

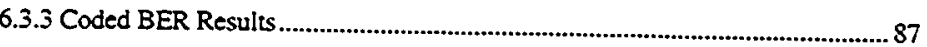

6.4 Summary Of Demodulation Results.................................................................... $\$ 9$

Chapter 7 Iterative MAP Processing For Joint Demodulation And Decoding ................. 91 
7.1 Goal Of The Irerative Processing ...................................................................... 91

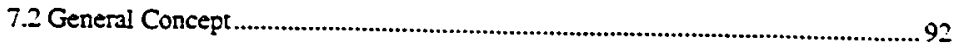

7.3 Structure Of Terative MAAP Receiver ....................................................................96

7.4 Soft Decision Reference Receiver ........................................................................99

7.5 Performance With QPSK Signals ................................................................. 100

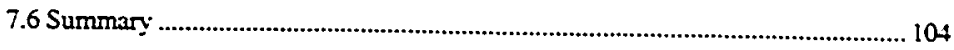

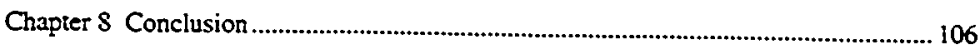

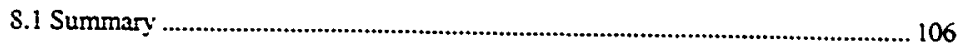

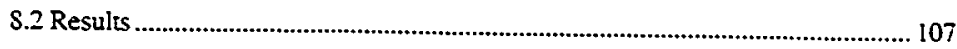

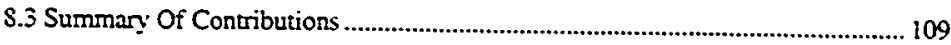

S 4 Suggestions For Future Research ................................................................. 109

Appendix A CPM Matched Filter Requirements......................................................111

tppendix B Simulation BER Confidence Intervals.................................................. 116

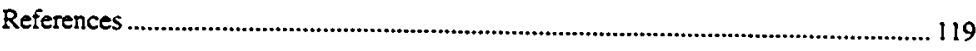




\section{List Of Tables}

Table 2- 1 The states traversed by paths passing through state 0 at time $t=2 \ldots \ldots \ldots \ldots \ldots . .16$

Table 6-I The set of reduced state test configurations.................................................... 75

Table 6-2 Performance Versus Pilot Symbol Rate At $E_{b} / N_{0}=10 \mathrm{~dB}$.............................. 83

Table 6-3 Symbol Probabilities For Phase Slip Error Run .............................................. \$6 


\section{List Of Figures}

Figure 1-1 A tracitional demodulator structure................................................................

Figure 1-2. An example of a system utilizing concatenated processing techniques. .......... 3

Figure 1-3 A receiver structure utilizing iterative M.AP decoding....................................5

Figure I - A receiver structure utilizing iterative M.AP demodulation/decoding...............6

Figure 2-1 A block diagram of the model for a Rayleigh fading channel with additive

white Gaussian noise (AWGN). WGN $(\sigma)$ represents a white Gaussian noise

process with variance $\sigma$.

Figure 2-2 The trellis of an example Markov process................................................. Is

Figure 3-I A simple differential detector...................................................................22

Figure 3-2 The BER performance of differential detection of differentially encoded

BPSK. QPSK and S-PSK at fade rates of $0.01 R$ and $0.05 R$................................... 23

Figure 3-3 At transmission system utilizing pilot symbol assisted modulation.................25

Figure 3-4 The PSAM transmitted symbol stream with one pilot symbol per $P-1$ data

symbols.................................................................................................................. 26

Figure 3-5 The structure of the Linear Predictive Receiver............................................ 29

Figure 5-1 A partial trellis for a binary system with 3 bits of memory............................. 34

Figure 5-2 (a) The trellis of the example Markov process. (b) The equivalent treilis when only those paths passing through the branch from state $S_{3}=0$ to state $S_{4}=0$ are considered

Figure 5-3 Statistically equivalent alternate transmission models (from [33])................42

Figure 5-4 The structure of the filter bank used by the MAP demodulator. .................... 45

Figure 6-1 A block diagram of the simulation. ...............................................................4

Figure 6-2 A block diagram of the simulated transmitter.............................................4 48

Figure 6-3 The (a) phase pulse and (b) power spectrum of N32FM. .............................50

Figure 6-4 The frame structure used for the MAP simulations........................................51

Figure 6-5 A block diagram of the simulated channel. ..................................................52

Figure 6-6 The impulse and frequency responses of the fading filter.............................54 
Figure 6-7 A block diagram of the simulared receiver. .55

Figure 6-8 A block diagram of the simulated MAP demodulator....................................57

Figure 6-9 A flowchar of the symbol-by-symbol MAP processing................................. 59

Figure 6-10 Predicted and actual filters for a fading rate of $0.3 R$ at $r=2$ samples $/$ symbol.62

Figure 6.11 The effect of predictor order on fit for a fading rate of $0.3 R$ and an $E_{s} / N_{0}$ of

$10 \mathrm{~dB}$ at $r=2$ samples/symbol.

Figure 6-12 Prediction error vs. predictor order for a fading rate of $0.3 R$ and an $E_{s} / N_{0}$ of

$10 \mathrm{~dB}$ at $r=2$ samples/symbol.

Figure 6-13 The BER performance of the MAP and MLSE demodulators as compared to that of differential detection for the reception of binary CPM signals. The fading rate is $0.05 R$

Figure 6-14 The BER performance of the MAP and MLSE demodulators as compared to that of differential detection for the reception of binary CPM signals. The fading rate is $0.3 R$

Figure 6-15 The BER estimation performance of the MAP demodulator for the reception of binary CPM signals as a function of window size. The fading rate is $0.05 R$ and the $E_{b} / N_{0}$ is $10 \mathrm{~dB}$

Figure 6-16 The coded and uncoded BER performance of the MAP and MLSE demodulators as compared to that of differential detection for the reception of binary CPM signals. The fading rate is $0.05 R$. Coded MISE results utilize hard decision inputs to the decoder while the coded MAP and differential detection results utilize soft decisions.

Figure 6-17 The coded and uncoded BER performance of the MAP and MLSE demodulators as compared to that of differential detection for the reception of binary CPM signals. The fading rate is $0.3 R$. Coded MLSE results urilize hard decision inputs to the decoder while the coded MAP and differential detection results utilize soft decisions

Figure 6-1S Comparisons of hard and sofi decision qualin............................................. 73

Figure 6-19 The reduced-state BER performance of the MAP demodulator. The fading rate is $0.05 R$. 
Figure 6-20 The reduced-state BER performance of the MIAP demodulator. The tading rate is $0.3 R$.

Figure 6-21 The sersitivity of the binany CPM BER performance of the M.AP demodulator to erroxs in the modelled AWGN noise level. The $E_{N} / N_{0}$ is $10 \mathrm{~dB}$. representing the case where tne effect of the noise dominates the pertiormance. The fading rate is $0.05 R$.

Figure 6-22 The sensitivity of the binary CPM BER pertormance of the MAP demodulator to errors in the modelled iading bandwidth. The $E_{b} / N_{0}$ is $25 \mathrm{~dB}$. representing the case where the effect of the fading dominates the performance. The fading rate is $0.3 R$.

Figure 6-23 The sensitivity of the binary CPM BER performance of the MAP demodulator to errors in the estimated squared prediction error $\left(r_{0} V_{i}\right)$. The $E_{N} / N_{0}$ is $25 \mathrm{~dB}$ ard the fading rate is $0.3 R$.

Figure 6-24 The BER performance of the MAP demodulator as compared to that of differential detection for the reception of QPSK signals. The fading rate is $0.05 R$. For the MAP simulations, pilot symbol rates of $1: S$ and $1: 16$ were used. For the differential detection simulations. the signal was differentially encoded.

Figure 6-25 An example of the QPSK symbol probabilities after a phase slip has occurred.

Figure 6-26 The coded and uncoded BER performance of the MAP demodulator for QPSK with a pilot symbol rate of $1: 8$ as compared to that of differential detection of differentially encoded QPSK. The fading rate is $0.05 R$.

Figure 6-27 The coded and uncoded BER performance of the MAP demodulator for QPSK with a pilot symbol rate of 1:16 as compared to that of differential detection of differentially encoded QPSK. The fading rate is $0.05 R$.

Figure 7-1 An example of a system utilizing coneatenated processing techniques. ........ 94

Figure 7-2 The structure of iterative MAP demodulation/decoding.

Figure 7-3 The equivalent channel model for the ideal channel state receiver. 
Figure 7-4 The BER performance of iterative MAP demodularion/decoding as compared to that of conventional differential detection and convolutional decoding for the reception of QPSK signals. Also shown is the performance of a fictitious reference receiver utilizing ideal channel state information. The fading rate is $0.05 R$ and the pilot symbol rate used in the MAP simulacions is $1: 8$. 101

Figure 7-5 The BER performance of iterative MAP demodulation/decoding as compared to that of conventional differential detection and convolutional decoding for the reception of QPSK signals. Also shown is the performance of a fictitious reference receiver utilizing ideal channel state information. The fading rate is $0.05 R$ and the pilot symbol rate used in the MAP simulations is $1: 16$

Figure A-1 A graphical representation of the symbols affecting the prediction error filter (PEF) calculation at $t=3$ for $k=2.5$ symbol yeriods. $\chi=1.5$ symbol periods and $r=2$ samples/symbol. 


\section{List Of Acronyms}

$\begin{array}{ll}\text { ANSI } & \text { American National Standards Institute } \\ \text { AWGN } & \text { Additive White Gaussian Noist } \\ \text { BER } & \text { Bit Error Rate } \\ \text { BPSK } & \text { Binary Phase Shift Keying } \\ \text { CPM } & \text { Continuous Phase Modulation } \\ \text { CRC } & \text { Canadian Communications Research Centre } \\ \text { CSI } & \text { Channel State Information } \\ \text { CW } & \text { Continuous Wave } \\ \text { DMC } & \text { Discrete Memoryless Channel } \\ \text { FEC } & \text { Forward Error Correction } \\ \text { FFT } & \text { Fast Fourier Transform } \\ \text { FIR } & \text { Finite Impulse Response } \\ \text { FM } & \text { Frequency Modulation } \\ \text { GSM } & \text { Global System for Mobile Communications } \\ \text { HD } & \text { Hard Decision } \\ \text { I } & \text { In-phase component of a complex baseband signal } \\ \text { M-ary PSK } & \text { M-phase Phase Shift Keying } \\ \text { MAP } & \text { Maximum a posteriori Probability } \\ \text { MLSE } & \text { Maximum Likelihood Sequence Estimation } \\ \text { MSDD } & \text { Multiple Symbol Differential Detection } \\ \text { MSK } & \text { Minimum Shift Keying } \\ \text { N32FM } & \text { Nyquist 3-2 Pulse Shaped Frequency Modulation } \\ \text { PC } & \text { IBM-compatible Personal Computer } \\ \text { PEF } & \text { Prediction Error Filter } \\ \text { PSAM } & \text { Pilot Symbol Aided Modulation } \\ \end{array}$




$\begin{array}{ll}\text { PSI } & \text { Pilot Symbol Insertion } \\ \text { PSK } & \text { Phase Shift Keying } \\ \text { Q } & \text { Quadrature component of a complex baseband signal } \\ \text { QPSK } & \text { Quadrature Phase Shift Keying. also called 4-PSK } \\ \text { SD } & \text { Soft Decision } \\ \text { SER } & \text { Symbol Eror Rate } \\ \text { SNR } & \text { Signal To Noise Ratio } \\ \text { SOVA } & \text { Soft Ourput Viterbi Algorithm } \\ \text { TCM } & \text { Trellis Coded Modulation } \\ \text { TDMA } & \text { Time Division Multiple Access } \\ \text { TTIB } & \text { Transparent Tone In Band } \\ \text { WGN } & \text { White Gaussian Noise } \\ \text { 8-PSK } & \text { S-phase Phase Shift Keying }\end{array}$




\section{List Of Symbols}

\begin{tabular}{|c|c|}
\hline$A$ & $\begin{array}{l}\text { the set of all state transitions for which the input symbol is a hypothesized } \\
\text { symbol } q\end{array}$ \\
\hline$a_{t}$ & $\begin{array}{l}\text { the lth coefficient of a linear prediction model relating the output of the } \\
\text { system at a given time to the past outputs of the system }\end{array}$ \\
\hline$a_{i}^{L}$ & the $i$ th coefficient of the $L$ th-order linear predictor \\
\hline$B_{j}(k)$ & $\begin{array}{l}\text { the output at time } j \text { of a prediction error filter combined with the removal } \\
\text { of the modularion corresponding to hypothesis } k\end{array}$ \\
\hline$b_{j}$ & $\begin{array}{l}\text { the jth coefficient of a linear prediction model relating the output of the } \\
\text { system at a given time to the past inputs of the system }\end{array}$ \\
\hline$C_{t}\left(m^{\prime} . m\right)$ & $\begin{array}{l}\text { the set of hypotheses }\{k\} \text { that traverse the trellis branch between states } \\
S_{t-1}=m^{\prime} \text { and } S_{t}=m\end{array}$ \\
\hline$D$ & the number of stages in an iterative processing system \\
\hline$d$ & the stage number in an iterative processing system \\
\hline$E[\cdot]$ & an expected value \\
\hline$E_{b}$ & the energy per bit \\
\hline$E_{s}$ & the energy per symbol \\
\hline$f$ & the frequency in $\mathrm{Hz}$ \\
\hline$f_{c}$ & the carrier frequency in $\mathrm{Hz}$ \\
\hline$G$ & a gain parameter for a linear prediction model \\
\hline$g(\tau)$ & the instantaneous frequency pulse shape \\
\hline$h$ & the modulation index \\
\hline$H(f)$ & the frequency response of the fading process filter \\
\hline$I(W: Z)$ & the average mutual information between two random variables $W$ and $Z$ \\
\hline$I_{h d}(W ; Z)$ & $\begin{array}{l}\text { the average mutual information of the hard decisions } Z \text { corresponding to } \\
\text { inputs } W\end{array}$ \\
\hline$I_{s d}(W ; Z)$ & $\begin{array}{l}\text { the average mutual information of the soft decisions } Z \text { corresponding to } \\
\text { inputs } W\end{array}$ \\
\hline
\end{tabular}




\footnotetext{
$I_{\text {fit }}(n)$ the improvement factor which relates the information at the output of a MAP filter to that at its input

$I_{\text {sit., }}(n)$ the improvement factor for the $p$ th iteration which relates the information at the output of a MAP filter to chat at its input

$J$ the number of zeroes in a linear predictor

$K$ the combined memory, in symbol periods. of the modulator and the channel

$k$ the hypothesis

$k_{i} \quad$ the $/$ th reflection coefficient of a linear predictor

$L \quad$ the predictor order (for an all-pole linear predictor)

$L_{n} \quad$ the likelihood ratio for the bit transmitted at time $n$

$L_{d \mathrm{~m}}(n)$ the likelihood ratio output from the demodulator for the bit at time $n$

$L_{f l i}(n)$ the likelihood ratio output from the MAP filter for the bit at time $n$

-. $\quad L_{d m, p}(n) \quad$ the likelihood ratio for the bit at time $n$ which is to be input to the MAP filter on the $p$ th iteration

$L_{d m, p}^{\prime}(n) \quad$ the likelihood ratio for the bit at time $n$ which is output from the demodulator on the pth iteration

$M \quad$ the number of states in the trellis

$m^{\prime} \quad$ a starting stare for a symbol interval

$m \quad$ an ending state for a symbol interval

$\mathbf{M}(k)$ the sequence of state transitions through the trellis corresponding to hypothesis $k$

$\mathbf{M}_{i}(k)$ the $i$ th element of the path through the trellis corresponding to hypothesis $k$

$N \quad$ the length of the data block, in symbol periods

$N$. the number of independent simulation runs for a given point

$N_{0} \quad$ the single-sided additive white gaussian noise density

$n \quad$ a discrete time index

$n$, the jth complex baseband sample of the additive white gaussian noise
} 


\begin{tabular}{|c|c|}
\hline$P$ & the pilot symbol spacing \\
\hline$P_{\text {aur }}$ & an augmented $a$ priori probability to be input to the demodulator \\
\hline$P_{\mathrm{dem}}$ & a probability output from the demodulator \\
\hline$P_{\text {fit: }}$ & a probability output from the MAP filter \\
\hline $\bar{P}_{c}$ & the average probability of error \\
\hline$P_{e}(j)$ & the probability of error for run $j$ \\
\hline $\operatorname{Pr}(\cdot)$ & a probability \\
\hline$p$ & the iteration number \\
\hline$p(\cdot)$ & a probability density function \\
\hline$p_{y, t}: t, 1$ & $\begin{array}{l}\text { the probability distribution function of the received channel samples } \\
\text { conditioned on the input and the previously received samples }\end{array}$ \\
\hline$Q$ & the number of data symbols ( $Q$-ary modulation) \\
\hline$q$ & one of the $Q$-ary input symbols \\
\hline$R$ & the symbol rate in symbols per second \\
\hline $\mathbf{R}$ & $\begin{array}{l}\text { the Toeplitz covariance matrix whose elements correspond to the } \\
\text { composite fading plus noise power spectral density function } S^{\prime}(f)\end{array}$ \\
\hline $\mathbf{R}^{a}$ & a vector of the $\log$ likelihood ratios of the a priori information \\
\hline $\mathbf{R}_{p}^{a}(d)$ & $\begin{array}{l}\text { a vector of the log likelihood ratios of the augmented a priori information } \\
\text { input to stage } d \text { on the pth iteration }\end{array}$ \\
\hline $\mathbf{R}^{c}$ & a vector of the composite log likelihood ratios \\
\hline $\mathbf{R}^{\boldsymbol{c}}(\boldsymbol{d})$ & $\begin{array}{l}\text { a vector of the composite log likelihood ratios after the processing of } \\
\text { stage } d\end{array}$ \\
\hline $\mathbf{R}_{p}^{c}(d)$ & $\begin{array}{l}\text { a vector of the composite log likelihood ratios after the processing of } \\
\text { stage } d \text { on the pth iteration }\end{array}$ \\
\hline $\mathbf{R}^{e}(d)$ & $\begin{array}{l}\text { a vector of the log likelihood ratios of the extrinsic information after the } \\
\text { processing of stage } d\end{array}$ \\
\hline $\mathbf{R}_{p}^{*}(d)$ & $\begin{array}{l}\text { a vector of the log likelihooci ratios of the extrinsic information after the } \\
\text { processing of stage } d \text { on the } p \text { th iteration }\end{array}$ \\
\hline $\mathbf{R}^{i}$ & a vector of the log likelihood ratios of the intrinsic information \\
\hline
\end{tabular}




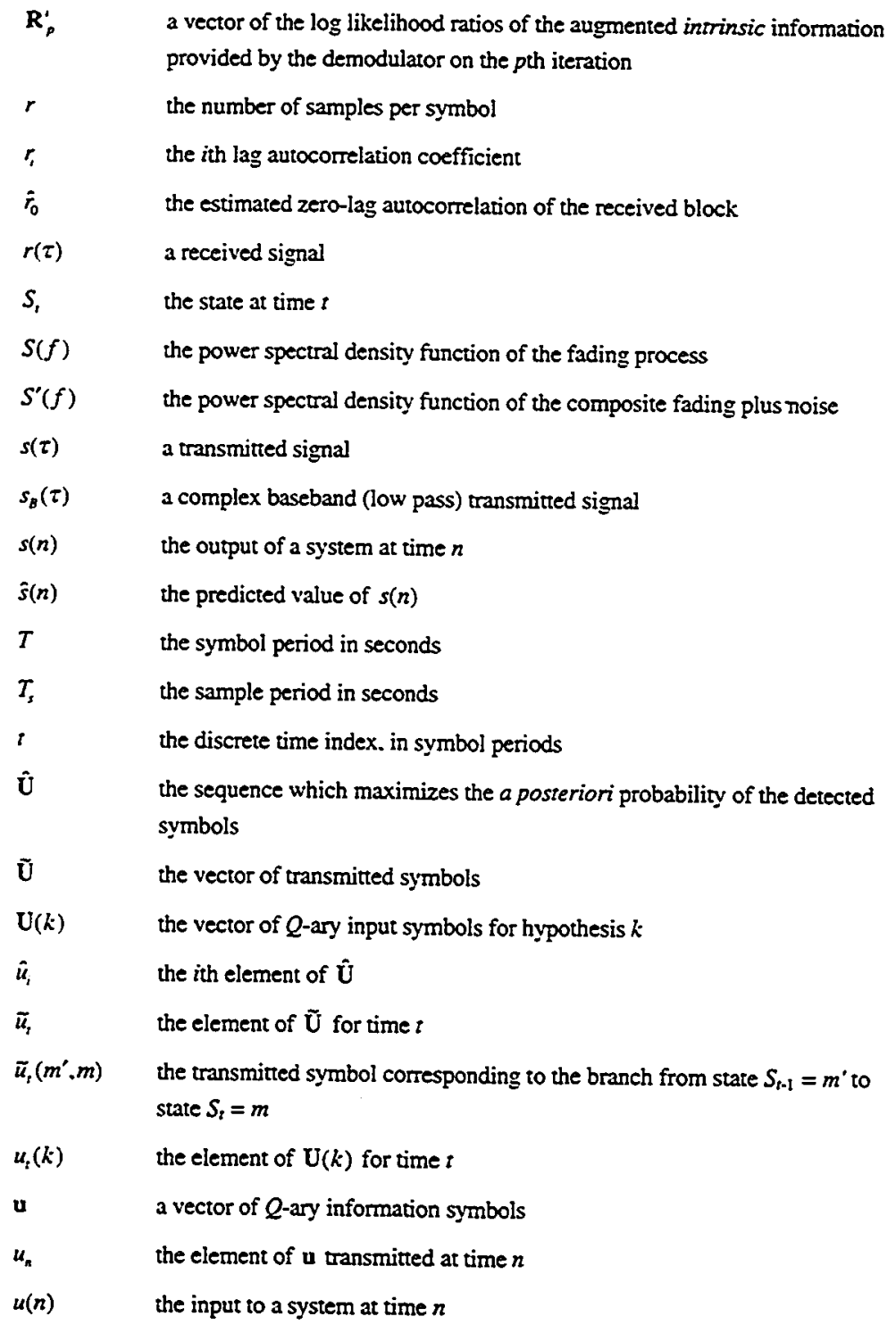




\begin{tabular}{|c|c|}
\hline$V_{i}$ & $\begin{array}{l}\text { the expected normalized squared prediction error for a ith-order linear } \\
\text { predictor }\end{array}$ \\
\hline$v_{j}$ & $\begin{array}{l}\text { the jh sample of the received signal after removal of the phase change } \\
\text { introduced by the channel }\end{array}$ \\
\hline WGN $(\sigma)$ & a white gaussian noise process with variance $\sigma$ \\
\hline$w$ & a random variable \\
\hline$w$ & the ith outcome of $W$ \\
\hline$\overline{\mathbf{x}}$ & the vector of transmitred samples \\
\hline $\mathbf{X}(k)$ & the vector of transmitred samples for hypothesis $k$ \\
\hline$x_{n}(k)$ & the element of $\mathrm{X}(k)$ for time $n$ \\
\hline$x_{j}$ & the transmitted sample for the jth symbol \\
\hline $\mathbf{Y}$ & the vector of received samples \\
\hline $\mathbf{Y}^{\prime}(k)$ & $\begin{array}{l}\text { the vector of received samples after the modulation corresponding to } \\
\text { hypothesis } k \text { has been removed }\end{array}$ \\
\hline$Y_{i}^{j}$ & the sequence consisting of the ith through jth element of $\mathrm{Y}$ \\
\hline$y(\tau)$ & the received signal prior to sampling \\
\hline$y_{j}^{\prime}(k)$ & $\begin{array}{l}\text { the jth received sample after the hypothesized modulation has been } \\
\text { removed }\end{array}$ \\
\hline$y_{j, j-1}^{\prime}(k)$ & the predicted channel sample for hypothesis $k$ at time $j$ \\
\hline$y_{n}$ & the element of $\mathrm{Y}$ for time $r$ \\
\hline$y_{n}(k)$ & the received symbol sample for hypothesis $k$ at time $n$ \\
\hline$y_{n}^{\prime}(k)$ & $\begin{array}{l}\text { the received symbol sample for hypothesis } k \text { at time } n \text { after the modulation } \\
\text { has been removed }\end{array}$ \\
\hline$y_{j}$ & the received sample for jith symbol \\
\hline$y_{r}(k)$ & $\begin{array}{l}\text { the set of data comprised of the samples of the received signal up to and } \\
\text { including time } t \text { and the sequence of transmitted symbol samples for } \\
\text { hypothesis } k\end{array}$ \\
\hline$z$ & a random variable \\
\hline$z_{j}$ & the $j$ th outcome of $Z$ \\
\hline
\end{tabular}




\begin{tabular}{|c|c|}
\hline$\alpha_{t}(m)$ & $\begin{array}{l}\text { the sum of the products of the weights along all paths which terminate in } \\
\text { state } m \text { at time } \text { ? }\end{array}$ \\
\hline$\tilde{\alpha}_{i}(m)$ & $\begin{array}{l}\text { the joint probability that the process was in state } m \text { at time } t \text {. and the } \\
\text { sequence of observations up to and including time } t\end{array}$ \\
\hline$\beta_{i}(m)$ & $\begin{array}{l}\text { the sum of the products of the weights along all paths to the end of the } \\
\text { trellis which originate in state } m \text { at time } t\end{array}$ \\
\hline $\bar{\beta}_{t}(m)$ & $\begin{array}{l}\text { the conditional probability of the sequence of observations after time } t \text {. } \\
\text { given that the process was in state } m \text { at time } t\end{array}$ \\
\hline$\chi$ & the predictor length in symbol periods \\
\hline$\Delta \hat{\varphi}_{j}$ & the hypothesized differentially encoded phase shift \\
\hline$\Lambda_{s}^{k}(\mathbf{Y})$ & the likelihood index for hypothesis $k$ of a length $N$ sequence \\
\hline$\gamma_{r}\left(m^{\prime}, m\right)$ & $\begin{array}{l}\text { the weights associated with the trellis branch corresponding to the } \\
\text { transition from state } m^{\prime} \text { to state } m \text { during symbol interval } l\end{array}$ \\
\hline$\gamma_{?}^{\prime}\left(m^{\prime}, m\right)$ & the trellis branch weights with the common factors removed \\
\hline$\gamma_{i}^{\prime}(k)$ & the filter bank outputs for hypothesis $k$ \\
\hline $\bar{\gamma}_{f}\left(m^{\prime}, m\right)$ & $\begin{array}{l}\text { the conditional joint probability that the process was in state } m \text { at time } t \\
\text { given that the process was in state } m^{\prime} \text { at time } t-1\end{array}$ \\
\hline$\varepsilon_{1}$ & the total error for a linear predictor of order $i$ \\
\hline$\kappa$ & the pulse length in symbol periods \\
\hline$\overline{\hat{\lambda}}_{s}(m)$ & $\begin{array}{l}\text { the joint probability that the process was in state } m \text { at time } t \text {. and the } \\
\text { sequence of observations } \mathbf{Y}\end{array}$ \\
\hline$\phi_{0}$ & an initial phase \\
\hline$\phi(\tau . u)$ & the time varying phase of a CPM signal \\
\hline$\mu_{t}$ & $\begin{array}{l}\text { the probability that the bit transmitted at time } t \text { was a } 1 \text { (the bit soft } \\
\text { decision) }\end{array}$ \\
\hline$\mu_{\mathrm{dem}}(n)$ & $\begin{array}{l}\text { the probability at the output of the demodulator that the data bit at dime } n \\
\text { is a } 1 \text { : i.e.. the soft decision }\end{array}$ \\
\hline$\mu_{d m,},(n)$ & $\begin{array}{l}\text { the probability at the output of the demodulator for the } p \text { th iteration that } \\
\text { the data bit at time } n \text { is a 1: i.e., the soft decision for the pth iteration }\end{array}$ \\
\hline
\end{tabular}




\begin{tabular}{|c|c|}
\hline$\mu_{j, t}(n)$ & $\begin{array}{l}\text { the probability at the output of the MAP filter that the data bit at time } n \text { is } \\
\text { a I }\end{array}$ \\
\hline$\eta_{j}$ & the metric input to the Viterbi algorithm for the jth symbol \\
\hline$\rho$ & the ith lag normalized autocorrelation coefficient \\
\hline$\sigma_{i}\left(m^{\prime} . m\right)$ & $\begin{array}{l}\text { the sum of the products of the weights of all paths which pass through the } \\
\text { branch delineated by } S_{i-1}=m^{\prime} \text { and } S_{i}=m\end{array}$ \\
\hline$\sigma_{j i j-1}^{2}(k)$ & the variance of the predicted channel sample for time $j$ \\
\hline $\bar{\sigma}_{z}\left(m^{\prime}, m\right)$ & $\begin{array}{l}\text { the joint probability of a branch delineated by } S_{t-1}=m^{\prime} \text { and } S_{t}=m \text { being } \\
\text { traversed. and the sequence of observations } Y\end{array}$ \\
\hline$\tau$ & a continuous time index \\
\hline$\xi_{n}$ & the fading process sample at time $n$ \\
\hline$\xi_{j}$ & the $j$ th sample of the multiplicative fading process \\
\hline$\psi$ & the estimated block bit етог rate \\
\hline
\end{tabular}




\section{Chapter 1}

\section{Introduction}

Interest in terrestrial mobile communications is expanding rapidly. While studies vary considerably in their predictions [1]. it is clear that contention for spectrum allocation will necessitate the increasingly efficient usage of bandwicth [2] while cost. size and interference considerations place similar requirements on signal power. To meet these and other challenges. digital transmission has emerged as the technique of choice due to its robustness and more efficient power and bandwidth utilization [3]. [4].

Bandwidth limitations have motivated considerable investigation into Continuous Phase Modulation (CPM) techniques [5]. [6]. [7]. CPM signals are of interest due to the spectral efficiency which they can achieve. coupled with their constant envelope property. These properties are particulariy atractive for mobile communications systems such as terrestrial cellular radio which are interference limited (demanding stringent adjacent channel interference specifications) while being cost driven since non-linear amplifiers may be used without producing spectral regrowth. Gaussian MSK [S]. a particular instance of CPM. has been chosen for the European cellular radio system (GSM).

Bandwidth limitations have also motivated the trend towards the use of higher frequency bands. While current mobile communications systems operate at frequencies at L-band (1-2 GHz) or below, systems urilizing spectrum at up to K3-band (27-40 GHz) are being investigared [9]. A significant amount of spectrum is allocated for mobile communications services in these higher frequency bands. 
Commensurate with the higher frequencies are higher fading rates. Fading rates are proportional to both vehicle speed and carrier frequency: therefore. future systems could experience fading rates one or two orders of magnitude higher than those found at L-band. For example. a vehicle travelling at $100 \mathrm{~km} / \mathrm{hr}$. and receiving at a carrier frequency of $1550 \mathrm{MHz}$ with an omnidirectional antenna will experience a single-sided fading bandwidth of approximately $144 \mathrm{~Hz}$. For a signal transmitted at $R=\$ \$ 00$ symbols/second. this represents a fading bandwidth of $0.03 R$. At $15.5 \mathrm{GHz}$, the single sided fading bandwidth for the same scenario increases to $1435 \mathrm{~Hz}$. or $0.3 R$. This high fading rate is used as an upper bound in this thesis. representing an approximation to the highest fading rate at which it still may be desirable to utilize a narrowband signal. At higher rates, other techniques such as a higher symbol rate TDMA may be more appropriate.

Terrestrial mobile communications channeis are often modelled as Rayleigh flat-fading channels [10]. Detection of signals on such channels has traditionally been performed in a noncoherent. or differentially coherent manner. The techniques utilized exhibit irreducible error floors at high fade rates. generate suboptimal soft decisions leading to power inefficiency and/or require large bandwidth. New power and bandwidth efficient techniques are required which overcome thesc problems for channels exhibiting high fade rates and flat-fading.

\subsection{Motivation For Investigating The MLAP Algorithm}

\subsubsection{Soft Decision Output}

The value of soft decision decoding of error correcting codes has long been recognized. In the past. soft decision decoding has generally been used at the front end of a receiver. with subsequent stages utilizing. and producing. hard decisions. This approach has produced near theoretical results for some systems where simple detectors/demodulators feed a single decoder. The transmission of BPSK over an AWGN channel is one such example. dot product detection of differential BPSK is another. One receiver structure for systems of this type is illustrated in Figure 1-1. 


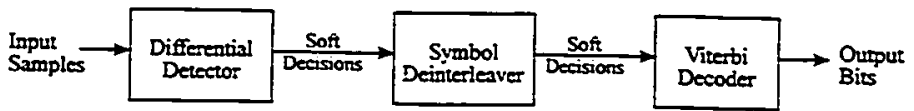

Figure 1-1 A traditional demodulator structure.

Today, digital transmission systems are moving towards more complex nested structures (see Figure 1-2). New transmission system designs often include one or more of the following:

- source encoders/decoders:

- multiple (inner/outer) forward error correction (FEC) codes. often with interleaving to reduce error bursts:

- trellis coded modulation (TCM):

- higher order constellations:

- channel equalizers.

All of the above techniques contribute to an increase in the number of states which would be required to perform optimal demodulation/decoding in a single stage. Because the

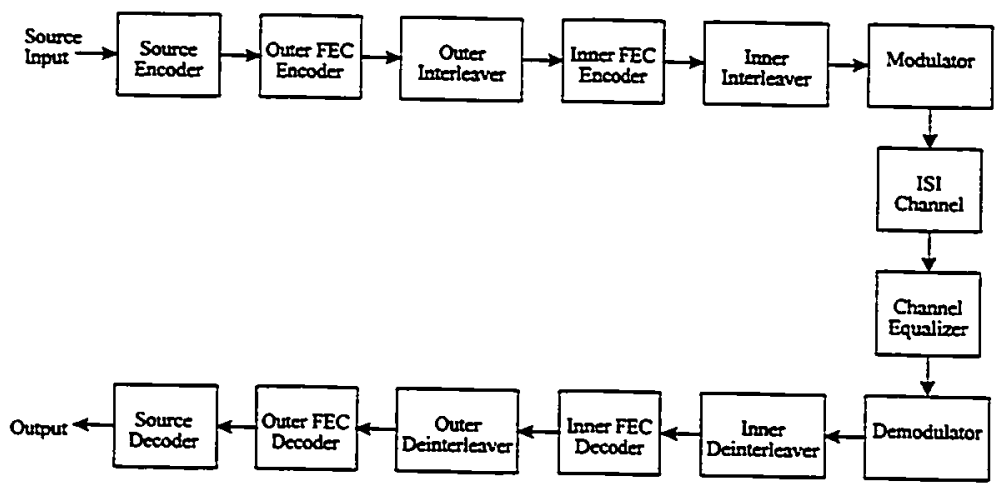

Figure 1-2 An example of a system utilizing concarenated processing techniques. 
number of states and thus the complexity of a decoder increases exponentially with the memory of the system. it is desirable to separate the demodulation/decoding process into a number of stages, each with sofi decision inputs and outputs. The goal is that a concatenation of stages producing near-optimal soft decisions will give performance which approaches that of a single stage with a large number of states. This goal is consistent with the principle stared by Viterbi [s].

"Never discard information prematurely that may be useful in making a decision until after all decisions related to that information have been completed."

Conventional soft decision decoding techniques generally produce soft decisions via some form of coherent. pseudo-coherent or differential detection. These soft decisions are often suboptimal. For partial response modulation schemes. the Viterbi algorithm is often used to make use of the redundancy introduced by the extended pulse shape. While this latter implementation has generally produced hard decisions, recent innovations such as the Soft Output Viterbi Algorithm (SOVA) [11]. allow soft decisions to be produced as well. It is of interest to determine how optimal these soft decisions are.

As is well known. the symbol-by-symbol MAP algorithm is the optimum decoding algorithm for codes that can be represented by a trellis of finite duration [12]. [13]. [14]. The algorithm is of interest because it produces, by definition. optimal soft decisions (decisions which have the maximum a posteriori probability). This thesis examines the use of the algorithm as a demodulation scheme rather than as a decoding scheme. As will be illustrated. the two applications are in fact very similar except that the output of the demotulation application is symbol soft decisions.

\subsubsection{Iterative Processing}

Recent work by Lodge et al. [15]. [16]. has shown that substantial coding gains may be achieved via iterative MAP processing techniques. The applications investigated to-date involve MAP filtering. a process whereby the outputs of multiple stages of MAP processing (which consist of probabilities for each code symbol) are fed back as augmented $a$ priori information. Subsequent iterations use the augmented $a$ priori probabilities to refine the decoding process. While this technique cannot reduce the 
overall probability of symbol error (by definition, a MAP decoder produces the minimum probability of symbol error), it can reduce the probability of word error by causing the decoder to converge to a valid codeword. Moher has shown that the iterative MAP processing approach follow's from entropy optimization principles [17].

A general representation of the MAP filtering process is illustrated in Figure 1-3. The processing at each stage refines the probabilities input to it. When the processing is iterated. the refined probabilities at the output of the $D$ th stage must be related back to the probabilities required at the input. Note that during the second and subsequent passes, the probabilities input to the ith stage must be processed to remove the refinements introduced by that stage in the previous pass.

Now consider the system of Figure 1-4 which is used to receive bits which have been coded. interleaved and transmitted over a channel with memory. Here, we replace the conventional demodulator with a MAP demodulator. On the first iteration. the a priori probabilities of all of the information bits are $1 / 2$. The probabilities determined by tive MAP demodulator are fed through the deinterleaver and then into a MAP filter for $a$ convolutional code. Utilizing the coding gain of the error correcting code, the MAP filter pruduces new code symbol probabilities which are then re-interleaved and processed to produce a new set of a priori probabilities for use by the MAP demodularor. In the final pass. thie code symbol probabilities output by the convolutional code filter are decoded to

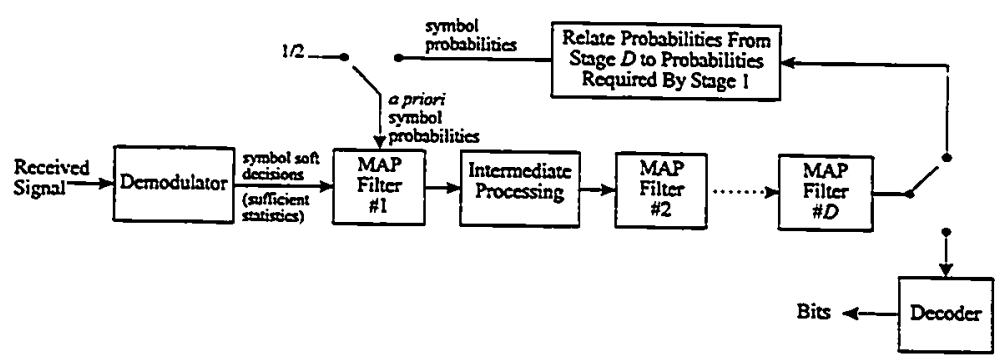

Figure 1-3 A receiver structure utilizing iterative MAP decoding. 


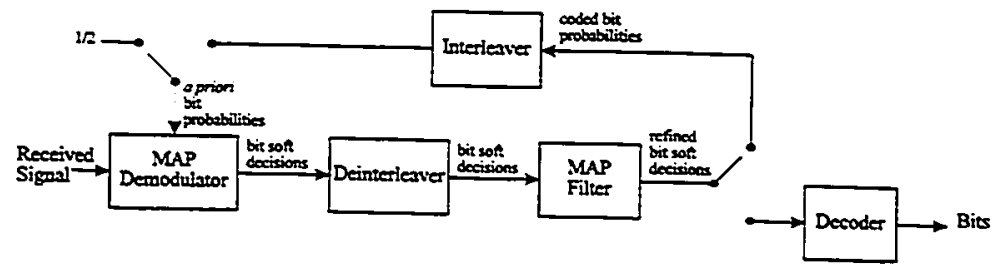

Figure 1-4 A receiver structure utilizing iterative MAP demodulation/decoding.

produce the recovered information bits.

The above system demonstrates a combined approach to demodulation and decoding. It is of interest to determine whether the same types of gains described in [15] for the decoding of product codes can be achieved for the case of combined demodulation and decoding. Any gains achieved should be applicable to any channel for which carrier recovery is difficult, whether it is due to high fading rates as considered herein, other impaiments such as phase noise, or simply a low operating $E_{5} / N_{0}$ due to the presence of powerful channel coding.

Note that iterative MAP demodulation alone cannor be expected to improve the soft decisions output from the demodulator since there is no coding gain to take advantage of (no codeword to converge to, and already optimal symbol-by-symbol decisions).

\subsubsection{BER Estimation}

In many applications, such as channel performance monitoring for power control. it is desirable to obtain an estimate of the raw bit error rate of a channel. Because the symbolby-symbol MAP algorithm inherently produces reliability estimates for each symbol. it is a simple matter to generate a raw BER estimate by transforming the symbol reliability estimates into bit reliability estimates. 


\subsection{Objectives Of Work}

This thesis has the following goals:

- to derive the symbol-by-symbol MAP algorithm for the demodulation of CPM signals on Rayleigh flat-fading channels:

- to determine the performance of the symbol-by-symbol MAP algorithm relative to that of alternate detection schemes:

- to evaluate the sensitivity of the algorithm to reduced state implementation or errors in the channel model;

- for cases where the computational complexity precludes the use of the MAP algorithm, to determine a reference for soft decision performance against which suboptimal detection schemes can be compared:

- to evaluate the use of the symbol-by-symbol MAP algorithm as an uncoded BER estimator, and.

- to evaluate the use of iterative MAP processing techniques for joint demodulation and decoding.

To accomplish these goals, a combination of theory, analysis and Monte Carlo simulation is used. First, the symbol-by-symbol MAP algorithm for the demodulation of CPM

signals on Rayleigh flat-fading channels is derived. Next. Monte Carlo simulation is used to evaluate the performance of the algorithm for a binary CPM signal when the fading spectrum and Gaussian noise level $\left(N_{0}\right)$ are known (the model is perfectly matched to the channel). This performance is then compared with that obtained with approximations to the coimposite fading plus noise spectrum. Performance with QPSK signals is also investigated.' The performance measures used to evaluate the algorithm include:

- hard decision bit error rate:

- bit error rate after decoding of a rate-1/2 convolutional code (used to evaluate the effectiveness of the soft decision outputs):

- average mutual information of hard and soft decisions; and,

\footnotetext{
' QPSK is considered to be a diserete time form of CPM.
} 
- computed raw bit error rate versus measured raw bit error rate.

The sensitivity of the algorithm to errors in the model and to the number of states is analyzed, and the results confirmed via simulation. Simulation techniques are also used to evaluate the effectiveness of joint MAP demodulation and decoding.

\subsection{Organization Of Thesis}

Chapter 2 provides background information useful for understanding the remainder of the work. Rayleigh fading channels, CPM and linear prediction techniques are reviewed and the use of the MAP algorithm for decoding of Markov processes is introduced.

Chapter 3 describes some techniques which are currently being used. or have been proposed. for demodulation of phase modulation in fast flat-fading channels. Particular emphasis is placed on differential detection and MLSE detection as benchmarks against which MAP detection will be compred.

In Chapter 4, the precise problem addressed by the thesis is defined. leading to Chapter 5 where the symbol-by-symbol MAP algorithm for the demodulation of CPM signals in Rayleigh flat-fading channels is derived. The result forms the basis for the implementation of the simulation.

Chapter 6 focuses on the performance of the MAP technique when used with binary CPM or QPSK signals. It is shown that the coded and uncoded BER performance of the symbol-by-symbol MAP demodulator remains good over a wide range of channel conditions. Chapter 7 investigates the use of iterative MAP processing (joint demodulation/decoding) to improve performance. It is shown that the performance of the iterative MAP receiver can approach that of a receiver utilizing ideal channel state information! Chapter 8 concludes with a summary of the results of the work, and suggestions for future research. 


\section{Chapter 2}

\section{Background}

This section introduces background information which is utilized in subsequent sections. While it is expected that the reader is already familiar with the concepts of fading channels. phase modulation and linear prediction, this section summarizes the aspects of these areas which are most relevant. and introduces notation which will be utilized throughout. MAP decoding of Markov processes is introduced in more detail.

\subsection{Rayleigh Fading Channels}

Extensive measurements have shown that the amplitude component of the fading on terrestrial mobile radio channel is characterized by a Rayleigh distribution. This distribution arises from the nature of the waves arriving at the mobile.

For terrestrial mobile radio communications. we make the assumption that there is no line-of-sight component to the received signal: instead. the received signal is made up of a number of reflections of the transmitted signal. These reflections arrive at the mobile via different paths and are therefore received with different amplitudes (due to the scattering properties of the reflectors) and phases (due to the delays introduced by each path). The amplitude of the received signal depends upon whether the recejved waves add constructively or destructively.

At baseband. it can be shown that the in-phase $(I)$ and quadrature $(Q)$ components of the signal vary independently. When the number of parhs is large ( $>7)$, the Central Limit Theorem asserts that the amplitude of each component has a Gaussian distribution [18]. 
By definition. this leads to a Rayleigh distribution of the received signal level. Deep fades of the signal envelope result when both $I$ and $Q$ fade simultaneously.

A model which is commonly used to represent the Rayleigh fading channel (with additive white gaussian noise) is shown in. Figure 2-1. Note that the effect of the fading on the signal $s(\tau)$ is entirely multiplicative.

In addition to the amplitude fading described above. the independent random variation of $I$ and $Q$ also leads to random variation in the phase of the received signal. The amount of variation is proportional to the depth of the fade. This phenomenon is termed random FM, and is responsible for the irreducible error floor exhibited by many detection schemes in the presence of fading since the phase variation is a multiplicative effect which does not decrease with increased SNR.

In addition to a random phase, each wave reaching the mobile has an associated Doppler shift which is a function of the speed of the vehicle and the angle between the direction of travel of the vehicle, and the incident wave. The distribution of the received Doppler shifts gives rise to an apparent widening of the received signal spectrum around the carrier frequency. The spectrum which would be observed for a CW signal is commonly referred to as the fading spectrum. Both its bandwidth and its general shape are

important. In digital transmission, the fading rate of interest is the ratio of the maximum

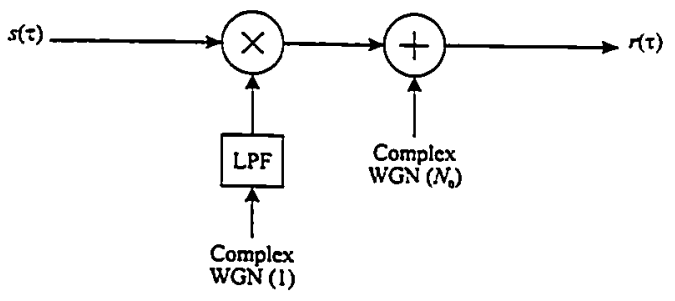

Figure 2-1 A block diagram of the model for a Rayleigh fading channel with additive white Gaussian noise (AWGN). WGN( $\sigma$ ) represents a white Gaussian noise process with variance $\sigma$. 
Doppler shift (i.e., the width of the fading spectrum) to the symbol rate.

There are a number of other parameters which complete the description of a fading channel: these are the delay spread and the coherence bandwidth. The delay spread is the time interval over which the majority of the signal energy due to a transmission at a given time is received while the coherence bandwidth describes the bandwidth over which the effects of the fading remain strongly correlated. Different bandwidths may apply when considering amplitude and phase correlations. The delay spread is inversely related to the coherence bandwidth, although its effect depends upon the distribution of the energy over the interval, in particular, whether the spread is generally uniform or whether it is made up of a number of diserete paths.

A flat-fading channel is one where the coherence bandwidth is much larger than the bandwidth of the signal of interest. This is the scenario considered in this thesis, and is often the case for narrowband signals.

\subsection{Continuous Phase Modulation (CPM)}

Continuous phase modulation (CPM) refers to a class of modulation schemes characterized by the restrictions that the envelope of the signal is constant and the phase of the signal always remains continuous (as compared with conventional PSK modulations where the phase of the signal may be discontinuous from one symbol interval to the next). The phase continuity constraint ensures an asymptotic rolloff of the power spectrum that is proportional to $l|| f-\left.f_{c}\right|^{4}$ (or better) since it eliminates sudden phase changes which introduce higher frequency components into the transmitted signa] spectrum [19].

A broad class of CPM signals can be represented as

$$
s(\tau)=\sqrt{\frac{2 E_{x}}{T}} \cos \left(2 \pi f_{c} \tau+\phi(\tau \cdot \mathbf{u}) \div \varphi_{0}\right)
$$

where the $E_{s}$ is the energy per symbol, $T$ is the symbol duration, $f_{c}$ is the carrier frequency, $\phi_{0}$ is the initial phase. $\phi(\tau u)$ is the time varying phase of the signal and $u$ is a vector of 
$Q$-ary information symbols. The information symbols are assumed to take on the values

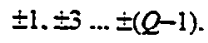

We are interested in the complex baseband (low-pass) representation

$$
s_{B}(\tau)=\sqrt{\frac{2 E_{r}}{T}} \exp [j \varphi(\tau . u)]
$$

where the information bearing excess phase function is

$$
\varphi(\tau, u)=2 \pi h \int_{-} \sum_{n=-\infty}^{\infty} u_{n} g(t-n T) d t
$$

with $h$ representing the modulation index and $g(\tau)$ describing the instantaneous frequency pulse shape. In practice. $g(\tau)$ is nonzero only over some finite period $v T$ where $v$ is a positive integer representing the length of the pulse in symbol periods. For a causal system,

$$
g(\tau)\left\{\begin{array}{ll}
=0 & \tau<0 . \tau>v T \\
\neq 0 & 0 \leq \tau \leq v T
\end{array} .\right.
$$

The conventional definition of the modulation index $h$ assumes that

$$
\int_{0}^{v r} g(\tau) d \tau=\frac{1}{2}
$$

From (2.3) and (2.4), the maximum phase change over any symbol period is then $\pi h(Q-1)$.

An infinite variety of CPM schemes can be created by varying the pulse shape, modulation index. and symbol alphabet. Note that in addition to the inherent memory introduced by the phase continuity, additional memory may be introduced by choosing a pulse shape which spans more than one symbol period. CPM schemes utilizing pulse shapes spanning a single symbol period are referred to as full response schemes while those utilizing pulse shapes which span several symbol periods are referred to as partial response schemes. If fully exploited, partial response schemes have the advantage of improving the spectral efficiency of the signal without, on AWGN channels, 
compromising performance. This may not be the case on fading channels where the channel state can change significantly over the duration of the partial response symbol.

If the modulation index $h$ is chosen to be rational. the resulting CPM signal can be described using a finite number of states, and can therefore be represented as a trellis. For this type of CPM signal transmitted over an AWGN channel. the optimum coherent detector is a maximum likelihood sequence estimator (MLSE), generally implemented via the Viterbi algorithm.

\subsection{Linear Prediction}

Linear prediction deals with the problem of developing a model for the behaviour of a signal. The model of interest describes a system whose output at a given time can be expressed as a linear combination of its past outputs. and its past and present inputs.

Mathematically, this can be expressed as

$$
s(n)=-\sum_{l=1}^{L} a_{l} s(n-l)+G \sum_{j=0}^{l} b_{j} u(n-j)
$$

where $n$ is the discrete time index. $s(n)$ is the output at time $n . u(n)$ is the input at time $n$ and $b_{0}=1$. The parameters of the system are the $\left\{a_{l}\right\} .\left\{b_{j}\right\}$ and $G[20]$.

When viewed in the frequency domain, the model becomes

$$
H(z)=\frac{S(z)}{U(z)}=G \frac{1+\sum_{j=1}^{j} b_{j} z^{-j}}{1+\sum_{l=1}^{L} a_{i} z^{-l}}
$$

where $S(z)$ and $U(z)$ are the $z$-transforms of $s(n)$ and $u(n)$ respectively. The equation in (2.6) presents a pole-zero model of the system, with the numerator term representing the $J$ zeroes. and the denominator term the $L$ poles.

In this thesis. we are interested in the all-pole case, i.e.. the case where all the $\left\{b_{j}\right\}(j>0)$. are zero. This is the most commonly utilized linear prediction model. and a substantial amount has been written about its applications and methods of estimating its parameters [20]. In the all-pole case. (2.5) and (2.6) simplify to 


$$
s(n)=-\sum_{i=1}^{2} a_{t} s(n-l) \div G u(n)
$$

and

$$
H(z)=\frac{S(z)}{U(z)}=\frac{G}{1+\sum_{i=1}^{L} a_{1} z^{-l}}
$$

respectively. One means of estimating the predictor coefficients $\left\{a_{l}\right\}$ for a random signal is the method of least squares. With this method, we minimize the mean squared error between the actial value of $s(n)$, and the value. $\hat{s}(n)$, predicted from a linear combination of past samples, i.e.. minimize

$$
E\left[(s(n)-\hat{s}(n))^{2}\right]=E\left[\left(s(n)+\sum_{l=l}^{L} a_{i} s(n-l)\right)^{2}\right]
$$

For a zero mean stationary process, minimizing (2.9) reduces to solving the $L$ equations in $L$ unknowns given by

$$
\sum_{i=1}^{L} a_{l} r_{i-l}=-r_{i}
$$

where $1 \leq i \leq L$ and $r_{i}$ is the autocorrelation function

$$
r_{i}=E[s(n) s(n+i)]
$$

which can be estimated from a time average if the process is ergodic. Because of their special form, the equations in (2.10) can be solved efficiently using Durbin's recursive proceciure [20]. A number of points should be noted:

- the solution is unchanged if normalized autocorrelation coefficients are used. i.e..

$$
\rho_{i}=\frac{r_{i}}{r_{0}}:
$$

- the minimum total error decreases. or remains the same. as the order of the predictor, $i$. increases, i.e.,

$$
0 \leq \varepsilon_{i} \leq \varepsilon_{i-1}, \quad \varepsilon_{0}=r_{0}:
$$


- the predictor coefficients can be expressed as the $\left\{a_{t}\right\}$ described above. or as a set of inte:mediate parameters of the algorithm known as reflection coefficients $\left\{k_{l}\right\}$ :

- an exfected nomalized prediction ersor. i.e..

$$
V_{1}=\frac{\varepsilon_{1}}{r_{0}}
$$

for a $L$ th order predictor can be computed from the reflection coefficients using

$$
V_{L}=\prod_{i=1}^{L}\left(1-k_{i}\right)^{2}
$$

- the expected prediction error can be used to choose the predictor order which represents the best tradeoff between complexity and performance.

In summary. linear prediction methods provide a means of characterizing the output of a system (whose second order model is known. but whose excitarion is unknown) using samples of its past outputs. The application of interest in this thesis is the estimation of the fading process (which, as described in Section 2.1. can be modelled as a linear filier excited by a white gaussian noise source).

\subsection{MAP Decoding Of Markov Processes}

The symbol-by-symbol MAP algorithm (hereinafter referred to simply as the MAP algorithm) was introduced by Bahl et al. in [12] as an efficient way of optimally decoding linear block and convolutional codes transmitted through a discrete memoryless channel (DMC). In fact. its use extends to any source which can be modelled as a discrete-ime finite-state Markov process (i.e., by a trellis of finite duration).

The MAP algorithm is a solution to the following problem:

Given a sequence of observations. Y. of a discrete-time finite-state Markov process in memoryless noise. find the sequence $\hat{U}$ which maximizes the a posteriori probability of the detected symbols. i.e., maximizes $\operatorname{Pr}\left\{\hat{u}_{i} \mid Y\right\}$ for all $i$. where $\hat{u}_{i}$ is the ith element of $\hat{\mathbf{U}}$. 
Unlike the Viterbi algorithm which minimizes the probability of a word error. the MAP aigorithm minimizes the probability of symbol error. A consequence of this is that the chosen sequence $\hat{U}$ may or may not be a valid codeword.

The MAP algorithm can be best understood via an example. Consider the trellis shown in Figure 2-2a where the nodes correspond to states and the branches represent possible transitions. Assume that we wish to compute the probability that the system is in state 0 at time $t=2$. If this probability is to be evaluated based on the entire received sequence. the quantity of interest is $\operatorname{Pr}\left(S_{z}=0 \mid Y_{0}^{*}\right)$. where superscripts and subscripts are time indices with $Y_{0}^{5}$ representing the sequence of received samples from $t=0$ to $t=5$.

One way of computing this probability would be to sum the probabilities. conditioned on $Y_{0}^{5}$. of all of the paths through the trellis which pass through state 0 at time $t=2$. The branches making up these paths are shown as solid lines in Figure 2-2b with the corresponding nodes listed in Table 2-1. The shaded columns in the table represent

\begin{tabular}{|c|c|c|c|c|c|c|}
\hline \multicolumn{1}{c|}{} & \multicolumn{7}{|c|}{ Time $(t)$} \\
\cline { 2 - 7 } Path & 0 & 1 & 2 & 3 & 4 & 5 \\
\hline 1 & 0 & 0 & 0 & 0 & 0 & 0 \\
\hline 2 & 0 & 0 & 0 & 0 & 1 & 0 \\
\hline 3 & 0 & 0 & 0 & 1 & 0 & 0 \\
\hline 4 & 0 & 0 & 0 & 1 & 2 & 0 \\
\hline 5 & 0 & 1 & 0 & 0 & 0 & 0 \\
\hline 6 & 0 & 1 & 0 & 0 & 1 & 0 \\
\hline 7 & 0 & 1 & 0 & 1 & 0 & 0 \\
\hline 8 & 0 & 1 & 0 & 1 & 2 & 0 \\
\hline
\end{tabular}

Table 2-1 The states traversed by paths passing through state 0 at time $t=2$. 
parameters which are fixed, i.e., the initial state. the final stare, and the stare of interest. The remaining columns represent degrees of freedom. For the simple trellis of Figure 2-2, there are only eight possible paths: however, for a practical trellis there will be considerably more since the number of paths grows exponentially with $t$.

The seale of the problem is reduced if the node probability of interest is looked at in terms of the node and branch probabilities forward and backsward one time interval. For the current example. this means examining the conditional node probabilities at $t=1$ and $t=3$. and the conditional branch probabilities from $t=1$ to $t=2$ and $t=2$ to $t=3$.

Figure 2-2c shows the relevant probabilities (joint probabilities are shown because these are the values computed by the algorithm: conditional probabilities are easily obtained from these). Uising the fact that events after time $t$ depend only on the state of the process at time $t$, we can write:

$\bar{\lambda}_{z}(0)=\operatorname{Pr}\left(S_{z}=0: Y_{1}^{5}\right)$

$=\operatorname{Pr}\left(S_{z}=0 ; Y_{1}^{2}\right) \cdot \operatorname{Pr}\left(Y_{3}^{5} \mid S_{2}=0\right)$

$=\sum_{m=0}^{N-1} \operatorname{Pr}\left(S_{1}=m^{\prime}: Y_{1}\right) \cdot \operatorname{Pr}\left(S_{z}=0: Y_{2} \mid S_{1}=m^{\prime}\right) \cdot \sum_{m=0}^{M-1} \operatorname{Pr}\left(S_{3}=m: Y_{3} \mid S_{2}=0\right) \cdot \operatorname{Pr}\left(Y_{s}^{s} \mid S_{3}=m\right)$

where $\bar{\lambda}(m)$ is the joint probability that the system is in state $m$ at time $t$ and the received signal. and $M$ is the total number of states in the trellis. Depending upon the structure of the trellis, some of the node and branch probabilities will be zero.

Examining the expression above. we can see that its two pars correspond to information up to time $t=2$. and information from time $t=3$ onwards. If we write:

$$
\begin{aligned}
& \bar{\alpha}_{t}(m)=\operatorname{Pr}\left(S_{t}=m: Y_{1}^{2}\right) . \\
& \bar{\beta}_{z}(m)=\operatorname{Pr}\left(Y_{t=1}^{S} \mid S_{t}=m\right)
\end{aligned}
$$

and

$$
\tilde{\gamma}_{t}\left(m^{\prime} \cdot m\right)=\operatorname{Pr}\left(S_{t}=m: Y, \mid S_{t-1}=m^{\prime}\right)
$$




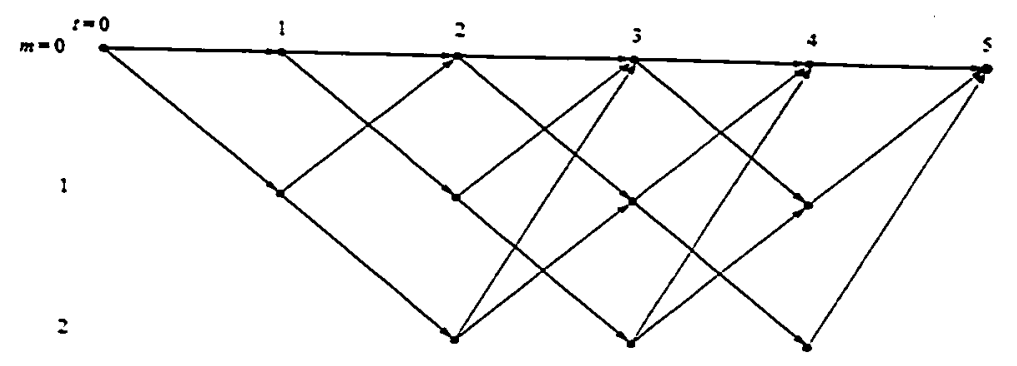

(a)

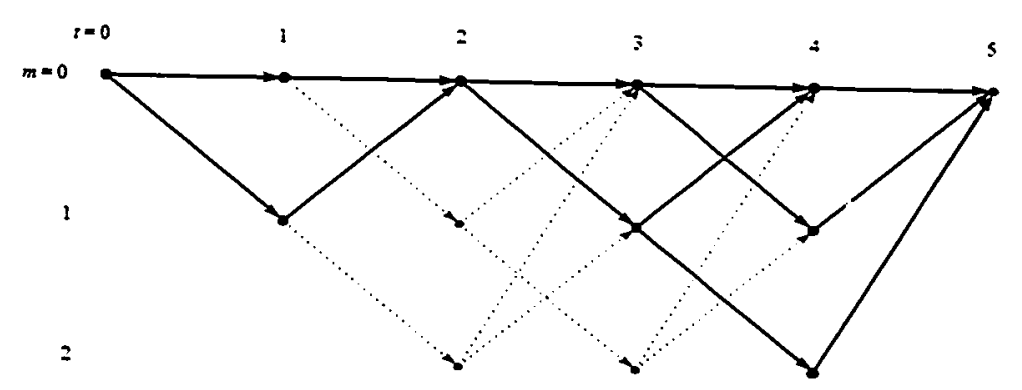

(b)

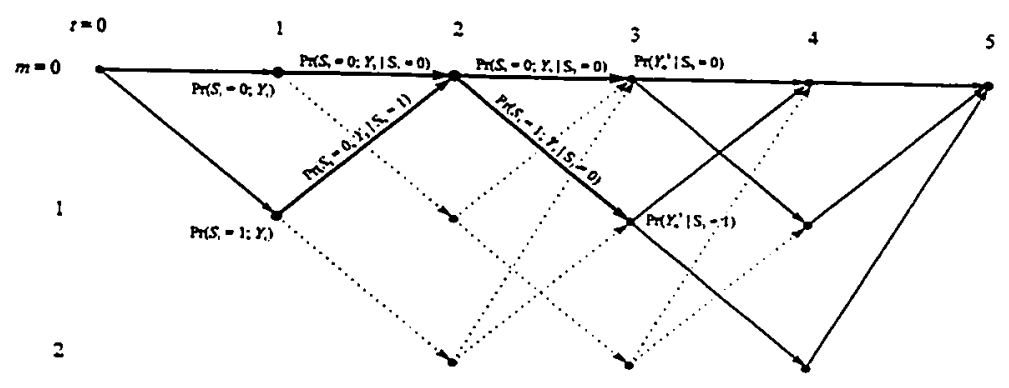

(c)

Figure 2-2 The trellis of an example Markov process. 
then we get

$$
\begin{aligned}
\tilde{z}_{2}(0) & =\tilde{\alpha}_{2}(0) \cdot \tilde{\beta}_{2}(0) \\
& =\sum_{m^{\prime}=0}^{N-1} \tilde{\alpha}_{1}\left(m^{\prime}\right) \cdot \tilde{\gamma}_{2}\left(m^{\prime}, 0\right) \cdot \sum_{m=0}^{N-1} \tilde{\gamma}_{3}(0, m) \cdot \tilde{\beta}_{3}(m) .
\end{aligned}
$$

Thus

$$
\bar{\alpha}_{t}(m)=\sum_{m^{\prime}=0}^{M-1} \bar{\alpha}_{t-1}\left(m^{\prime}\right) \cdot \bar{\gamma}_{t}\left(m^{\prime} \cdot m\right)
$$

and

$$
\tilde{\beta}_{t}(m)=\sum_{m^{\prime}=0}^{M-1} \tilde{\gamma}_{t+1}\left(m, m^{\prime}\right) \cdot \tilde{\beta}_{i+1}\left(m^{\prime}\right)
$$

The derivation above demonstrates that the state probability can be expressed as a product of two terms, each of which can be computed recursively. It is this recursive structure that allows the MAP algorithm to efficiently compute all of the node and branch probabilities in the trellis. If branch probabilities are of interest, then for a sequence of length $N$, they can be expressed as

$$
\begin{aligned}
\tilde{\sigma}_{t}\left(m^{\prime}, m\right) & =\operatorname{Pr}\left(S_{t-1}=m^{\prime}: S_{t}=m: X_{0}^{N-1}\right) \\
& =\tilde{\alpha}_{i-1}\left(m^{\prime}\right) \cdot \tilde{\gamma}_{t}\left(m^{\prime}, m\right) \cdot \tilde{\beta}_{t}(m)
\end{aligned} .
$$

Once all of the node and branch probabilities are known. the symbol probabilities at each time $t$ can be ccimputed as shown in [12]. 


\section{Chapter 3}

\section{Current Techniques For Demodulation In Flat Fast Fading Channels}

This section describes some of the techniques which are currently used. or have been proposed, for the demodulation of PSK or CPM signals in fast fading channels. Here. fast fading is defined as meaning that the phase of the fading process can vary significantly over a symbol period: i.e.. where the symbol duration is a significant fraction of the coherence time of the channel [21, p.715]. Traditionally, fast fading has been used to describe channels where the fade rate is at least $1 \%$ of the symbol rate [22]. although in this thesis we wish to examine channels where it is significantly higher.

Demodulation techniques can be divided into three general categories - ideal coherent. pseudo-coherent, and non-coherent detection. Of these methods, coherent detection is generally impractical in fast fading channels. The use of non-coberent methods on fast fading channels has received considerable attention, but these techniques all suffer considerable performance penalties when compared to ideal coherent detection. In this thesis, emphasis is placed on pseudo-coherent methods where some use is made of carrier phase information. In Chapter 6. differential detection and MLSE detection will be used as benchmarks against which the performance of symbol-by-symbol MAP detection will be compared.

\subsection{Ideal Coherent Detection}

In order to perform coherent detection of phase modulated signals, the phase of the transmitted carrier must be recovered. Conventional carrier recovery techniques utilize a 
combination of a non-linearity and a phase-locked loop to extract the carrier phase. The difficulty with this approach on a fast fading channel is that the requirement for narrowing the loop filter to reject noise conflicts with the requirement to widen the filter to track the fading process. This conflict is inherent and renders this approach unfeasible on fast fading channels.

\subsection{Differential Detection}

Traditionally, the technique of choice for demodulation of PSK signals on severely faded channels has been differential detection. While various forms of differential detectors exist. the common property which makes them attractive for use on fading channels is their lack of reliance on an accurate carrier phase estimate such as is required for coherent derection. Additionally. standard differential detection is easy to implement and provides good performance under a variety of fading conditions (other than the inherent degradation in going to differential detection in AWGN).

For differential detection, the information is encoded into the phase difference between consecutive symbols (differential encoding). At the receiver, detection is performed by simply comparing the signal phase of a given received symbol with that of the previously received symbol. One receiver structure which implements this processing on a complex baseband signal is shown in Figure $3-1$.

Note that the receiver structure shown in Figure 3-1 is suboptimum since the reference for a decision in a given symbol interval is the received signal from the previous symbol interval. This signal is inherently quite noisy since unlike a coherent reference obtained as described in Section 3.1. the received signal cannot pass through a narrowband filter (this is of course the same property that makes differential detection useful on fast fading charinels). 


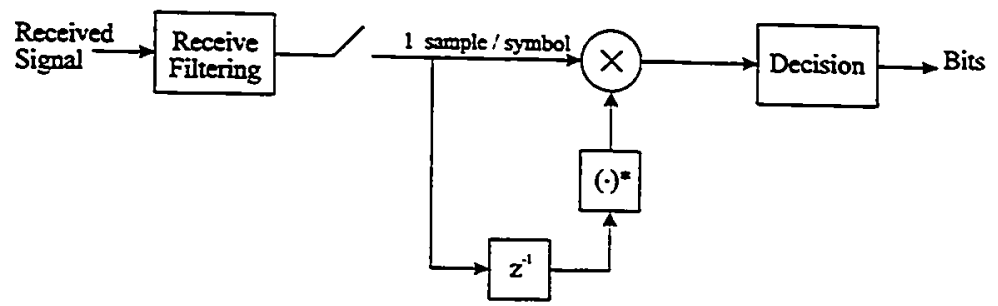

Figure 3-1 A simple differential detector.

Unformanately, while differential detection produces a good soft decision for low order signal constellations (e.g. BPSK. MSK. QPSK). it is not nearly as effective for higher order ones (e.g. 8-PSK) [23]. This can be seen in the BER results shown in Figure 3-2. where the difference in performance between differential 8-PSK relative to differential BPSK is far greater at the higher fading rate. Figure 3-2 also shows the irreducible error floor that is characteristic of differential detection on fading channels. This error floor is a result of the effect of random FM increasing with the fading rate. thus eroding the assumption of constant carrier phase over two successive symbol intervals.

It should also be noted that since differential detection only uses information spanning a single symbol period. it is not suitable for taking advantage of the redundancy present in spectrally efficient signals which utilize memory in the modulator. 


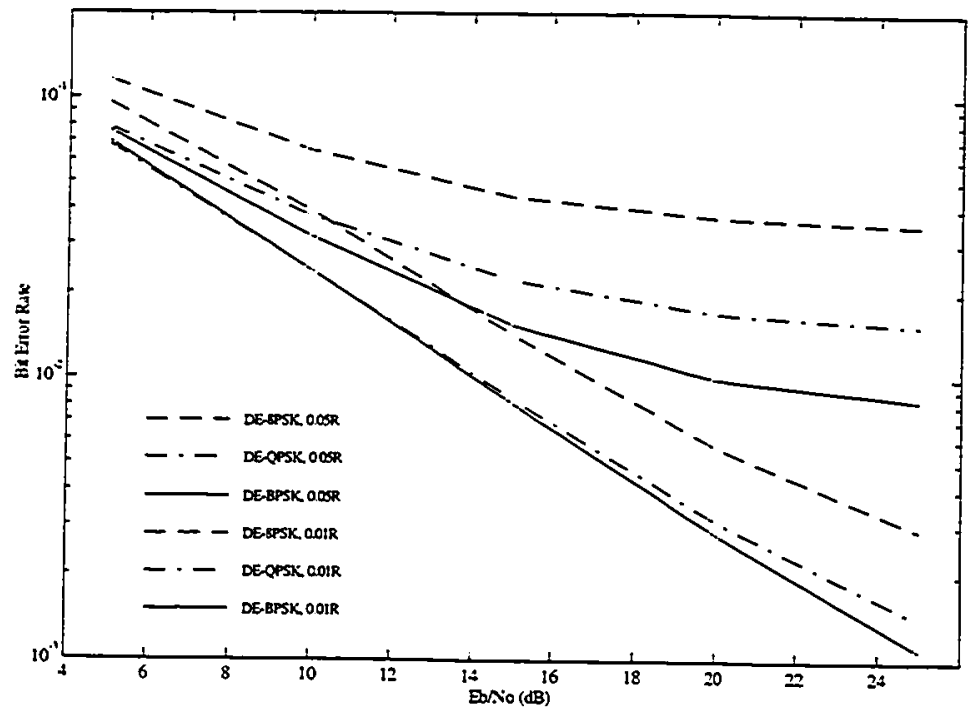

Figure 3-2 The BER performance of differential detection of differentially encoded BPSK. QPSK and 8-PSK at fade rates of $0.01 R$ and $0.05 R$.

\subsection{Multiple Symbol Differential Detection (MSDD)}

To try to reduce the difference between the performance of differential detection and the performance of coherently detected differentially encoded PSK. Divsalar and Simon suggested performing a maximum likelihood sequence estimation (MLSE) on the phase differences between a number of successive symbols. In [23], this multiple symbol differential detection (MSDD) scheme is derived for the detection of M-ary PSK on an AWGN channel. Performance gains from $0.5 \mathrm{~dB}$ (BPSK) up to $2 \mathrm{~dB}$ (for 8-PSK) were shown to be achievable by performing MISE over only a few symbol periods. As the observation interval increases towards infinity, the performance approaches that of ideal coherent detection of differentially encoded PSK. 
The operation of the MSDD algorithm is predicated on the assumption that the channel phase is essentially constant over the observation interval. This assumption breaks down on fading channels. particularly those where the fading rate becomes a significant fraction of a symbol period. In [24]. Divsalar and Simon examined the operation of the algorithm on fading channels. It was shown that for the Rayleigh fading case, MSDD produces no gain over conventional differential detection and may in fact introduce a loss.

Algorithms related to MSDD have been proposed by a number of authors [25]. [26]: however, these have been oriented towards improving computational efficiency rather than improving performance.

As with conventional differential detection, MSDD is normally used with differentially encoded PSK. An alternate approach is to periodically insert known reference symbols which can be used to resolve the phase ambiguity. As the fading raic increases, this latter approach requires an increasing number of reference symbols to ensure that phase slips are detected and corrected.

\subsection{Pilot Symbol Assisted Modulation (PSAM)}

As power efficiency has become more and more important, pilot symbol techniques have received increased attention [27], [28], [29], [30]. These techniques, known variously as pilot symbol assisted modulation (PSAM) or pilot symbol insertion (PSI). use known pilot symbols inserted into the transmitted data stream to assist the demodulator in determining the gain and phase characteristics of the channel. The pilot symbols thus provide a means for the demodulator to extract a pseudo-coherent reference. PSAM is the dual of earlier techniques such as transparent tone-in-band (TTIB) and tone calibration which use a pilot tone to provide the same reference [31]. [32]. 
Figure 3-3 shows a block diagram of a PSAM transmission system. At the transmitter, the input bits are mapped to symbols and then the resulting stream is multiplexed with a known sequence of pilot symbols. One pilot symbol is inserted for every $(P-1)$ data symbols. The resulting symbol stream is depicted in Figure $3-4$. The composite symbol stream passes through a puise shaping filter and is transmitted over the channel. At the receiver. matched filtering is performed to extract optimal symbol samples. and then the composite stream is demultiplexed back into the data and pilot streams. Channel estimation is then performed using the pilot stream. First the known modulation is stripped off of the pilot symbols. The resulting carrier is low-pass filtered. This filter is analogous to the loop filter in a phase-locked loop. except that being a feedforward system. it does not suffer from hang up. The bandwidth of the filter is matched to the maximum expected fading bandwidth. Once channel samples have been recovered. they

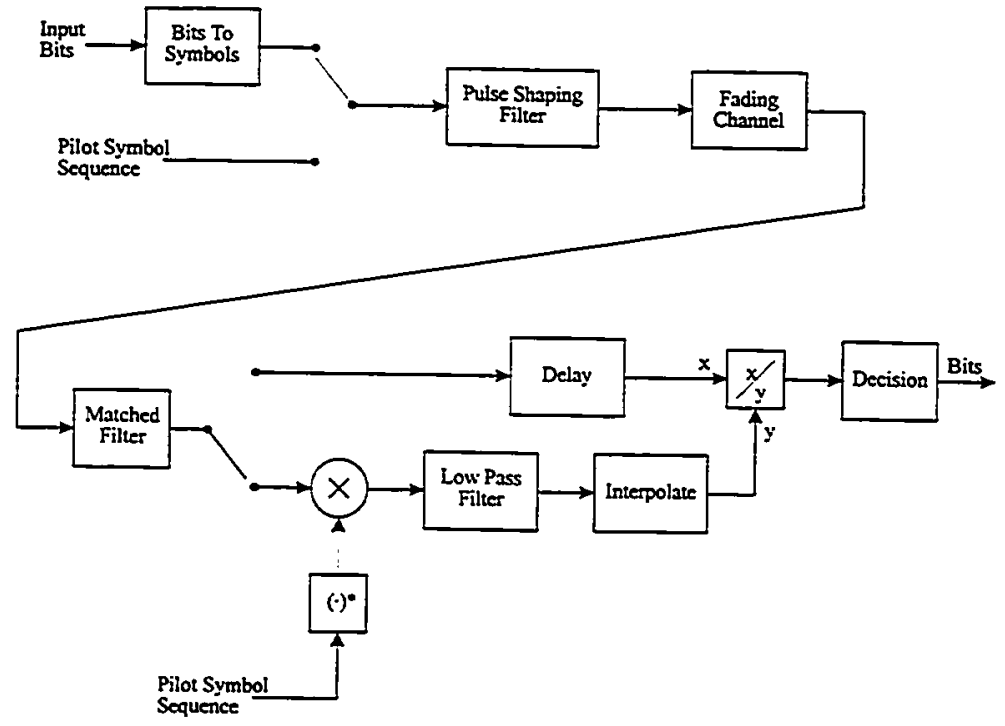

Figure 3-3 A transmission system utilizing pilot symbol assisted modulation. 


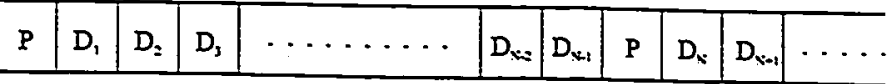

Figure 3-4 The PSAM transmitted symbol stream with one pilot symbol per $P-1$ data symbols.

are interpolated up to the data symbol rate. and used to apply the inverse of the channel response to the data symbols. This constitutes the pseudo-coherent detection.

The pilot symbol rare and the low pass filter bandwidth are the critical parameters in the design of a PSAM system. In the absence of noise and interference. and provided that the pilot symbol rate exceeds the minimum required to sample the expected fading bandwidth. the fade characteristies can, by Nyquist's sampling theorem. be estimated exactly. Liu and Feher [30] have shown that if the pilot symbol rate exceeds the minimum. the resulting performance approaches that of ideal coherent PSK rather than that of differential PSK. albeit with a corresponding penalty in bandwidth requirements. This performance improvement is obtained because the bandwidth of the low pass filter (which is a function of the pilot symbol spacing) can be reduced.

The advantage of PSAM when compared to pilot tone techniques is that PSAM introduces no changes in the transmitted pulse shape, or peak to mean power ratio. Furthermore, it is generally more bandwidth efficient [28] and requires less complex signal processing.

PSAM has a number of desirable properties; these include:

- independence of the channel state estimation mechanism from the modulation or channel coding (the pilot symbols need not be part of the transmitted signal constellation and need not be taken into account during coding and decoding);

- robustness against random FM effects. leading to a lowering of the irreducible error floor commonly observed at high fade rates:

- no requirement for differential encoding since the pilot symbols provide an unambiguous phase reference; 
- inherent compensation for residual frequency offset.

The major disadvantage of the PSAM approach is that the pilot symbol rate required to track the fading process is directly proportional to the fading bandwidth (by Nyquist's sampling theorem). Consequently, at high fade rates, the ratio of pilot symbols to data symbols is high, imposing a significant power and bandwidth penalty. In general, the pilot symbol spacing must be set based on the worst-case fading bandwidth: this degrades the performance of PSA.M relative to other techniques at times when the actual fading bandwidth is small because of the noise which passes through the pilot symbol filter. If the Doppler spread exceeds the bandwidth of the pilot symbol filter. a threshold effect can be observed (particularly for fading spectra such as the land mobile channel which have most of the energy in the fading process concentrated at the extremes).

\subsection{Linear Predictive (MLSE) Detection}

Traditional coherent. or pseudo-coherent. detection techniques perform some sort of carrier recovery. and then use the recovered carrier to perform coherent detection of the transmitted data. Since the transmitted data is the only information which is actually required. this two-step approach represents just one means of recovering the data. An alternative approach is to directly estimate the data from the received samples.

In [33]. Lodge and Moher develop a receiver structure for fading channels which uses maximum likelihood sequence estimation (MLSE) to determine the data sequence which produces the most likely channel samples. A general approach is described which utilizes the Viterbi algorithm in conjunction with Kalman filtering. A simplified implementation is then derived for the reception of CPM signals over Rayleigh flat-fading channels. Since this latter receiver structure forms the basis for the MAP demodulator described in this thesis. a detailed explanation is deferred until Chapter 5: however. a summary is given below.

\subsubsection{Approach}

Consider a communications system whose input is a $N$-vector $\mathrm{X}(k)$ which represents one of $\{\mathbf{X}(k)\}$ possible messages. If the corresponding received signal is a $N$-vector $\mathbf{X}$, then the goal of a maximum likelihood detector is to choose the hypothesis $k$ such that 
It is shown in [33] that (3.1) leads to a log-likelihood function which can be written in terms of a summation of the pdf of the received samples conditioned on the input. and on previously received samples. Using the fact that the received samples have a complex Gaussian distribution. a likelihood index is derived. The maximum likelihood hypothesis is then shown to be the hypothesis with the minimum likelihood index. i.e., the maximum likelihood hypothesis is $k$ if

$$
\Lambda_{N}^{k}(\mathbf{Y})<\Lambda_{N}^{i}(\mathbf{Y}) \text { for all } i=k .
$$

where $\Lambda_{N}^{k}(\mathrm{Y})$ represents the likelihood index for hypothesis $k$ of a length $N$ sequence. For the case of CPM signals transmitted over Rayleigh flat-fading channels, it is further shown that the likelihood index can be computed via an update of the form

$$
\Lambda_{j}^{k}(Y)=\Lambda_{j-1}^{k}(X)+\left|y_{j}^{\prime}(k)-y_{j-1}^{\prime}(k)\right|^{2}
$$

where $y_{j}^{\prime}(k)$ represents the $j$ th received sample after the hypothesized modulation has been removed and the update term represents the squared prediction error for the current sample. A dynamic programming algorithm (the Viterbi algorithm) is used to compute the likelihood indices and to choose the maximum likelihood hypothesis. Essentially, the resulting output sequence is that which. if used to remove the hypothesized modulation. produces the minimum channel prediction error.

\subsubsection{Receiver Structure}

The overall structure of the linear predictive receiver is shown in Figure 3-5. Received samples enter a bank of FIR filters, $r$ per hypothesis. where $r$ is the number of samples per symbol. The filters remove the appropriate hypothesized modulation. and then generate a prediction error. This error is squared and then input to the Viterbi algorithm which performs the maximum likelihood sequence estimation. The coefficients of the filters are dependent upon the hypothesized modulation and upon the composite fading plus noise power spectral density as approximated by a linear predictor. 


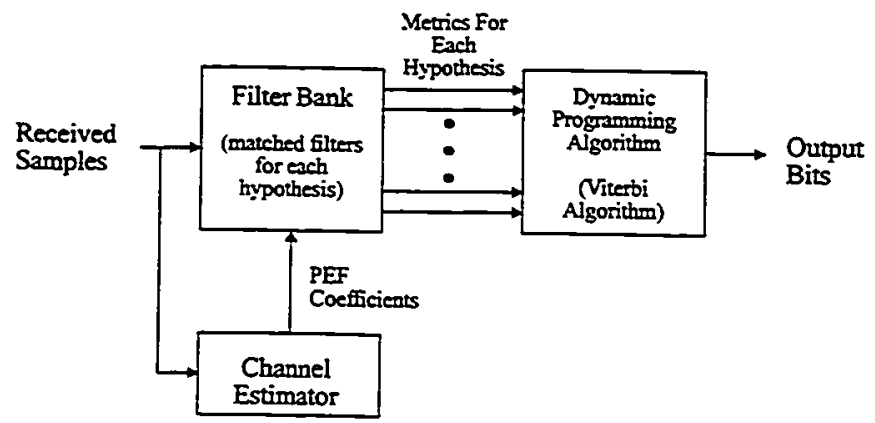

Figure 3-5 The structure of the Linear Predictive Receiver.

\subsubsection{Performance}

Simulations were performed on the linear predictive receiver for a binary CPM scheme utilizing the N32FM pulse shape [19]. For a single-sided fading bandwidth of 0.01 times the symbol rate $(0.01 R)$, the linear predictive receiver performed vily marginally better than standard differential detection: however, for a fading bandwidth of $0.3 R$. the differential detection scheme produced an irreducible error floor of approximately 0.3 (raw bit error rate) while the linear predictive receiver exhibited impressive performance. and no error floor. 


\section{Chapter 4}

\section{Problem Statement}

\subsection{Optimal Soft Decisions In Fast Fading}

This thesis examines the general problem of producing optimal soft decisions for digital signals received on fast fading channels. In particular, demodulation of CPM signals transmitted over Rayleigh flat-fading channels is investigated.

Fast fading channels are considered to be those where the phase of the fading process can vary significantly over a symbol period. In the terminology of Section 2.1. this corresponds to channels where the single-sided fading bandwidth is a significant proportion of the symbol rate. While the term fast fading has been used to describe channels where the fade rate is as low as $1 \%$ of the symbol rate. we wish to consider channels where it is much higher. In this thesis. fade rates as high as $30 \%$ of the symbol rate are used.

\subsection{Disadvantages Of Current Approaches}

While simple differential detection is the most common means of combating fading for phase modulated signals. it suffers from a large performance degradation as the fade rate increases. The degradaiion due to the fading is independent of the signal-to-noise ratio. introducing an irreducible error floor. Increased complexity multiple symbol differential detection techniques are no more effective because their core assumption of near-constant channel phase over a number of symbol periods breaks down at high fade rates.

Pilot symbol techniques overcome the error floor limitations of differential detection and are a good solution for low to moderate fade rates. Unfortunately, while the performance 
of pilot symbol techniques can be maintained at high fade rates. the extra power and bandwidth required becoi...s excessive. effectively reducing the information throughput. This is a clear consequence of the requirement to sample the channel faster than the Nyquis! rate corresponding to the fading bandwidth.

The MLSE-based linear predictive receiver overcomes both the error floor limitation of differential detection and the power/bandwidth limitation of pilot symbol approaches. Its major drawback is that it produces hard decisions via the Viterbi algorithm. This makes it unsuitable for applications requiring soft decisions in subsequent stages. Modifications to the Viterbi aigorithm, such as the Soft Output Viterbi Algorithm (SOVA) described in [11] may prove effective for binary modulation schemes.

\subsection{Advantages Of Symbol-By-Symbol MAP Demodulation}

The performance of the MLSE-based linear predictive receiver clearly indicates that significant gains can be achieved by utilizing the energy in the entire transmitted signal in conjunction with a statistical characterization of the channel. The use of the symbol-bysymbol MAP algorithm for demodulation. rather than decoding. presents a means of applying the same philosophy while obtaining optimal soft decisions. These soft decisions can then be passed to subsequent stages. allowing maximal gain to be obtained from each.

As was the case with the MLSE-based linear predictive receiver, symbol-by-symbol MAP demodulation requires no power/bandwidth expansion.

Another advantage of symbol-by-symbol MAP demodulation is that the soft decisions produced. in addition to being optimal, are in fact reliability estimates on the symbols in question. i.e.. symbol probabilities. These symbol reliability estimates can be used to compute a true bit error rate for the channel. They can also be used in conjunction with measured performance data to provide an accirate estimate of the channel noise level $\left(N_{0}\right)$. This estimate can then be fed to portions of the demodulator which may require it for optimal performance.

Since symbol-by-symbol M.AP demodulation can utilize a priori information about the transminted signal, the algorithm also affords the possibility of performing iterative 
processing. where refined inpur symbol probabilities are fed back to the demodulator as a priori information. This approach has demonstrated significant potential for decoding applications but has not yet been investigated for combined demodulation/decoding applications. 


\section{Chapter 5}

\section{Symbol-By-Symbol MAP Demodulation}

In this section, the symbol-by-symbol MAP algorithm is applied to the demodulation of CPM signals transmitted over Rayleigh flat-fading channels. This work is the first application of the MAP algorithm to this scenario.

\subsection{Approach}

Given a particular sequence of received samples, the goal of symbol-by-symbol MAP demodulation is to determine. for all possible symbols and all possible times $t$, the probability that a particular symbol was transmitted at that time. Once these probabilities have been determined. the demodulator can use them directly as symbol soft decisions, or can process them further to extract bit soft decisions.

Symbol-by-symbol MAP processing utilizes both a priori information (input symbol probabilities) and, as the name implies, a posteriori information (the samples of the received signal). Central to the operation of the algorithm is the fact that the a posteriori information used when computing the symbol probabilities at a given time $t$ includes both the samples received prior to time $t$ as well as those received subsequent to it. 


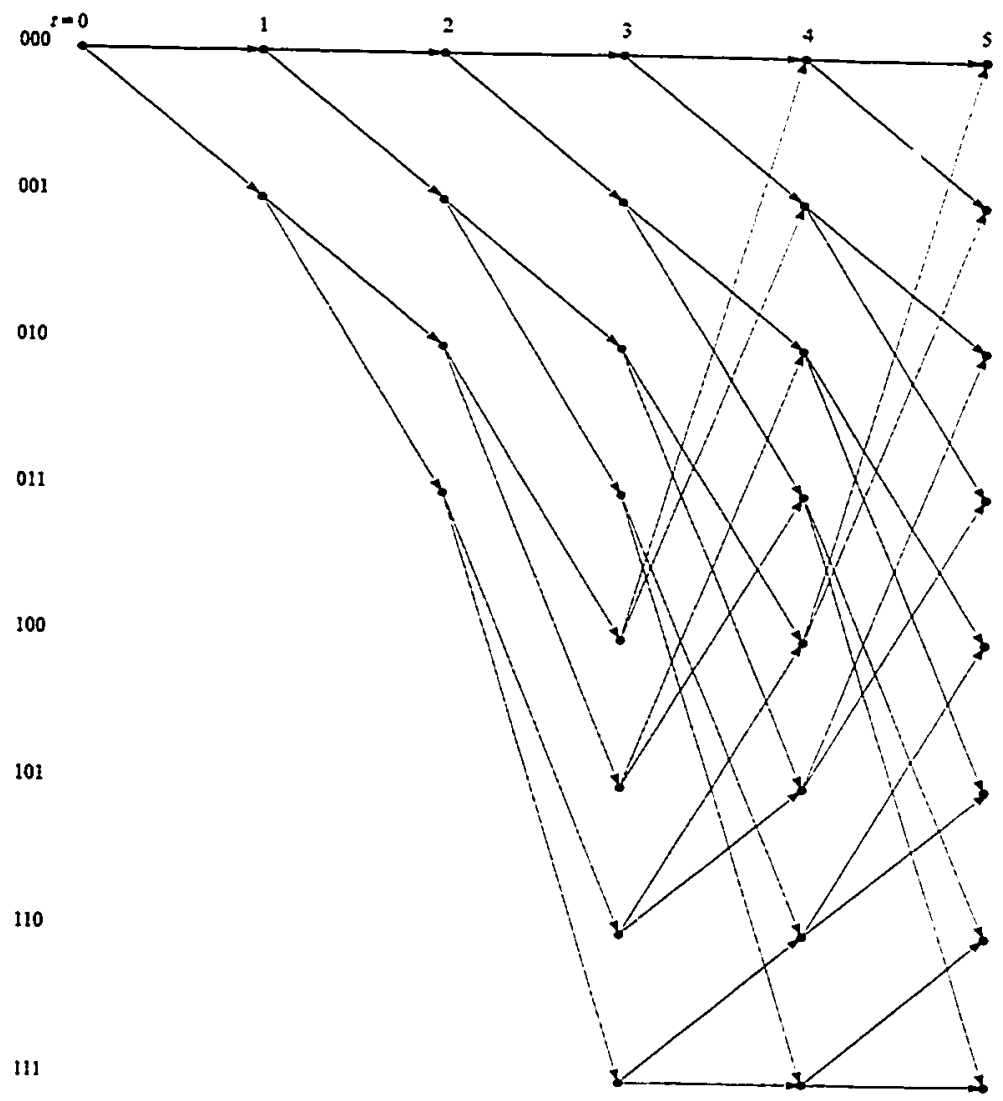

Figure 5-1 A partial trellis for a binary system with 3 bits of memory.

The problem facing the symbol-by-symbol MAP demodulator of a signal with memory (either due to the transmitted pulse shape, the channel or both) can be thought of in terms of a trellis where the state of the trellis represents the bits affecting the received signal at any given time. For example. the trellis shown in Figure 5-1 represents a system with a combined memory of 3 symbol periods, binary modulation, and an initial state of 0 . 
Given the trellis, the goal of the demodulator is then to determine the a posteriori probabilities for each branch. Once all of the branch probabilities are known, it is straightforward to compute the corresponding symbol probabilities.

\subsection{State Transition Probabilities}

Let:

$$
\begin{aligned}
Q & =\text { number of data symbols ( } Q \text { - ary modulation) } \\
N & =\text { length of data block, in symbol periods } \\
M & =\text { number of states in trellis } \\
k & =\text { hypothesis } \\
r & =\text { number of samples/ symbol } \\
t & =\text { discrete time index, in symbol periods } \\
\widetilde{\mathrm{U}} & =N \text { - vector of transmitted symbols } \\
\widetilde{\mathrm{X}} & =r N \text { - vector of transmitred symbol samples } \\
\mathrm{Y} & =r N-\text { vector of received symbol samples }
\end{aligned}
$$

$\mathrm{U}(k)=N$ - vector of $Q$-ary input symbols for hypothesis $k$

$\mathrm{X}(k)=r N$ - vector of transmitted samples for hypothesis $k$

Assume that the $Q$-ary input data symbols are independent. We can then maximize the a posteriori probability of the detected symbols by maximizing

$$
\operatorname{Pr}\{\tilde{\mathbf{U}}=\mathbf{U}(k) \mid \mathbf{Y}\}
$$

such that $k$ is the most likely hypothesis. This probability can be written

$$
\operatorname{Pr}\{\tilde{\mathrm{U}}=\mathrm{U}(k) \mid \mathbf{Y}\}=\frac{p(\mathbf{Y} \mid \overline{\mathrm{U}}=\mathrm{U}(k)) \cdot \operatorname{Pr}\{\tilde{\mathrm{U}}=\mathrm{U}(k)\}}{p(\mathbf{Y})} .
$$

where. from the assumption of independent input symbols.

$$
\operatorname{Pr}\{\tilde{\mathbf{U}}=\mathbf{U}(k)\}=\prod_{s=0}^{N-1} \operatorname{Pr}\left\{\tilde{u}_{t}=u_{t}(k)\right\}
$$

Letting $\mathbf{X}(k)$ represent the vector of transmitted samples corresponding to the input vector $\mathbf{U}(k)$, we can write

$$
p(\mathrm{Y} \mid \overline{\mathrm{U}}=\mathrm{U}(k))=p(\mathrm{Y} \mid \tilde{\mathrm{X}}=\mathbf{X}(k))
$$


From (3) in [33]. we know

$$
\begin{aligned}
& p_{\mathrm{ytx}}[\mathrm{Y} \mid \mathrm{X}(k)]=p_{\mathrm{yt}}\left[\left(y_{N-1}, \cdots, y_{1}, y_{0}\right) \mid \mathbf{X}(k)\right] \\
& =\prod_{j=0}^{N-1} p_{y, y_{j-1}}\left[y_{1}\left[y_{j-1}(k)\right]\right.
\end{aligned}
$$

where

$$
Y_{j}(k)=\left\{\left(y, \cdots, y_{i}, y_{0}\right) \cdot \mathbf{X}(k)\right\}
$$

The computation of $p_{y, w_{-1}}\left[y, \mid y_{j-1}(k)\right]$ is dependent upon the channel model and modulation scheme. For the special case of CPM over a Rayleigh flat-fading channel. the computation will be described in Section 5.5 .

Using (5.2), (5.3) and (5.4), we can rewrite (5.1) as

$$
\begin{aligned}
& \operatorname{Pr}\{\tilde{\mathbf{U}}=\mathbf{U}(k) \mid \mathbf{Y}\}=\frac{\left[\prod_{i=0}^{N-i} \prod_{j=n}^{r(s+1)-1} p_{y_{j}, y_{j-1}}\left[y_{j} \mathbb{Y}_{j-1}(k)\right]\right] \cdot \prod_{i=0}^{N-1} \operatorname{Pr}\left\{\bar{u}_{\mathrm{r}}=u_{i}(k)\right\}}{p(\mathbf{Y})} \\
& =\frac{\prod_{t=0}^{N-1}\left[\operatorname{Pr}\left\{\tilde{u}_{r}=u_{s}(k)\right\} \cdot \prod_{j=n}^{r(t-1)-1} p_{y_{j}, k_{j-1}}\left[y_{j} \mathscr{Y}_{j-1}(k)\right]\right]}{p(\mathbf{Y})}
\end{aligned}
$$

Now consider a trellis which represents a stream of input symbols. For $Q$-ary symbols, each nodz of the trellis has $Q$ inputs and $Q$ outputs. The number of states is given by $M=Q^{K}$ where $K$ is the combined memory, in symbol periods, of the modulator and the channel.

For any given trellis branch, each possible symbol at a given time is uniquely specified by the starting and ending states of the corresponding branch at that time. Therefore, there is a 1-to-1 correspondence between the sequence of input symbols

$$
\mathrm{U}(k)=\left\{u_{0}(k) u_{1}(k) \ldots u_{N-1}(k)\right\}
$$

and the sequence of state transitions

$$
\mathbf{M}(k)=\left\{\left(m^{\prime}, m\right)_{0}\left(m^{\prime}, m\right)_{1} \ldots\left(m^{\prime}, m\right)_{N-1}\right\} .
$$

where the $\left\{\left(m^{\prime}, m\right)_{s}\right\}$ represent the starting and ending states for the $k$ th hypothesis at 
time $t$, and $m^{\prime}$ at time $t$ is the same as $m$ at time $t-1$. Using this correspondence, we can define

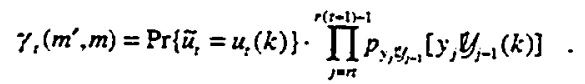

The $\left\{\gamma_{1}\left(m^{\prime}, m\right)\right\}$ can be seen to be weights associated with each branch of the trellis. While the sequence of multiplicative branch weights is a function of the hypothesis $k$. individual multiplicative branch weights, $\gamma_{s}\left(m^{\prime}, m\right)$, are not. For the first term of (5.6), because each branch is associated with a specific symbol. all hypotheses that pass through a given branch will include the specific symbol in that time period. For the second term, since the computation of $p_{y, y_{j-1}}\left[y_{j}, Y_{j-1}(k)\right]$ is dependent upon at most $K+1$ symbols, corresponding to a trellis with $Q^{k}$ states, it is also independent of $k$.

We are interested in the probability that a given branch was traversed at time $t$ since this can be used to determine the probability that a given symbol was transmitted at time $t$. Define the set $C_{t}(m: m)$ as the set of hypotheses $\{k\}$ that traverse the trellis branch between states $S_{i-1}=m^{\prime}$ and $S_{i}=m$. The state transition probability is then given by

$$
\operatorname{Pr}\left\{S_{t-1}=m^{\prime} ; S_{r}=m \mid \mathbf{Y}\right\}=\frac{\sum_{k \in C_{i}\left(m^{\prime} ; m\right)} \operatorname{Pr}\{\tilde{\mathrm{U}}=\mathrm{U}(k) \mid \mathbf{X}\}}{\sum_{k} \operatorname{Pr}\{\overline{\mathrm{U}}=\mathrm{U}(k) \mid \mathrm{Y}\}} .
$$

Substituting (5.5) and (5.6) into (5.7) allows the state transition probability to be written

$$
\operatorname{Pr}\left\{S_{r-1}=m^{\prime}: S_{i}=m \mid \mathbf{Y}\right\}=\frac{\sum_{k a C_{1}\left(m^{\prime}, m\right)} \prod_{i=0}^{N-1} \gamma_{i}\left(\mathbf{M}_{i}(k)\right)}{\sum_{k} \prod_{i=0}^{N-1} \gamma_{i}\left(\mathbf{M}_{i}(k)\right)}
$$

where $\mathrm{M}_{i}(k)$ denotes the ith element of the hypothesized path through the trellis, $\mathbf{M}(k)$.

\subsection{Efficient Computation Of The State Transition Probabilities}

The quantity on the right-hand side of $(5.8)$ is recognized to be a generic computational problem. That is. for each branch in the trellis. we wish to compute the ratio 


\section{Sum of the products of the weights of all paths through the trellis which pass through the given branch \\ Sum of the products of the weights of all paths through the trellis}

This section briefly describes a computationally efficient approach to the above problem.

As an example. consider the length $N=7$ trellis shown in Figure 5-2(a) where the nodes correspond to one of $M=2$ states and the branches represent possible transitions.

Assume that we wish to compute the sum of the products of the weights of all paths through the trellis (there are $2^{6}$ paths which start and end in state 0 ). An efficient way to perform this computation is via a recursion of the form

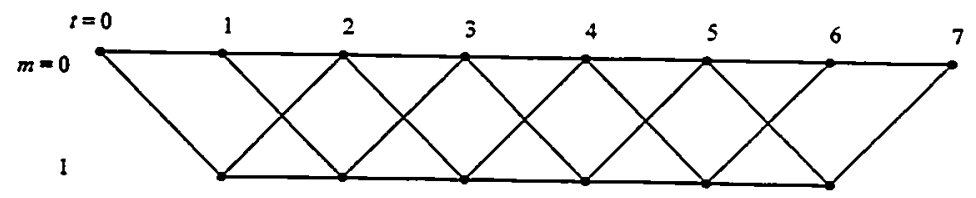

(a)

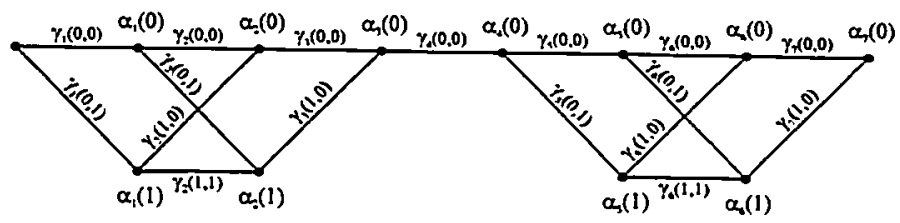

(b)

Figure 5-2 (a) The trellis of the example Markov process. (b) The equivalent trellis when only those paths passing through the branch from state $S_{3}=0$ to state $S_{4}=0$ are considered. 


$$
\alpha_{t}(m)=\sum_{m^{\prime}} \alpha_{t \rightarrow 1}\left(m^{\prime}\right) \cdot \gamma_{t}\left(m^{\prime}, m\right)
$$

where $\alpha_{f}(m)$ is the sum of the products of the weights along all paths which terminate in state $m$ at time $t$ and $\alpha_{0}(m), 0 \leq m \leq M$, are known initial conditions. The sum over $m^{\prime}$ is for all cases for which a branch exists from state $m^{\prime}$ to state $m$ at time $t$, and $\gamma_{t}\left(m^{\prime}, m\right)$ is the corresponding branch weight.

Now consider the case where we wish to solve for the sum of the procucts of the iveights for only those paths which traverse a particular branch in the trellis. In this case, it is advantageous to split the trellis into two parts. This is shown in Figure 5-2(b) for the case of the branch from $S_{3}=0$ to $S_{2}=0$. From $(5.9), \alpha_{3}(0)$ gives us the sum of the products of the weights along all subpaths which terminate in state 0 at time $t=3$. The weight on the branch itself is $\gamma_{4}(0.0)$. To compute the summation for the second half of the trellis, a backward recursion can be used. This recursion has the form

$$
\beta_{t}(m)=\sum_{m^{\prime}} \beta_{t+i}\left(m^{\prime}\right) \cdot \gamma_{t+1}\left(m . m^{\prime}\right)
$$

where $\beta_{z}(m)$ is the sum of the products of the weights along all paths to the end of the trellis which originate from state $m$ at time $t$ and $\beta_{N}(m) .0 \leq m \leq M$, are known final conditions. Here, the sum over $m^{\prime}$ is for all cases for which a branch exists from state $m$ to state $m^{\prime}$ at time $t$, and $\gamma_{t}\left(m . m^{\circ}\right)$ is the corresponding branch weight.

In general, if we define $\sigma_{z}\left(m^{\prime}, m\right)$ as the sum of the products of the weights of all paths which pass through the branch delineated by $S_{j \cdot l}=m^{\prime}$ and $S_{z}=m$, then

$$
\sigma_{r}\left(m^{\prime}, m\right)=\alpha_{t-1}\left(m^{\prime}\right) \cdot \gamma_{t}\left(m^{\prime} \cdot m\right) \cdot \beta_{z}(m) \text {. }
$$

Note that to solve (5.11) for all branches of the trellis. the forward and backward recursions (over the whole trellis) only need to be performed once.

Equations (5.9), (5.10) and (5.11) represent an efficient recursive approach for addressing the generic computational problem posed at the beginning of this subsection. The form 
of this approach is essentially that of the algorithm by Bahl et al [12] which was described in Section 2.4 but differs from it in two important respects:

a) While the Bahl algorithm was derived for the specific case of probabilities governing a discrete-time finite-state Markov process. in this thesis it is recognized that the algorithm in [12]. and others like it. are simply efficient ways to solve the generic computation problem stated at the beginning of this section.

b) The definitions of $\alpha_{z}(m)$ and $\beta_{t}(m)$ presented herein are symmetric while those in [12] are asymmetric.

If the initial and final states are not known, the algorithm can operate in a block-by-block mode where the value of $\alpha_{6}(m)$ is obtained from the $\alpha_{s}(m)$ of the previous block. and the value of $\beta_{s}(m)$ is obtained by assuming equiprobable final states and overlapping the processing into the next block. The blocks must be sufficiently overlapped to ensure that the transients due to estimation errors do not significantly affect performance.

\subsection{Computation Of Soft Decisions}

For demodulation purposes. the quantity of interest is $\operatorname{Pr}\left\{\bar{u}_{t}=q \mid \mathbf{Y}\right\}$ where $q$ is one of the $Q$-ary input symbols. This quantity is calculated by summing the transition probabilities that correspond to branches associated with the symbol $q$ at time $t$.

Define a set of all state transitions for which $\tilde{u}_{r}=q$.

$$
A=\left\{\left(m^{\prime} \cdot m\right): \bar{u}_{t}\left(m^{\prime}, m\right)=q\right\} .
$$

where $\tilde{u}_{t}\left(m^{\prime}, m\right)$ is the transmitted symbol corresponding to the branch from state $S_{f-1}=m^{\prime}$ to state $S_{s}=m$. Then

$$
\operatorname{Pr}\left\{\bar{u}_{r}=q \mid \mathrm{Y}\right\}=\sum_{\left(m^{\prime} ; m\right) \in A} \operatorname{Pr}\left\{S_{t-1}=m^{\prime}: S_{t}=m \mid \mathbf{Y}\right\} .
$$

Making use of the fact that the numerator of (5.8) is given by $\sigma_{i}\left(m^{\prime}, m\right)$, the desired probability is then 


$$
\operatorname{Pr}\left\{\tilde{u}_{z}=q \mid \mathrm{Y}\right\}=\frac{\sum_{\left(m^{\prime}, m\right) A^{\prime}} \sigma_{z}\left(m^{\prime}, m\right)}{\sum_{\left(m^{\prime}, m\right)} \sigma_{f}\left(m^{\prime}, m\right)} .
$$

Note that the denominator of (5.13) is in fact the sum of the products of the weights of all paths through the trellis and is therefore the same at all times $t$. In fact. from the definition of $\alpha_{t}(m),(5.13)$ can also be written

$$
\operatorname{Pr}\left\{\bar{u}_{t}=q \mid \mathbf{Y}\right\}=\frac{\sum_{\left(m^{\prime}, m / \mu_{1}\right.} \sigma_{r}\left(m^{\prime}, m\right)}{\sum_{m} \alpha_{N}(m)}
$$

where the summation in the denominator is over the possible final states of the trellis.

\subsection{Computation Of Probability Density}

From (5.9) through (5.13), we can see that the computation of the branch probabilities (and thus the symbol probabilities) revolves around the determination of $\gamma_{t}\left(m^{\prime}, m\right)$ and the structure of the trellis. The trellis is straightforward since it is simply determined by the bit (or symbol) sequence which is affected by the memory of the modulation and the channel. The value of $\gamma_{t}\left(m^{\prime}, m\right)$ is a function of the $a$ priori symbol probabilities and the probability distribution function of the received channel samples conditioned on the input and the previously received samples. i.e., $p_{y_{j} z_{j-1}}\left[y_{j} \mid y_{j-1}\left(m^{\prime}, m\right)\right]$.

While the results of sections 5.2 through 5.4 are generally applicable to the demodulation of signals in any sysem which has memory, we now restrict ourselves to the case of CPM signals transmitted over Rayleigh flar-fading channels. Consider a system such as that shown in Figure 5-3(a) where $x_{r}(k)$ represents the transmitted symbol sample for hypothesis $k$ at time $n$. S(f) represents the power spectral density of the fading process filter and $y_{m}(k)$ represents the corresponding received symbol sample. If the receiver removes the modulation corresponding to hypothesis $k$, we are left with $y^{\prime},(k)$ which represents the combined effect of the multiplicative fading and the additive noise. Note that removing the modulation is a linear transformation with a Jacobian of magnitude one for CPM. Therefore. 


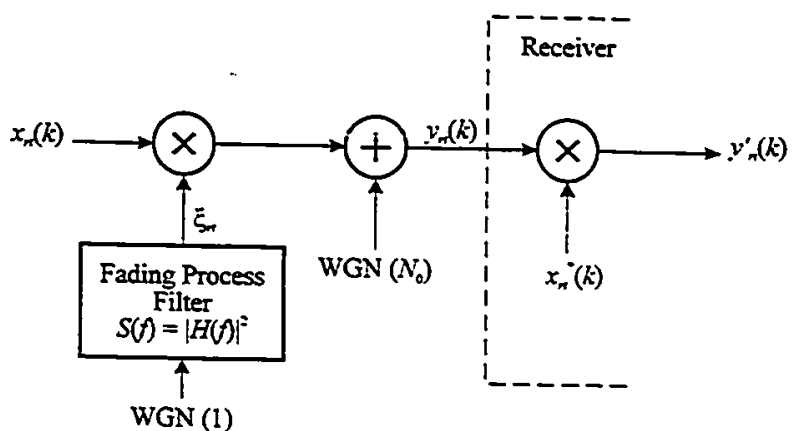

(a)

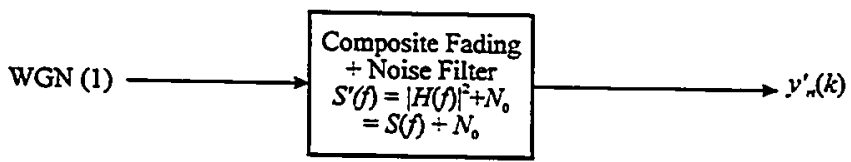

(b)

Figure 5-3 Statistically equivalent alternate transmission models (from [33]).

$$
p_{Y^{\prime} \mathrm{XX}}\left[\mathbf{Y}^{\prime}(k) \mid \mathbf{X}(k)\right]=p_{\mathrm{YIX}}[\mathbf{Y} \mid \mathbf{X}(k)]
$$

In [33]. it is shown that for constant envelope signals whose normalized signal amplitude is one (for convenience only). $\mathrm{Y}^{\prime}(k)$ is a zero mean complex Gaussian process with covariance $R$ where $R$ is the Toeplitz covariance matrix whose elements correspond to the autocorrelation coefficients of the composite fading plus noise power spectral density function $S^{\prime}(f)$ shown in Figure 5-3(b). The conditional pdf of $Y^{\prime}(k)$, the fading plus noise. given the input $\mathbf{X}(k)$ can then be written as

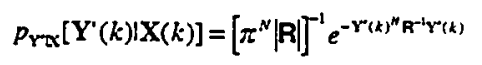


where $(-)^{H}$ represents a Hermetian transpose.

The representation shown in Figure 5-3(b) suggests a receiver structure which uses a predictor to estimate the composite fading plus noise process. Theorem I of [33] shows how to compute $\mathbf{R}$ from the outputs of an all-pole linear predictor. given a hypothesized transmitted sequence. Theorem 2 of [33] shows that

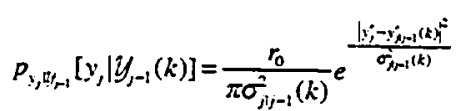

where

$$
\begin{aligned}
& y_{j,-1}^{\prime}(k)=\sum_{i=1}^{i-1} a_{i}^{j-1} y_{j-i}^{\prime}(k), \\
& \sigma_{j,-1}^{\prime}(k)=r_{0} V_{j-1} .
\end{aligned}
$$

$a_{i}^{\prime-1}$ is the ith coefficient of the $(j-1)$ th-order linear predictor, $V_{j-1}$ is the corresponding expected normalized squared prediction error $\left(V_{0}=1\right)$ and $r_{0}$ is the zero-lag autocorrelation coefficient.

If it is assumed that the composite fading plus noise filter can be accurately approximated by an all-pole model of order $L$. all linear predictors of order $L$ and greater have essentially the same coefficients and therefore approximately the same expected normalized squared prediction error. Assuming a known preamble. the contribution of predictor orders less than $L$ can be ignored. Therefore, using (5.17), (5.18). (5.19) and the fact that $V_{j-1} \approx V_{L}$ for $j>L$. we can rewite (5.6) as 


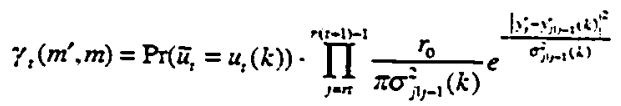

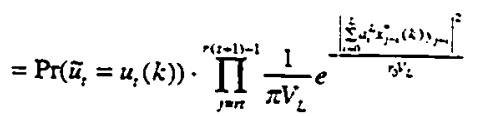

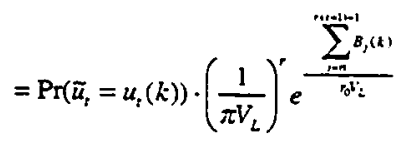

where

$$
B_{,}(k)=\left|\sum_{i=0}^{L} a_{1}^{2} x_{j-i}^{-}(k) y_{j-i}\right|^{2}
$$

and $a_{0}^{L}=-1$. Note that the summation term in (5.21) represents the output of a prediction error filter for $y^{\prime}$ combined with the removal of the hypothesized modulation over a sequence of $L$ received samples.

\subsection{Implementation}

In this section. we derive a receiver structure suitable for feeding the symbol-by-symbol MAP processing.

Since the computation of the soft decision (5.13) utilizes a ratio of $\sigma_{t}\left(m^{\prime}, m\right)$ terms, and since each summation term comprising $\sigma_{t}\left(m^{\prime}, m\right)$ is a product of $N$ factors of the form of (5.20), the common factor $\left(\pi V_{L}\right)^{-r}$ in (5.20) can be omitted. yielding

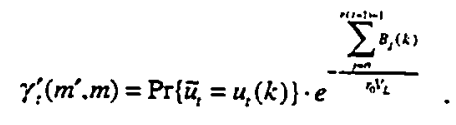

The MAP recursions described in Section 5.3 can then be used to produce soft decisions by substituting $\gamma_{s}^{\prime}\left(m^{\prime}, m\right)$ for $\gamma_{s}\left(m^{\prime}, m\right)$ in (5.9) through (5.11). 
The calculation of the exponential term of (5.22) can be implemented using the matched filter bank shown in Figure 5-4. Each filter combines the removal of the hypothesized modulation with the prediction stror filter function required for the computation of $B_{j}(k)$. The form and length of these filters is derived in [33] and in Appendix A. It is shown that all calculations required for the computation of the $B_{j}(k)$ 's for a single symbol period are affected by no more than $\lceil\kappa+\chi\rceil$ symbols where $\kappa$ represents the pulse lenç, th in

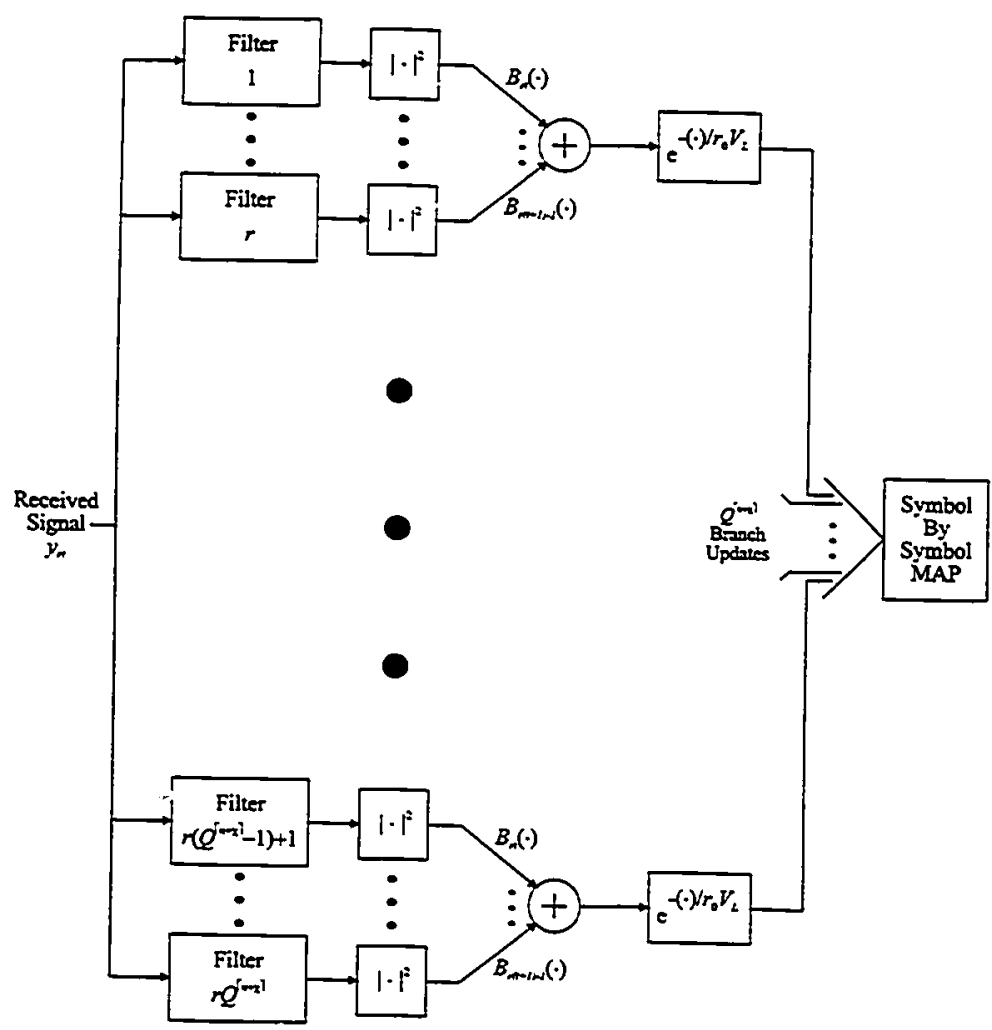

Figure 5-4 The structure of the filter bank used by the MAP demodulator. 
symbol periods and $\chi$ represents the predictor length in symbol periods. Since this span of symbols represents the memory required in the demodulator, the number of states required for $Q$-ary modulation is given by $Q^{[\kappa-z ;-1}$. Note that the " -1 " term results from the fact that the current symbol does not need to be stored in the memory of the demodulator.

Referring again to Figure 5-4. the output of the filters for a given symbol period and hypothesis are combined (if the number of samples/symbol is greater than 1 ). the result divided by the expected prediction error $r_{0} V_{L}$. and then the exponent taken. This salue is then multiplied by the probability of the current hypothesized symbol. resulting in $\gamma_{,}^{\prime}\left(m^{\prime} \cdot m\right)$ as specified in (5.22).

A few points should be noted:

- the amount of storage required is proportional to the number of states, and to the length of the trellis in symbols $(N)$ :

- the computational complexity is dominated by the number of states:

- because the computation of the $B_{j}(k)$ terms involves the magnitude of the matched filter output. the number of filters for a given modulation scheme may be reduced by identifying filters which will produce the same output.

For a given composite fading plus noise power spectral density, the matched filters are FIR filters with fixed coefficients. Under changing channel conditions, these filters will have to be updaied periodically by some sort of adaptive channel estimator. 


\section{Chapter 6}

\section{Demodulation Performance}

\subsection{Description Of Simulation}

In order to evaluate the performance of the symbol-by-symbol MAP algorithm. support for symbol-by-symbol MAP processing was added to CRCs Linksim simulation

program. This program is written in ANSI C and runs on PC compatible computers.

The structure of the communications link simulated by Linksim is shown in Figure 6-1. A random bit stream is generated and passed to the transmiter. The sampled signal produced by the transmitter is passed through a communications channel where various impairments are applied before the signal is passed to the receiver. An error counter computes an enror rate by comparing the bit stream generated by the receiver with that fed to the transmitter.

In order to ensure that simulation results are statistically valid, several suns are performed. each producing at least 100 error events. No reinitialization is performed between runs: this ensures that the random number generators which drive the simulation

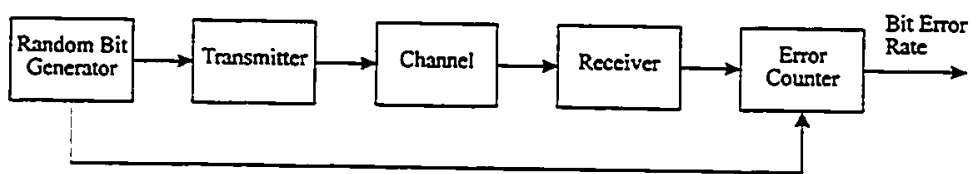

Figure 6-1 A block diagram of the simulation. 
produce independent values for each run. As each run is completed. the mean BER for that run, and the mean BER for all runs-to-date are output. When all runs have been completed, the final mean BER and a 9S\% confidence interval are output. The statistical basis for the confidence interval is described in Appendix B - Simulation BER Confidence Intervals.

\subsubsection{Transmitter}

A block diagram of the transmitter is shown in Figure 6-2. The input bit stream is optionally convolutionally encoded and block interleaved (bit-by-bit). Framing. a sequence of known bits repeated once per frame. may also be added at this point. The resulting bit stream is then mapped into a selected signal constellation (a complex phasor). If symbol interleaving is required, the symbols may be block interleaved (symbol-by-symbol). They are then upsampled to the desired sample rate by inserting the appropriate number of zero samples between successive input samples. The resulting samples may be passed through a pulse shaping filter and/or a CPM modulator. No

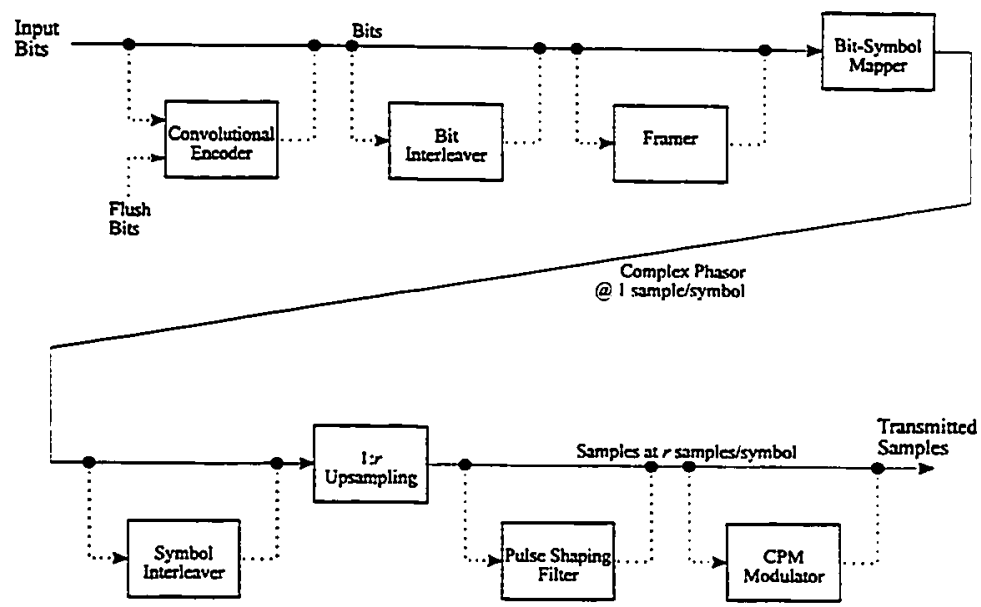

Figure 6-2 A block diagram of the simulated transmitter. 
additional transmit filtering is performed.

\subsubsection{Pulse Shaping}

The pulse shape utilized for the binary CPM simulations is the N32FM pulse shape described in [19]. This pulse shape satisfies Nyquist's second and third criteria for no intersymbol interference and, because CPM is inherently differentially encoded, can be effectively demodulated via differential detection. Figure 6-3 illustrates the pulse shape truncated to three symbol periods, and the resulting power spectral density. As can be seen, the resulting modulated signal is bandwidth efficient, with an asymptotic rolloff proportional to $l /\left|f-f_{c}\right|^{b}$.

\subsubsection{Block Processing}

The symbol-by-symbol MAP algorithm is inherenty a block-oriented algorithm. It operates on a block of samples. with known initial and final states. The algorithm can be adapted to a continuous stream of data by utilizing the decoded state from a previously processed block: however, in the simulations performed for this thesis, $\log _{2}(M)$ known framing bits (all zeroes) are inserted every 1024 data bits to provide the known initial and final states. This is representative of a system. such as the Inmarsat Aeronautical system [34], which has frames delimited by known unique words.

A block length of 1024 bits minimizes the positive effect that the known initial and final states will have on the performance of the algorithm (when compared with the performance of the non-block-oriented approaches) while keeping the memory requirements manageable.

Figure 6-4 illustrates the framing structure implemented for the symbol-by-symbol MAP simulations. 


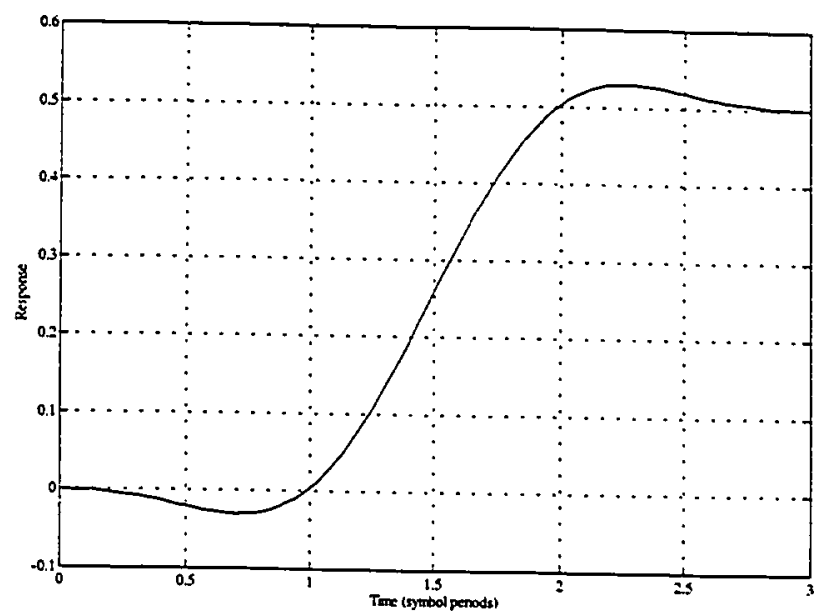

(a)

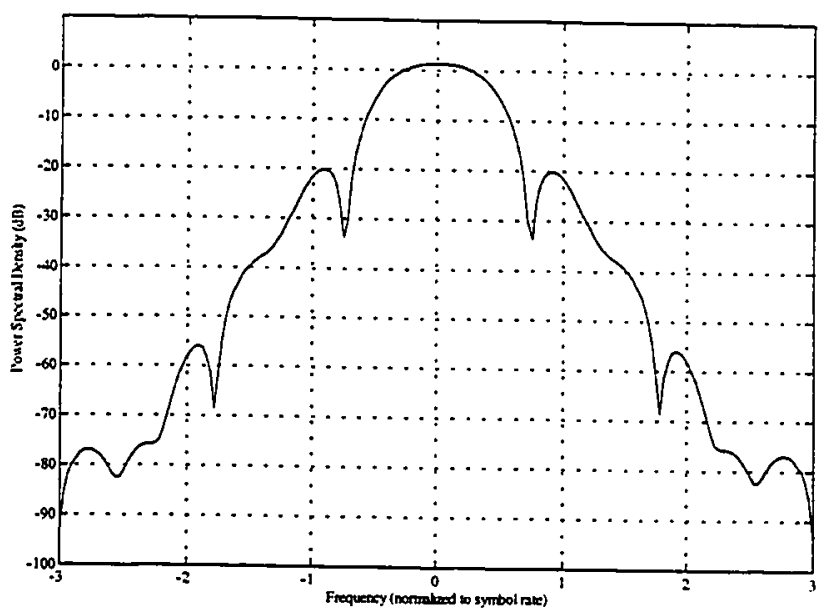

(b)

Figure 6-3 The (a) phase pulse and (b) power spectrum of N32FM. 


\begin{tabular}{|c|c|c|c|c|}
\hline $\begin{array}{c}\text { Framing } \\
\text { Bits }\end{array}$ & Data & $\begin{array}{c}\text { Framing } \\
\text { Bits }\end{array}$ & Data & $\cdots$ \\
\hline
\end{tabular}

Figure 6-4 The frame structure used for the MAP simulations.

\subsubsection{Interleaving}

As illustrated in Figure 6-2, either bit interleaving or symbol interleaving may be utilized. While the two are equivalent for binary modulations, they differ for higher order modulations. Consider the case of rate-1/2 coded QPSK. For every bit which goes into the convolutional encoder, two bits come out. If bit interleaving is performed, these two bits are encoded in separate symbols, and are thus separated in time during transmission. If symbol interleaving is performed, the two bits form a single symbol, and are therefore interleaved together. From the perspective of the convolutional decoder, bit interleaving spreads the effect of an unreliable symbol while symbol interleaving concentrates it at a single node in the trellis.

\subsubsection{Channel}

A block diagram of the channel is shown in Figure 6-5. The fading process is generated by passing white gaussian noise (WGN) through a lowpass filter (hereinafter referred to as the fading filter). Depending upon the design of the fading fiiter, the resulting samples may be interpolated. This allows low fading bandwidths to be generated with reasonable lengths of fading filters: in effect. the interpolation function scales the cutoff frequency of the fading filter down by a factor equal to the amount of interpolation. The resulting fading process samples are multiplied by the input samples and then white gaussian noise is added to produce the appropriate $E_{b} / N_{0}$. Input samples are assumed to have unity power. 


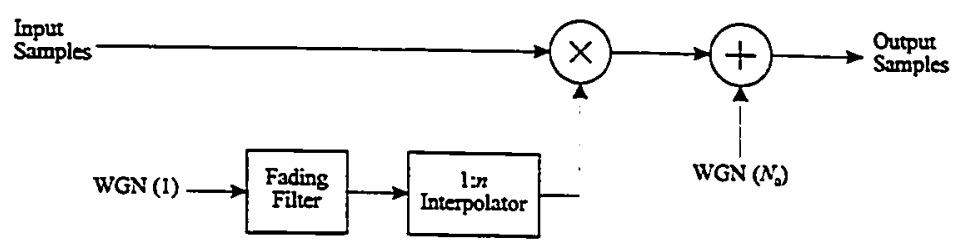

Figure 6-5 A block diagram of the simulated channel.

The fading filters utilized in this work are finite impulse response (FIR) filters which are generated as follows:

- a sampled spectral raised cosine with the required curoff frequency is generated in the frequency domain (Figure 6-6a):

- an inverse FFT is performed to obtain time domain samples; due to the symmetry of the frequency response. the result is real:

- the time domain samples are rearranged in the appropriate order (Figure 6-6b);

- the impulse response is truncated to the desired length: the coefficients for a length $l$ filier are the center $l$ samples (Figure 6-6c).

The frequency response of a 79 tap filter designed for a cutoff frequency of $0.3 R$ at $r=2$ samples/symbol and no interpolation is shown in Figure 6-6d.

There are an infinite variety of fading spectra which could be chosen as representative of the channel of interest. In this thesis, the spectral raised cosine shape has been chosen.

This shape provides a relatively uniform distribution of the fading process energy across the fading bandwidth. While alternative spectral shapes might lead to different performance based on the distribution of the energy in the fading process, the comparative results should be valid for any spectrum which can be reasonably modelled by a linear predictor. 


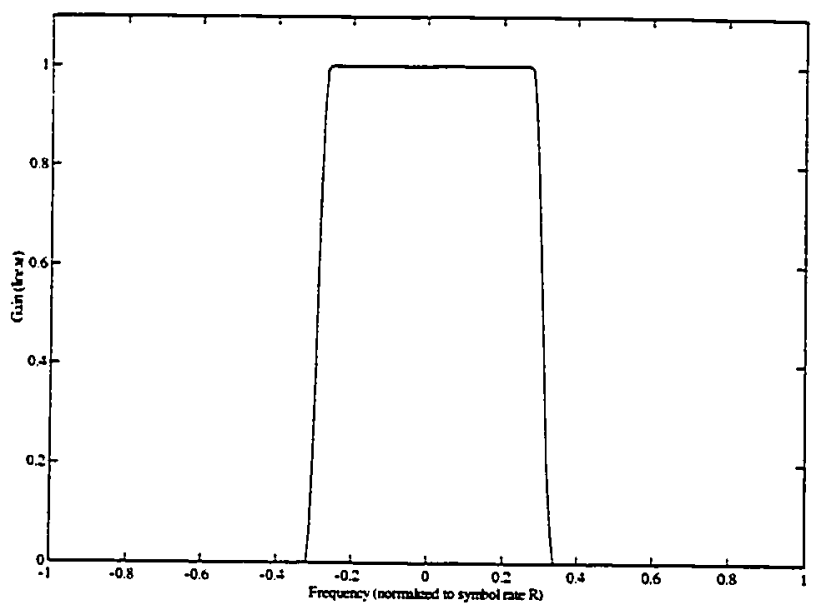

(a) The frequency response of a 256 point spectral raised cosine fading filter.

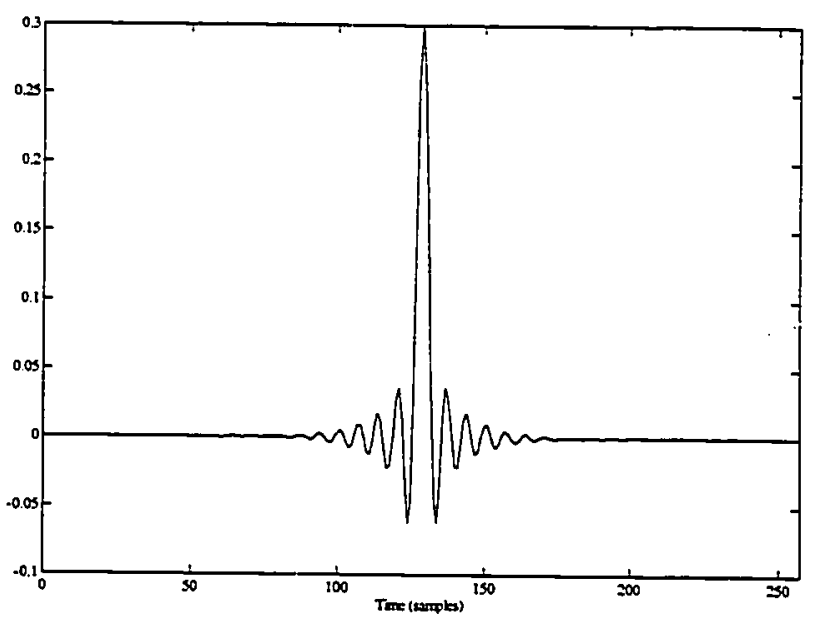

(b) The impulse response of the 256 point spectral raised cosine. 


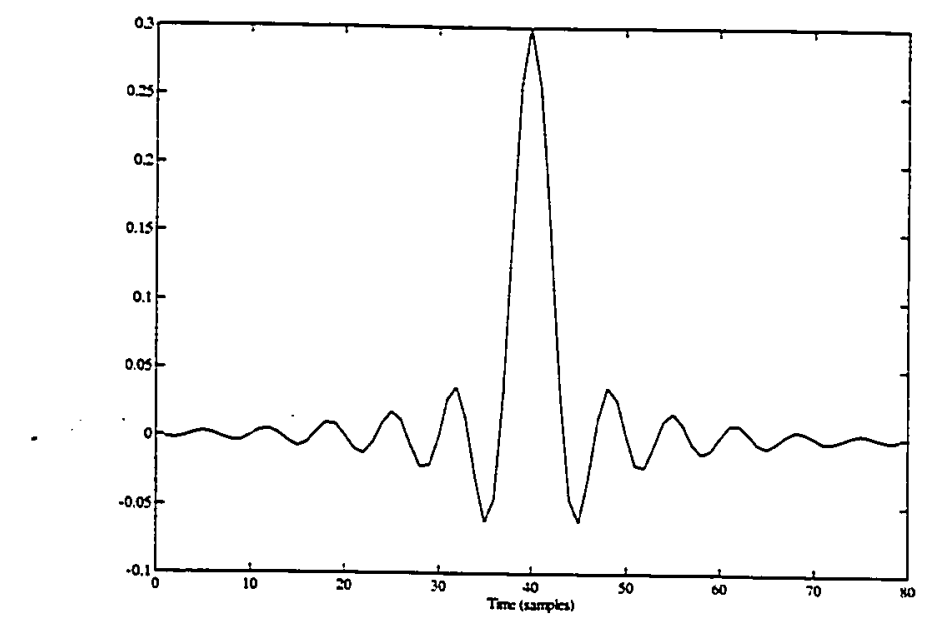

(c) The impulse response after truncation to 79 samples.

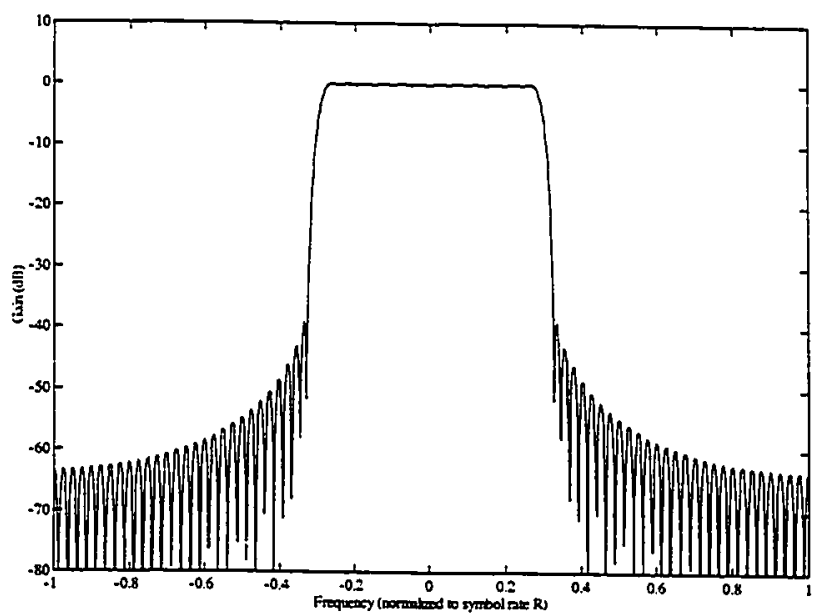

(d) The frequency response of the length 79 fading filter.

Figure 6-6 The impulse and frequency responses of the fading filter. 


\subsubsection{Receiver}

Three different receivers are implemented. This allows the performance of the MAP receiver to be compared to that of the linear predictive (MLSE) receiver [33] and that of conventional differential derection. The overail structure of the simulated receiver is shown in Figure 6-7. The operation of the three receiver types is described below.

\subsubsection{Differential Detector}

The received signal at $r$ samples/symbol is sampled at the mid-symbol time and then passed through a differential detector. The resulting phasor may be passed through a symbol deinterleaver and convolutional decoder, or a hard decision may be made.

From [23], the decision rule for conventional differential PSK is

$$
\text { choose } \Delta \hat{\varphi}_{j} \text { if } \operatorname{Re}\left\{y_{j} y_{j-1}^{\cdot} e^{-j \Delta \dot{\phi}_{j}}\right\} \text { is maximum }
$$

where $y_{j}$ is the received sample for the $j$ th symbol. and $\Delta \hat{\varphi}_{j}$ is the hypothesized

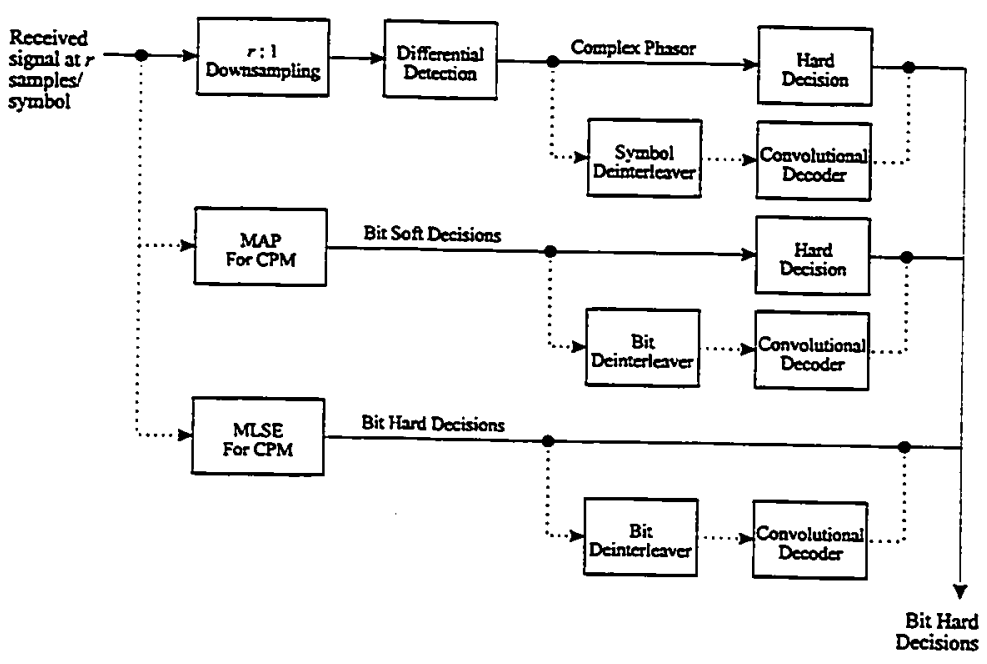

Figure 6-7 A block diagram of the simulated receiver. 
differentially encoded phase shift. If convolutional decoding is required, the corresponding metric for the Viterbi algorithm is

$$
\begin{aligned}
\eta_{j} & =\left|y_{j-1}+y_{j} e^{-j \Delta \dot{\theta}_{j}}\right|^{2} \\
& =\left|y_{j-1}\right|^{2}+\left|y_{j}\right|^{2} \div 2 \operatorname{Re}\left\{y_{, j} y_{j-1}^{\dot{j}_{1}} e^{-\Delta_{1}}\right\}
\end{aligned}
$$

Ignoring those terms in (6.1) which are common to each metric, we get

$$
\eta_{3}=\operatorname{Re}\left\{y_{j} \dot{y}_{j-1}^{*} x_{j}^{-}\right\}
$$

where $x_{j}=e^{-j \Delta \bar{p}_{1}}$ is the phasor corresponding to the branch of interest. Note that no knowledge of the channel state is utilized.

6.13.2 Linear Predictive Receiver (MLSE)

The MLSE algorithm operates on the received signal at $r$ samples/symbol and is implemented for binary CPM only. The algorithm produces bit hard decisions only. The hard decision output may optionally be passed through a bit deinterleaver and convolutional decoder.

\subsubsection{Symbol-By-Symbol MAP Demodulator}

A detailed block diagram of the simulated symbol-by-symbol MAP demodulator is shown in Figure 6-8. Processing stars by collecting a complete frame of samples (framing symbols + data symbols + framing symbols for the next frame) at $r$ samples/symbol. For purposes of the simulation. the modulation is stripped off of the biuck of samples and the value of the expected squared prediction error $E\left[\left|r_{0} V_{L}\right|\right]$ is computed. Altematively, the expected squared prediction error is computed by multiplying the estimated autocorrelation of the received block, $\hat{r}_{0}$ by the normalized prediction error corresponding to the prediction error fitter (PEF) coefficients, $V_{L}$. 


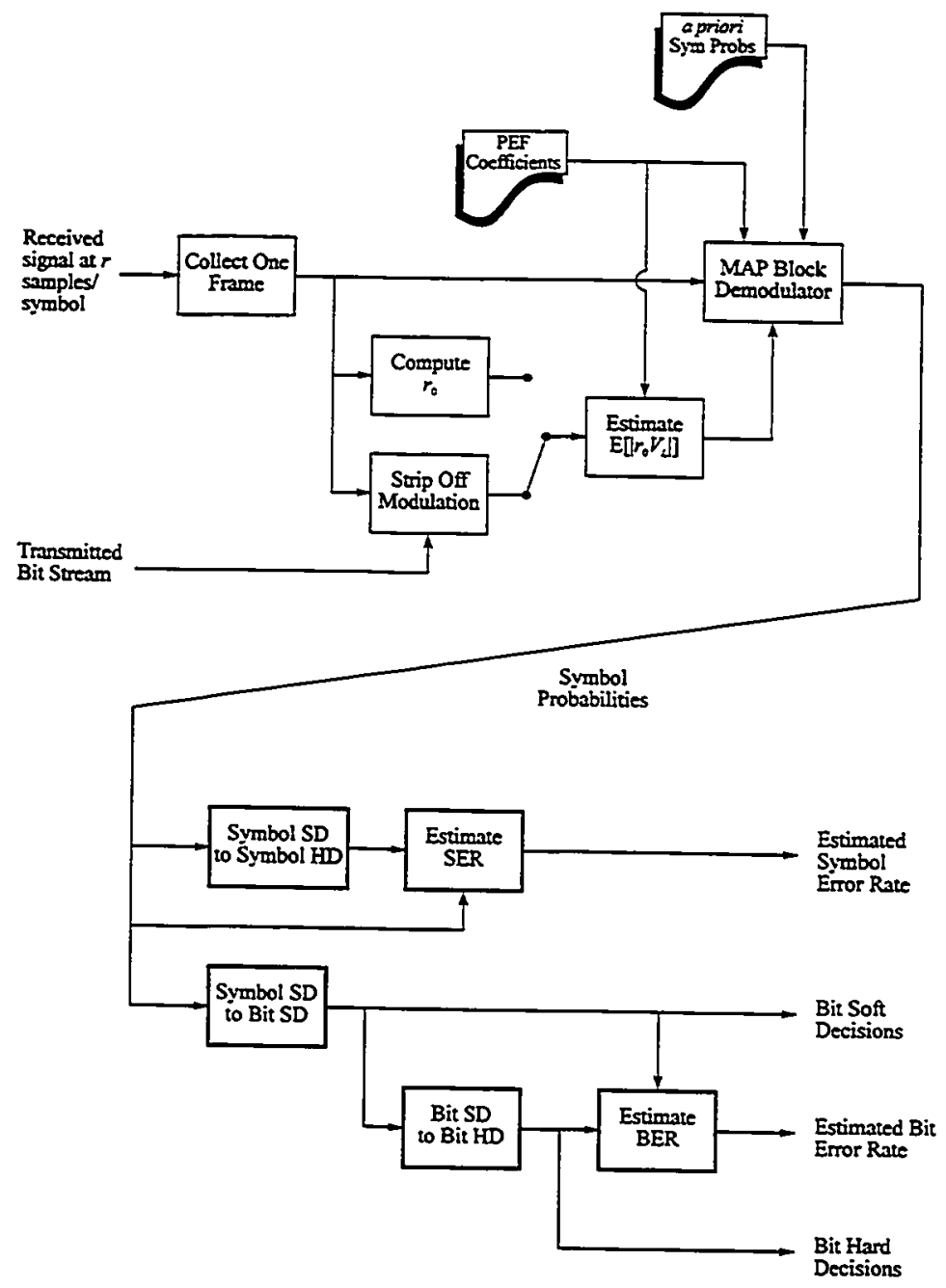

Figure 6-8 A block diagram of the simulated MAP demodulator. 
The compured squared prediction error, received samples. PEF coefficients and the a priori symbol probabilities are then passed to the MAP block demodulator. This element performs the symbol-by-symbol MAP demodulation described in Chapter $5: a$ flowchar of its processing is given in Figure 6-9. Its outputs are a set of symbol probabilities for the data symbols in the block.

The symbol probabilities are then processed to produce:

- bit soft decisions:

- bit harc decisions:

- an estimate of the symbol error rate: and.

- an estimate of the bit error rate.

The bit soft decision, $\mu_{t}$, is defined as the probability that the bit transmitted at time $t$ was a 1, i.e.,

$$
\mu_{i}=\operatorname{Pr}\left(u_{i}=1\right)
$$

For binary CPM. the symbol probabilities map 1:1 to bit soft decisions. For higher order modulations. the bit soft decisions are computed by summing the probabilities at time $t$ for each symbol which has a I in the bit position of interest.

Once a block of $N$ bit soft decisions are available. the block bit error rate is estimated from the soft decisions using

$$
\psi=0.5-\frac{1}{N} \sum_{i=0}^{N-1}\left|\mu_{i}-0.5\right| .
$$




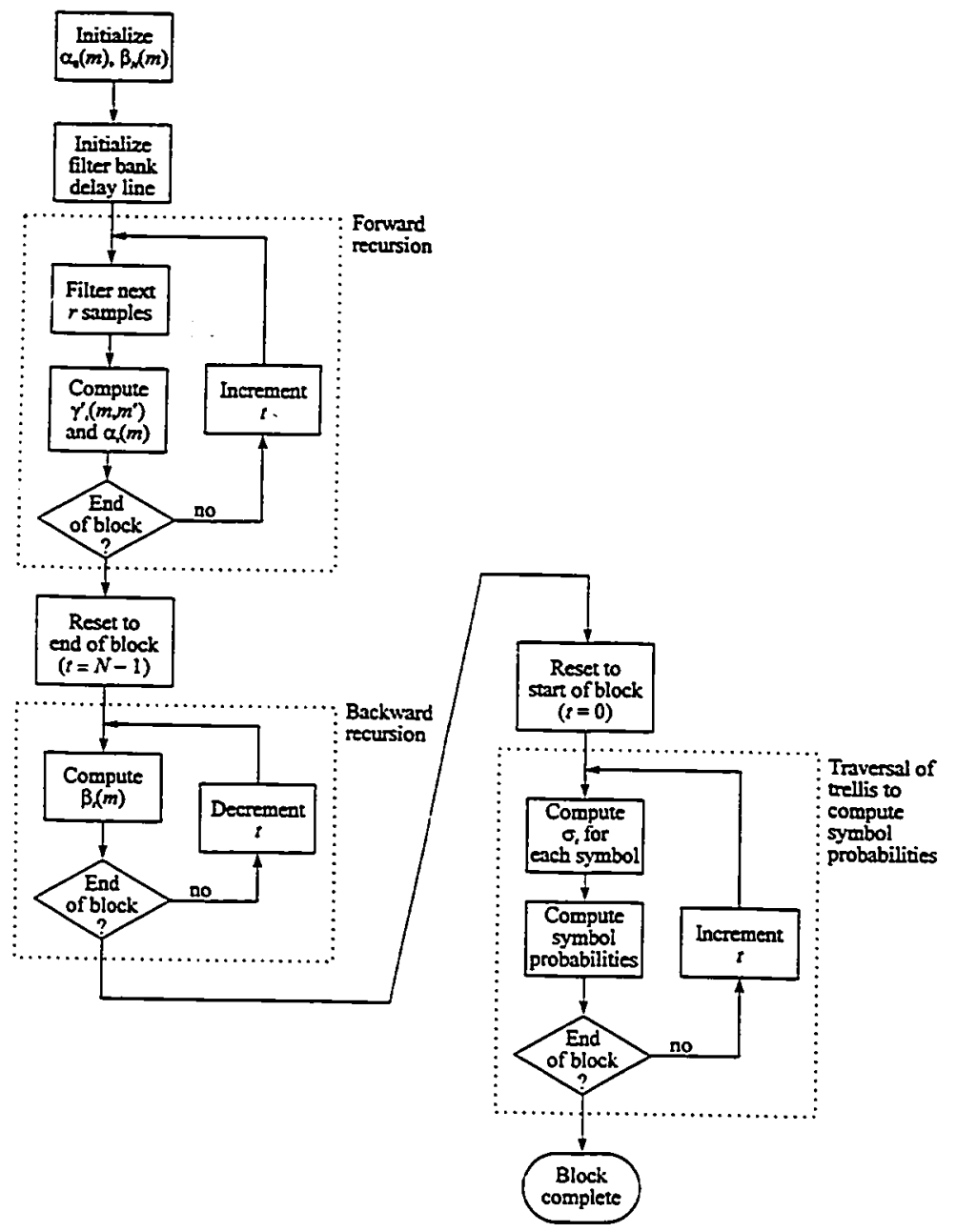

Figure 6-9 A flowchart of the symbol-by-symbol MAP processing. 


\subsubsection{Prediction Error Filters}

Both the linear predictive (MISE) receiver and the symbol-by-symbol MAP demodulator assume that the fading channel may be modelled by an all-pole filter. As shown in (5.21). the quantity of interest is in fact the prediction error. This section illustrates the computation of the prediction error filter coefficients for the case where the predictor is matched to the channel (within the bounds of the predictor order).

Consider the case of a FIR fading filter (generated as per Section 6.1.2) that is to be modelled with an order $L$ predictor. Since the filter is deterministic, its autocorrelation coefficients can be easily computed. Defining the ith lag normalized autocorrelation for the filter as

$$
\rho_{i}=\frac{r_{1}}{r_{0}}
$$

we can write the normal equations of (2.10) as

$$
\left[\begin{array}{ccccc}
\rho_{0}+N_{0} & \rho_{1} & \rho_{2} & \cdots & \rho_{L-1} \\
\rho_{1} & \rho_{0}+N_{0} & \rho_{1} & \cdots & \rho_{L-2} \\
\rho_{2} & \rho_{1} & \rho_{0}+N_{0} & \cdots & \rho_{L-3} \\
\cdot & \cdot & \cdot & & \cdot \\
\cdot & \cdot & \cdot & & \cdot \\
\cdot & \cdot & \cdot & & \cdot \\
\rho_{L-1} & \rho_{L-2} & \rho_{L-3} & \cdots & \rho_{0}+N_{0}
\end{array}\right]\left[\begin{array}{c}
a_{1} \\
a_{2} \\
a_{3} \\
\cdot \\
\cdot \\
\cdot \\
a_{L}
\end{array}\right]=\left[\begin{array}{c}
\rho_{1} \\
\rho_{2} \\
\rho_{3} \\
\cdot \\
\cdot \\
\rho_{L}
\end{array}\right]
$$

where $N_{0}$ is the sing'e-sided additive white gaussian noise density, and the $\left\{a_{1}\right\}$ ar ie predictor coefficients. Note that by normalizing the autocorrelations. we are assuming that the energy per symbol. $E_{\mathrm{s}}$. is $\mathrm{I}$ and so the value of $N_{0}$ is easily determined from the $E_{s} / N_{0}$ required for the simulation.

Solving (6.6) for the $\left\{a_{i}\right\}$, gives us the $L$ coefficients for our predictor

$$
\hat{s}(n)=-\sum_{i=1}^{L} a_{i} s(n-i)
$$

We can transform this into a prediction error filter 


$$
\begin{aligned}
s(n)-\hat{s}(n) & =s(n)-\left[-\sum_{i=1}^{L} a_{i} s(n-i)\right] \\
& =\sum_{i=0}^{L} a_{i} s(n-i)
\end{aligned}
$$

where $a_{0}=1$. For the MLSE and MAP demodulators. $s(n)$ will be the received sample at time $n$ with the hypothesized modulation removed.

Figure 6-10 illustrates the fit of a Sth order predictor for a channel with a single-sided fading bandwidth of $0.3 R$ (at $r=2$ samples/symbol) for $E_{s} / N_{0}$ 's of $5 \mathrm{~dB}$ (Figure 6-10a) and $30 \mathrm{~dB}$ (Figure 6-10b). It is instructive to note that at $5 \mathrm{~dB}$, where the noise dominates receiver performance, the predictor is closely matched to the noise floor. and less to the spectrum of the fading process. Conversely at $30 \mathrm{~dB}$, where the fading dominates receiver performance, the fit to the spectrum of the fading process is improved at the expense of ripple in the soise approximation. This makes intuitive sense since the predictor is generated by minimizing the mean squared prediction error, a process which weights higher power components more strongly. 


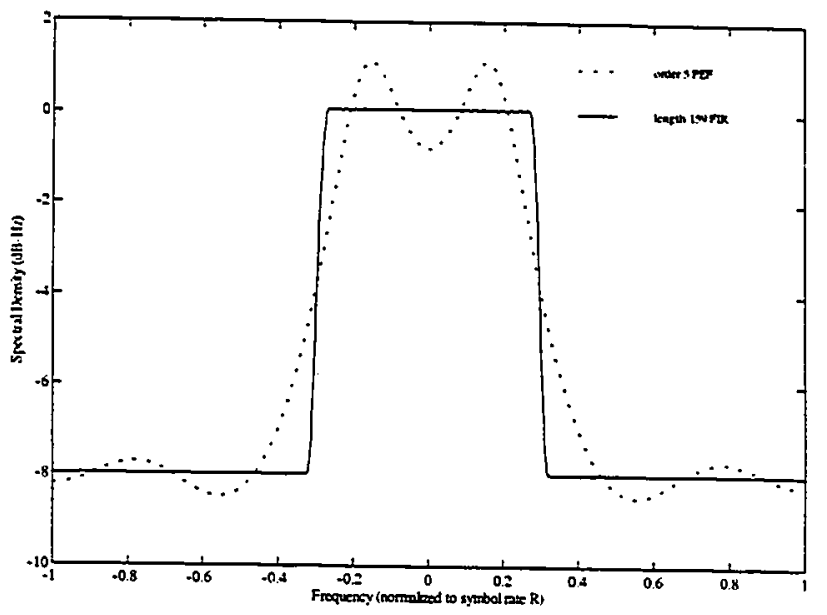

(a) $E_{s} / N_{0}$ of $5 \mathrm{~dB}$

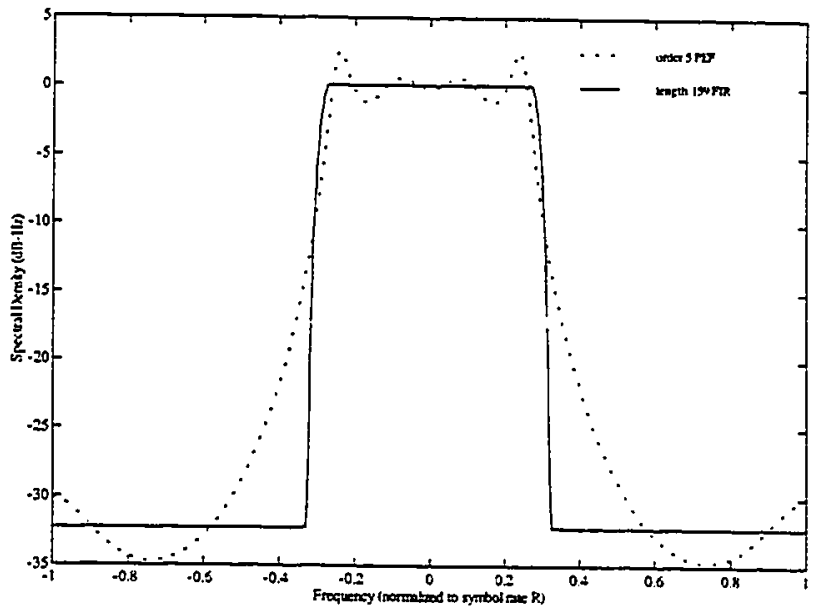

(b) $E_{s} / N_{0}$ of $30 \mathrm{~dB}$

Figure 6-10 Predicted and actual filters for a fading rate of $0.3 R$ at $r=2$ samples/symbol. 
Figure 6-11 illustrates the effect of predictor order on fit for the same fading rate and an $E_{s} / N_{0}$ of $10 \mathrm{~dB}$. It can be seen that even these low order predictors result in good approximations to the composite fading plus noise spectrum. The effect of predictor order can be evaluated quantitatively as discussed in Section 2.3. Figure 6-12 shows the expected normalized prediction errors for the scenario of Figure 6-11.

The choice of predictor order is directly related to the number of states required by the MLSE and MAP demodulators (see Appendix A - CPM Matched Filter Requirements). It is desirable to use the smallest order predictor which provides adequate performance.

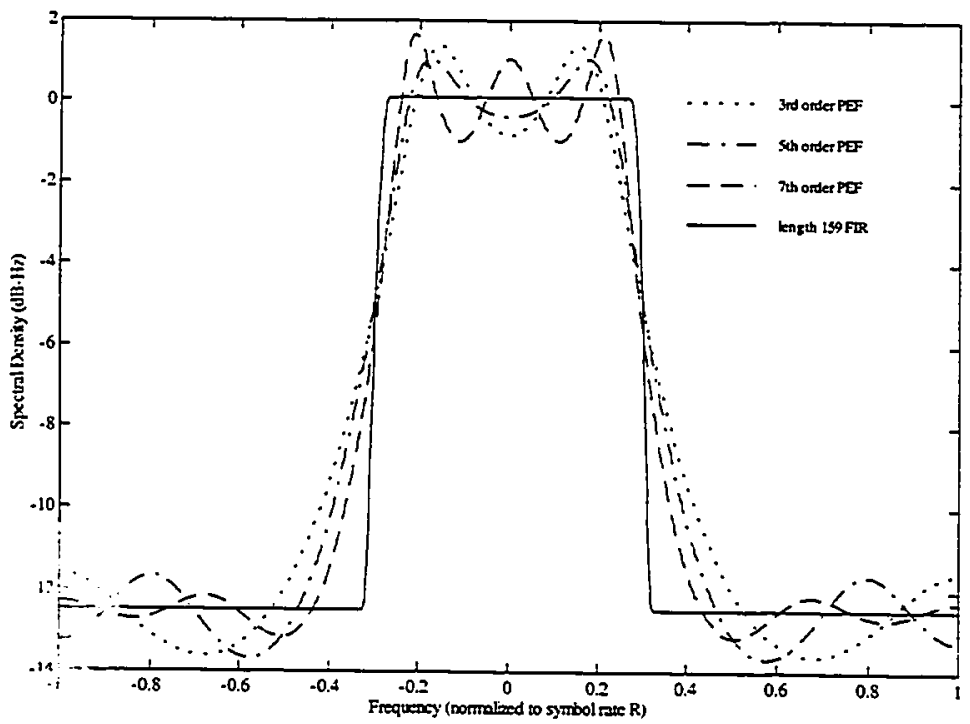

Figure 6-11 The effect of predictor order on fit for a fading rate of $0.3 R$ and an $E_{s} / N_{0}$ of $10 \mathrm{~dB}$ at $r=2$ samples'symbol. 


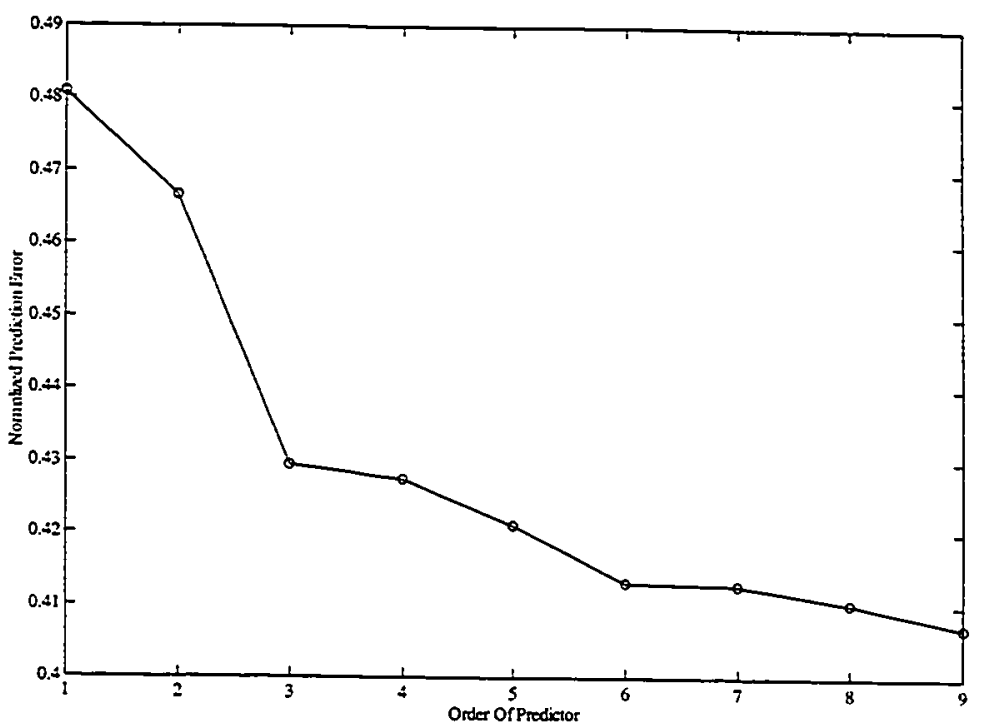

Figure 6-12 Prediction error vs. predictor order for a fading rate of $0.3 R$ and an $E_{s} / N_{0}$ of $10 \mathrm{~dB}$ at $r=2$ samples/symbol.

\subsection{Demodulation Of Binary CPM Signals}

\subsubsection{Uncoded BER Results}

Simulations were performed to compare the performance of the MAP demodulator with that of the MLSE-based linear predictive receiver and that of differential detection. when hard decision outputs from the algorithms were considered. Two fading channel rates were investigated, $0.05 R$ and $0.3 R$. The simulation parameters were:

Fading rate:

$0.05 R, 0.3 R$

Sample rate:

$r=2$ samples/symbol

CPM pulse shape: $\quad$ N32FM ( $K=3$ symbol periods)

Predictor order: $\quad 5$ th $\operatorname{order}(\chi=2.5$ symbol periods) 
The number of MAP and MLSE states was $2^{[3-25]-1}=32$. The resulting BER performance is shown in Figures 6-13 and 6-14 respectively.

A number of points can be noted:

- differential detection results in an irreducible error floor that can not be improved upon by increasing the signal to noise ratio;

- differential detection is essentially useless at a fading rate of $0.3 R$;

- the MAP and MLSE algorithms perform approximately the same;

- there is no sign of an error floor in the MAP and MLSE results, even at an $E_{b} / N_{0}$ of $30 \mathrm{~dB}$ :

- the BER estimate produced by the MAP algorithm closely matches the BER measured in the simulation.

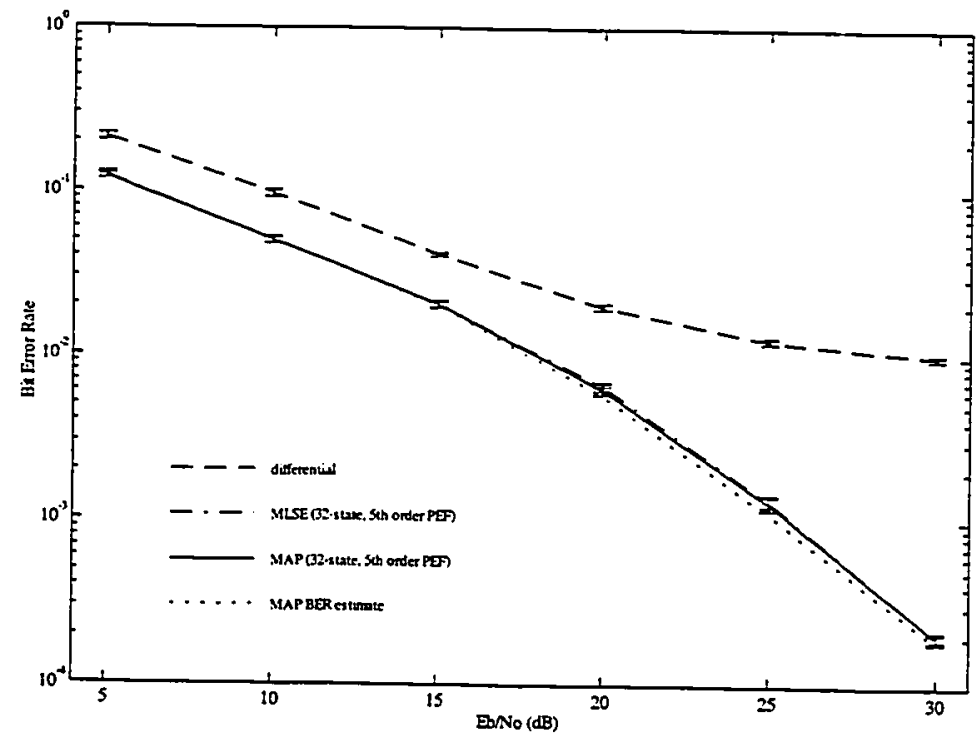

Figure 6-13 The BER performance of the MAP and MLSE demodulators as compared to that of differential detection for the reception of binary CPM signals. The fading rate is $0.05 R$. 


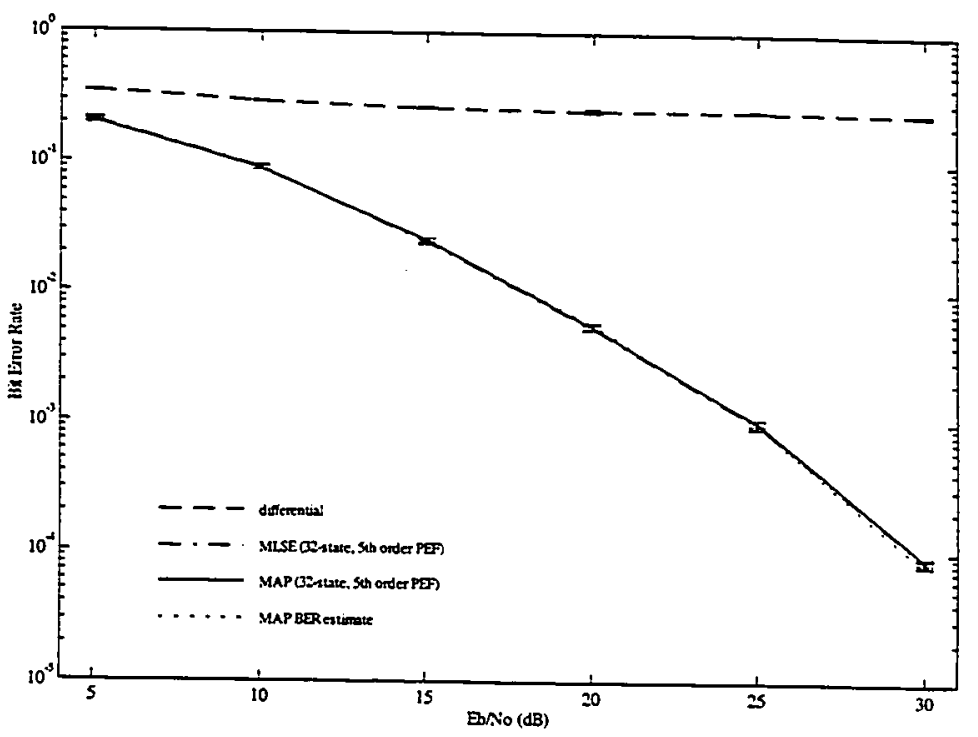

Figure 6-14 The BER performance of the MAP and MLSE demodulators as compared to that of differential detection for the reception of binary CPM signals. The fading rate is $0.3 R$.

The MLSE and differential detection results are consistent with those in [33]. As expected. the difference between MAP and MLSE processing is negligible (given the known performance of the Viterbi algorithm for a sufficient chainback depth).

\subsubsection{BER Estimation Results}

The BER estimates plotted in Figures 6-13 and 6-14 are corrputed by averaging the estimates produced by the MAP algorithm over the whole simulation. As noted above, these estimates are in close agreement with the measured BER. Some divergence is to be expected since the prediction filter is not precisely matched to the composite fading plus noise spectrum, particularly at high signal to noise ratios where the rolioff of the filter must be steep. It is of interest to examine how quickly the BER estimates produced by the algorithm converge to the long term averaged estimate. Consider MAP demodulation 
at an $E_{b} / N_{0}$ of $10 \mathrm{~dB}$ as illustrated in Figure 6-13. The corresponding bit error rate is $5 \times 10^{-2}$. Figure 6-15 illustrates the standard deviation of the BER estimates produced by the algorithm as a function of the number of 1024 bit MAP blocks that the estimate was computed over. It can be seen that the estimate produced by the MAP processing converges to a highly reliable value within just a few thousand bits. For the case where the model is approximately matched to the channel. the estimate appears to be unbiased. A run of 10000 blocks produced an averaged BER estimate of $5.04 \times 10^{-2}$ while a bit error measurement for the same run found a BER of $5.05 \times 10^{-2}$. Note that due to the correlated ature of the bit errors. the convergence time is a strong function of the expected bit error rate.

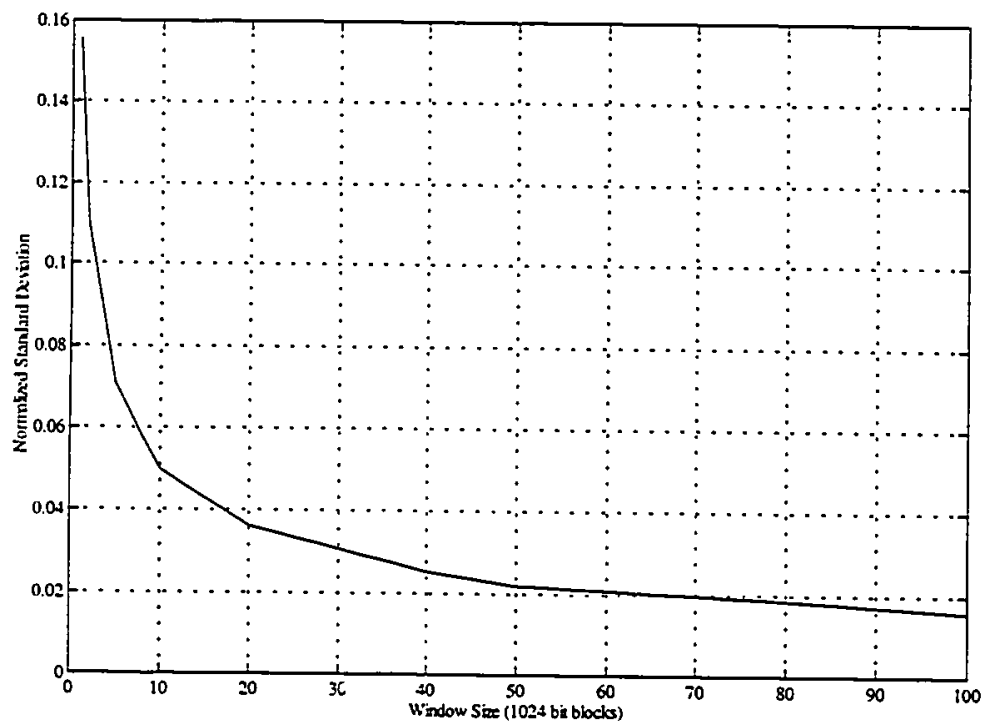

Figure 6-15 The BER estimation performance of the MAP demodulator for the reception of binary CPM signals as a function of window size. The fading rate is $0.05 R$ and the $E_{b} / N_{0}$ is $10 \mathrm{~dB}$. 


\subsubsection{Coded BER Results}

In order to evaluate the effectiveness of the soft decisions produced by the symbol-bysymbol MAP demodulator, the output probabilities from the demodulator were fed to a following MAP convolutional decoder and the resulting BER evaluated. A MAP decoder is utilized, rather than a decoder utilizing the Viterbi algorithm. since it inherently operates on probabilities.

The code utilized is the industry standard rate-1/2, constraint length 7 convolutional code [35]. A 128 row $\times 8$ column interleaver is used to decorrelate the bit errors prior to decoding. While this interleaver does not represent ideal interleaving. it does provide most of the associated gain while remaining a practical length.

In Figures 6-16 and 6-17, the performance of MAP demodulation is compared with that of soft decision decoding of differentially detected CPM. A 16-state MAP demodulator is used since truncating the pulse shape by one sampie has only a negligible effect. As a reference. the performance of hard decision decoding using the outputs of the linear predictive receiver is also presented. From Figure 6-16. a number of points are apparent:

- the simple soft decisions from a differential detector work quite well at a fading rate of $0.05 R$ :

- the improved soft decisions from the MAP demodulator provide over $4 \mathrm{~dB}$ of additional gain at an error rate of $10^{-5}$; and.

- the soft decisions from the MAP demodulator provide over $7 \mathrm{~dB}$ of gain relative to the hard decisions from the MLSE demodulator. 


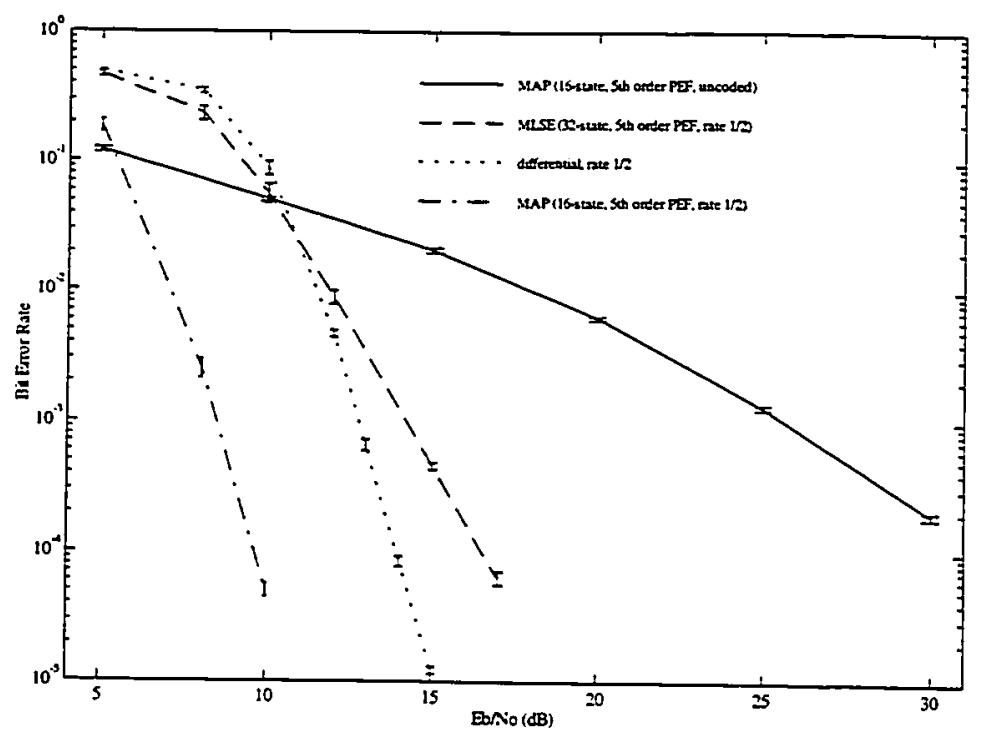

Figure 6-16 The coded and uncoded BER performance of the MAP and MLSE demodulators as compared to that of differential detection for the reception of binary CPM signals. The fading rate is $0.05 R$. Coded MLSE results utilize hard decision inputs to the decoder while the coded MAP and differential detection results utilize soft decisions.

When the fast fading channel of Figure 6-17 is examined. we can see that:

- the soft decisions from the differential detector are no longer capable of driving the Viterbi decoder:

- the soft decisions from the MAP demodulator continue to provide good performance, exhibiting approximately $5 \mathrm{~dB}$ of gain relative to the hard decisions from the MLSE demodulator at an error rate of $10^{-5}$. 


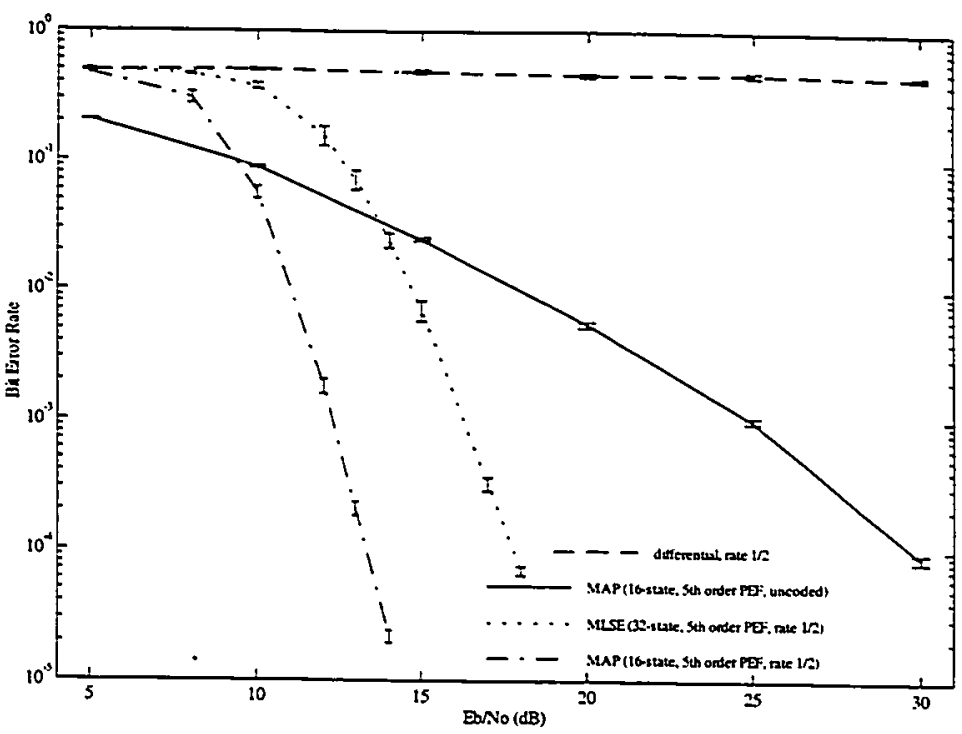

Figure 6-17 The coded and uncoded BER performance of the MAP and MLSE demodulators as compared to that of differential detection for the reception of binary $\mathrm{CPM}$ signals. The fading rate is $0.3 R$. Coded MLSE results utilize hard decision inputs to the decoder while the coded MAP and differential detection results utilize soft decisions.

\subsubsection{Average Mutual Information}

In Section 6.2.3 the performance of a soft decision convolutional decoder following the demodulator was used to compare the quality of the soft decisions out of the MAP demodulator with that of the soft decisions from the differential detector. Here, we perform the same type of comparison using average mutual information as a metric.

The average murual information between two events refers to the amount of information, that the occurrence of one event provides about the other. In this section. we are interested in the information that the output of the demodulator (which can be hard or soft decisions) provides about what was input to the modulator. 
In general, for any two discrete random variables $W$ and $Z$, with possible outcomes $\left\{w_{i}\right\}$, $1 \leq i \leq i^{\prime}$ and $\{z j\}, 1 \leq j \leq j^{\prime}$ respectively, their average mutual information is given by [21. eqn. 2.2.7]:

$$
I(W ; Z)=\sum_{i=1}^{\dot{C}} \sum_{j=1}^{\dot{1}} P\left(w_{i}, z_{j}\right) \log \frac{P\left(w_{i}, z_{j}\right)}{P\left(w_{i}\right) P\left(z_{j}\right)}
$$

For the case of hard decision output from a demoduiator, the $\left\{u_{i}\right\}$ and $\left\{z_{j}\right\}$ are bits which can take on values of 0 or 1 giving

$$
I_{h d}(W ; Z)=\sum_{i=0}^{1} \sum_{j=0}^{1} P(W=i, Z=j) \log \frac{P(W=i . Z=j)}{P(W=i) P(Z=j)}
$$

where $W$ represents the input bits and $Z$ represents the hard decisions out of the demodulator. If a base 2 logarithm is used, the resulting quantity is in units of bits.

For the case of soft decisions out of the demodulator, the input random variable is discrete while the output is continuous. The average mutual information is then given by [21]:

$$
\begin{aligned}
I_{x}(W: Z) & =\sum_{i=0}^{1} \int_{-} p\left(z \mid w_{i}\right) P\left(w_{i}\right) \log \frac{p\left(z \mid w_{i}\right)}{p(z)} \\
& =\sum_{i=0}^{1} \int_{-} p(z \mid W=i) P(W=i) \log \frac{p(z \mid W=i)}{p(z)}
\end{aligned}
$$

Both (6.10) and (6.11) were evaluated numerically over samples of approximately 240.000 bits for both differential detection and symbol-by-symbol MAP demodulation of binary CPM. In the case of the differential detector. the soft decision was deemed to be $\operatorname{Im}\left\{y, y_{j-1}^{*}\right\}$ where $y_{j}$ is the received sample for the $j$ th symbol. For the MAP demodulator, the soft decision was simply the bit probability per (6.3). The results are shown in Figures 6-18(a) through 6-18(d). 


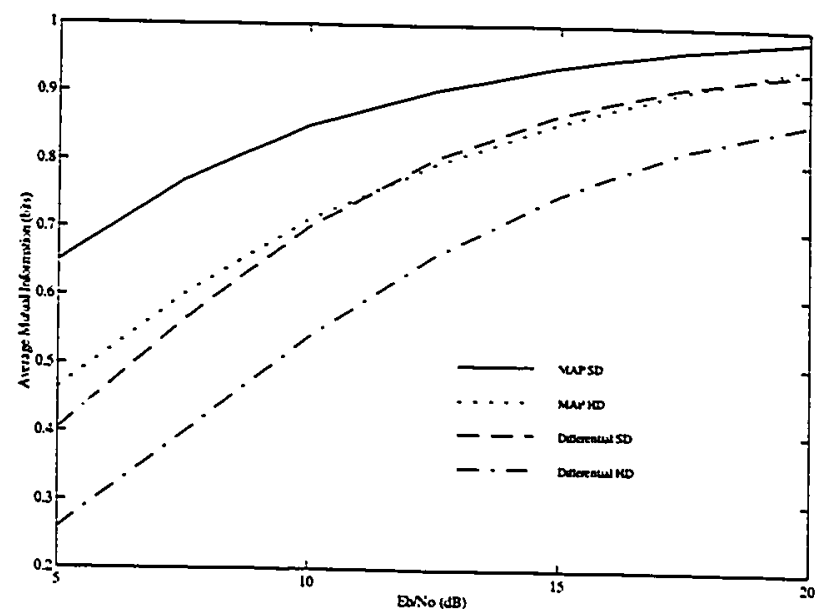

(a) Soft and hard decision quality for a fading rate of $0.05 R$

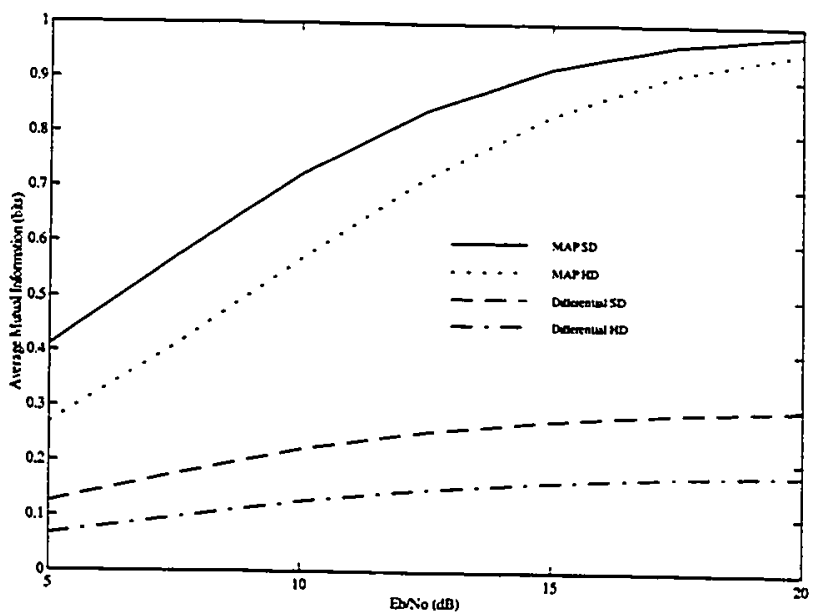

(b) Soft and hard decision quality for a fading rate of $0.3 R$ 


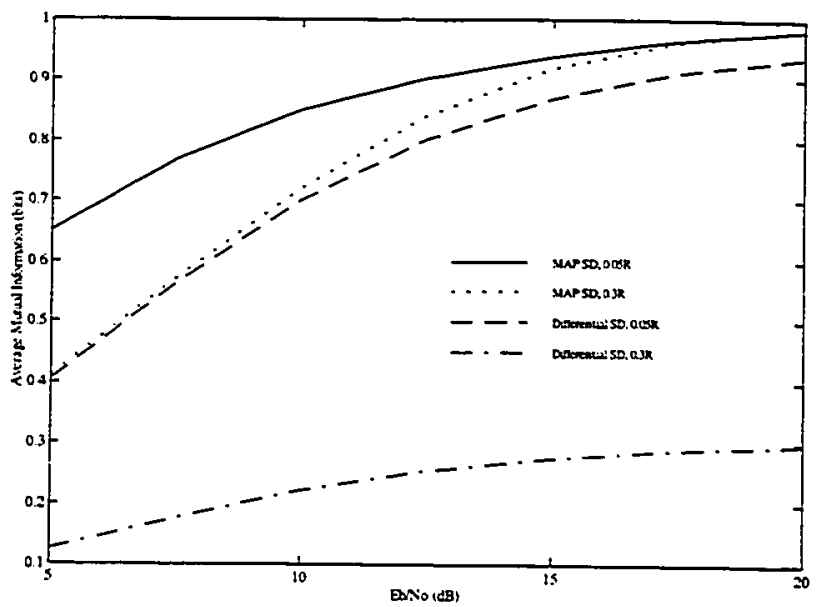

(c) Soft decision quality for different fading rates

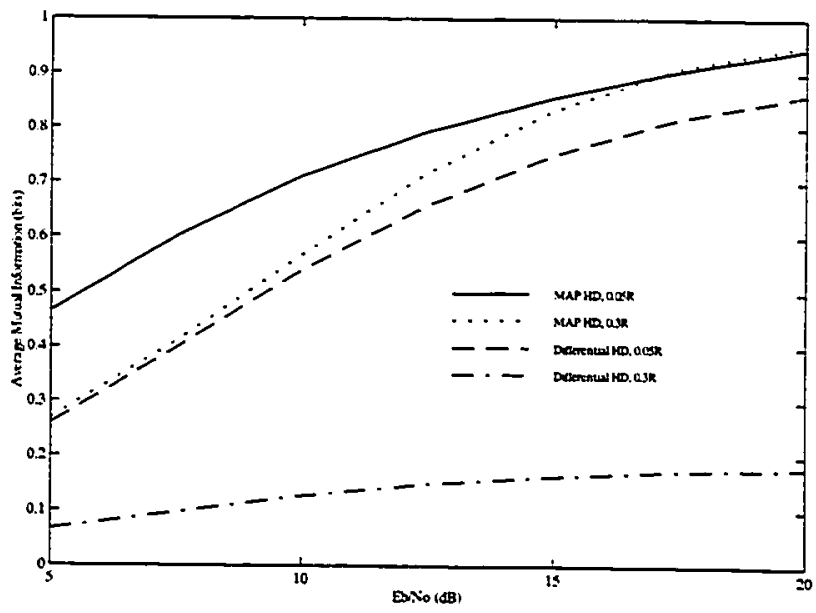

(d) Hard decision quality for different fading rates

Figure 6-1S Comparisons of hard and soft decision quality. 
A number of points can be noted:

- except at high $E_{b} / N_{0}$. the hard/soft decisions for both differential detection and MAP demodulation have higher average mutual information on the slower fading $0.05 R$ channel:

- at high $E_{b} / N_{0}$, the hard/soft decisions for MAP demodulation have slightly higher average mutual information on the faster fading $0.3 R$ channel. likely due to the greater diversity within a symbol period that is present on that channel:

- the results for the $0.05 R$ channel are consistent with those of Figure 6-16. with the interesting result that the point at which the murnal information in the

MAPMLSE hard decisions matches that of the differential detection sott decisions. i.e.. an $E_{b} / N_{0}$ of approximately $12 \mathrm{~dB}$ (see Figure 6-1S(a)), is the same point at which the two yield the same performance in a rate-1/2 coded system:

- similarly. the results for the $0.3 R$ channel are consistent with those of Figure 6-17.

In all cases, the superiority of the hard and soft decisions produced by symbol-by-symbol MAP demodulation over those produced by differential detection is clearly evident.

\subsubsection{Reduced State Performance}

Given the computational complexity of the symbol-by-symbol MAP algorithm, it is highly desirable to minimize the number of states required. In this section. two approaches are examined:

- using a lower order predictor: and/or.

- using a truncated form of the transmitied pulse shape when removing the hypothesized modulation.

It is also of interest to determine whether increasing the predictor order. without increasing the number of states. has a beneficial effect on performance.

Table 6-1 summarizes the configurations over which the uncoded BER performance was compared. 


\begin{tabular}{|c|c|c|}
\hline $\begin{array}{c}\text { Pulse Lengh } \\
\text { K (symtwis) }\end{array}$ & $\begin{array}{c}\text { Predictor Order } \\
2 \chi \text { (samples) }\end{array}$ & $\begin{array}{c}\text { Number Of } \\
\text { Stzies Lised }\end{array}$ \\
\hline 1.5 & 3 & 4 \\
\hline 2.5 & 3 & $s$ \\
\hline 1.5 & 5 & 5 \\
\hline 2.5 & 5 & 16 \\
\hline 2.5 & 7 & 32 \\
\hline
\end{tabular}

Table 6-1 The set of reduced state test configurations.

Figures 6-19 and 6-20 illustrate the effect of the above configurations on the performance of binary CPM for fading rates of $0.05 R$ and $0.3 R$ respectively.

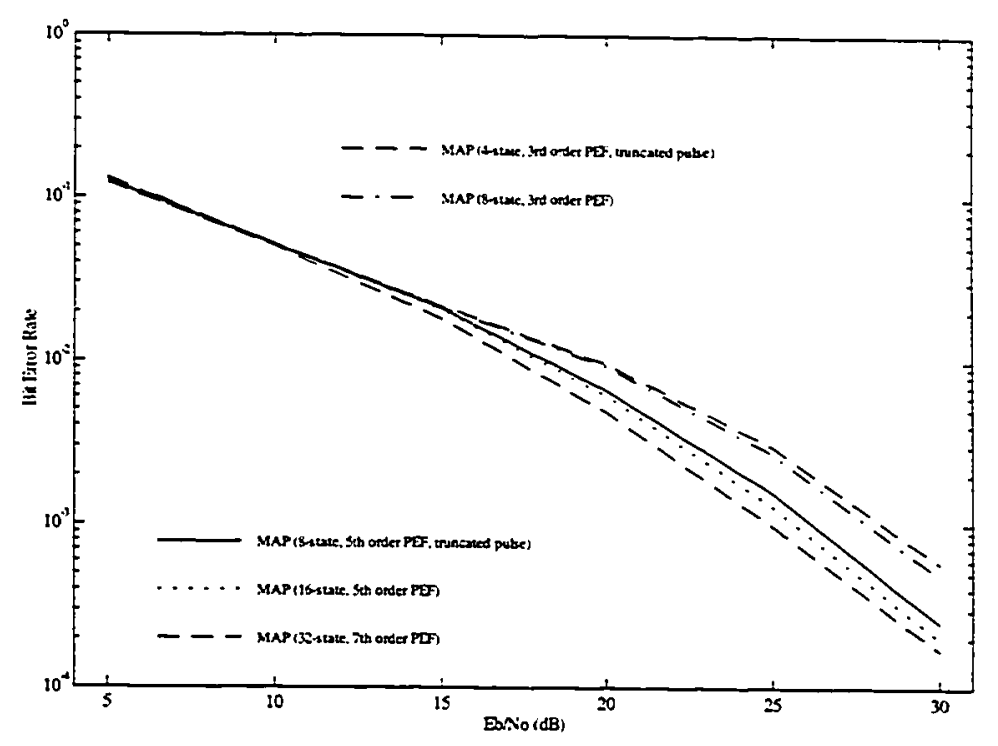

Figure 6-19 The reduced-state BER performance of the MAP demodulator. The fading rate is $0.05 R$. 


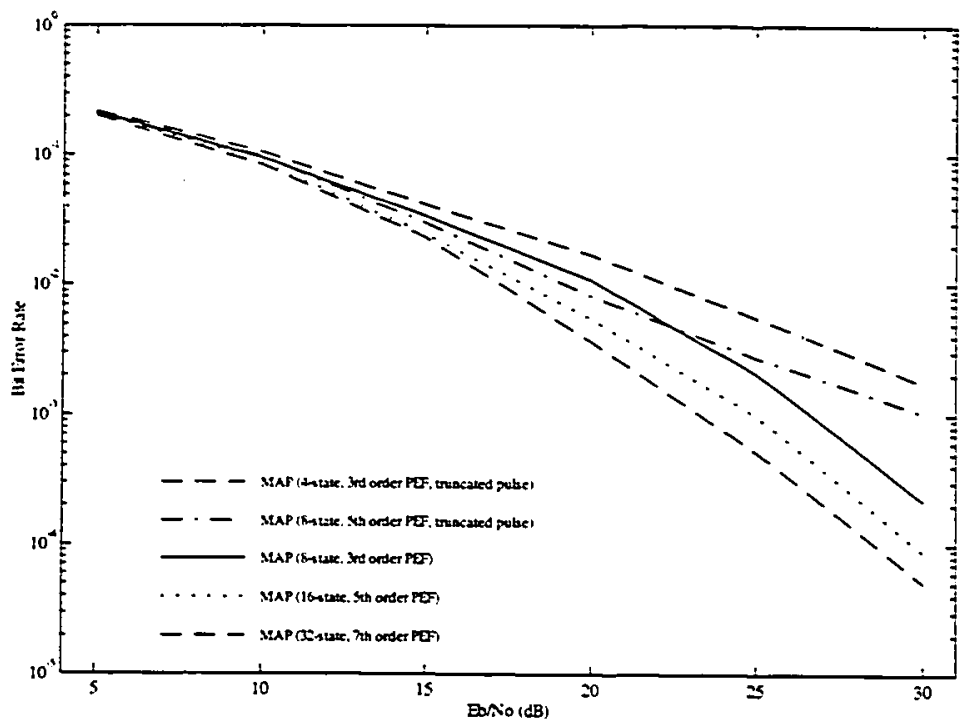

Figure 6-20 The reduced-state BER periormance of the MAP demoduiator. The fading rate is $0.3 R$.

A number of resuits can be observed:

- as expected. configurations utilizing a larger number of states provide better performance:

- increasing the number of states has a greater effect at the higher fading rate:

- there is no clear conclusion which can be drawn regarding the tradeoff between reducing the number of states by truncaring the pulse shape versus achieving the same complexity by reducing the predictor order. truneating the pulse shape worked best at the lower fading rate while decreasing the predictor order worked best at the higher fading rate.

Further insight into the effect of reduced state implementations on the soft decisions produced by the MAP demodulator can be gained by evaluating the performance of the 
MAP demodulator's BER estimator. For each of the contigurations of Table 6-1, the BER estimate produced by the M.AP demodulator closely matched the measured BER. This implies that while reduced state implementations suffer from degraded performance. the conditions required for the symbol-by-symbol MAP processing to produce optimal. or near-optimal. probability estimates are still satisfied.

\subsection{Sensitivity To Channel Model}

One of the key inputs to the symbol-by-symbol MAP demodulator is the channel model as represented by the prediction error filter. It is of interest to determine the sensitivity of the algorithm to errors in this predictor. Two types of errors are investigated - crors in the estimated noise floor. and errors in the estimated bandwidth of the fading process.

Figure 6-21 illustrates the sensitivity of the demodulator to an etror in the modelled noise level for a fading rate of $0.05 R$ and an $E_{b} / N_{0}$ of $10 \mathrm{~dB}$. These parameters represent the situation where the performance of the receiver is dominated by the effect of the noise. From the figure, we can see that the uncoded BER performance of the demodulator is largely unaffected when the noise level of the model is even $10 \mathrm{~dB}$ higher than the actual noise level. When the modelled noise level is less than the actual noise level. some degradation in performance is observed. although the total effect is only approximately $0.7 \mathrm{~dB}$ with a noise level error of $10 \mathrm{~dB}$ and is insignificant over an error range of $\pm 3 \mathrm{~dB}$. With knowledge of the actual prediction error, the MAP algorithm's BER estimate is only slightly more sensitive to large errors in the modelled noise level. with a degradation of approximately $1.7 \mathrm{~dB}$ observed at a noise level error of $10 \mathrm{~dB}$. However, with a prediction error estimated solely from the measured autoconrelation. the BER estimatc was very sensitive to noise level error.

The flatness of the BER performance results is not surprising. Since prediction errors due to noise are independent of the hypothesized sequence. they can be expected to be fairly evenly distributed across all of the hypotheses. The greater sensitivity of the BER estimation is also not unexpected, since the expected prediction error is used to weight the inputs to the MAP processing (see Section 6.2.7 below). 


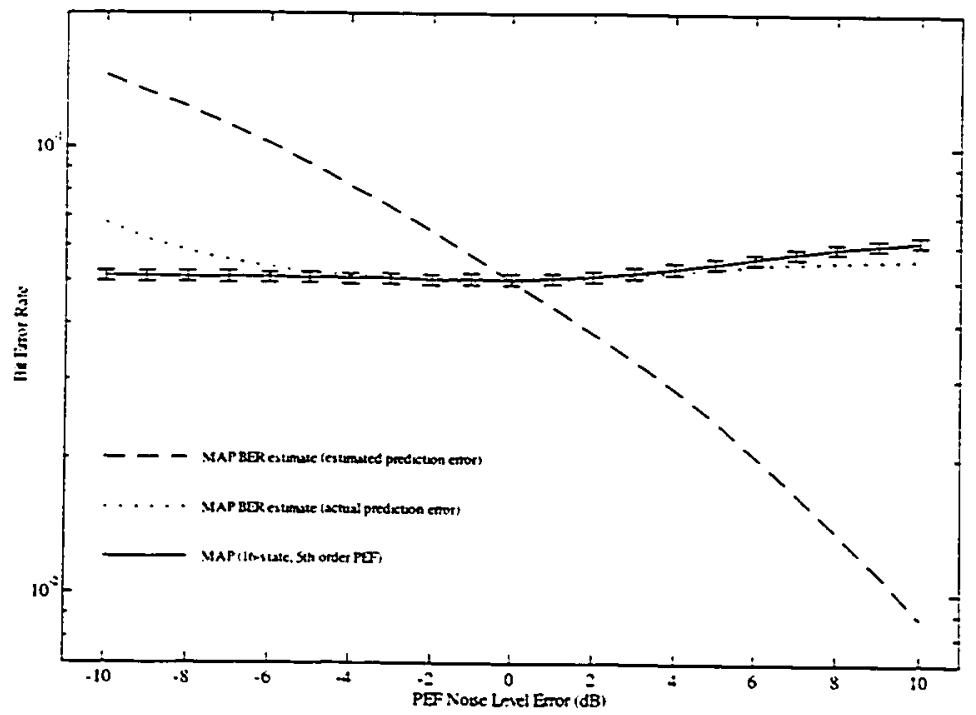

Figure 6-21 The sensitivity of the binary CPM BER performance of the MAP demodulator to errors in the modelled AWGN noise level. Tne $E_{b} / N_{0}$ is $10 \mathrm{~dB}$. representing the case where the effect of the noise dominates the performance. The fading rate is $0.05 R$.

Figure 6-22 illustrates the sensitivity of the demodulator to an error in the modelled fading bandwidth for a fading rate of $0.3 R$ and an $E_{b} / N_{0}$ of $25 \mathrm{~dB}$. These parameters represent the situation where the performance of the receiver is dominated by the effect of the fading. From the figure. we can see that the BER performance is clearly affected by errors in the model. Errors introduced when the modelled bandwidth is less than the actual bandwidth are greater than those introduced when the fading bandwidth is overestimated. although the difference is only substantial when the error is over $20 \%$. An interesting result is that the BER estimator remains fairly accurate at predicting the BER in the presence of an error in the modelled fading bandwidth. if the prediction error used by the MAP processing is the true prediction error for each block. If the expected squared 


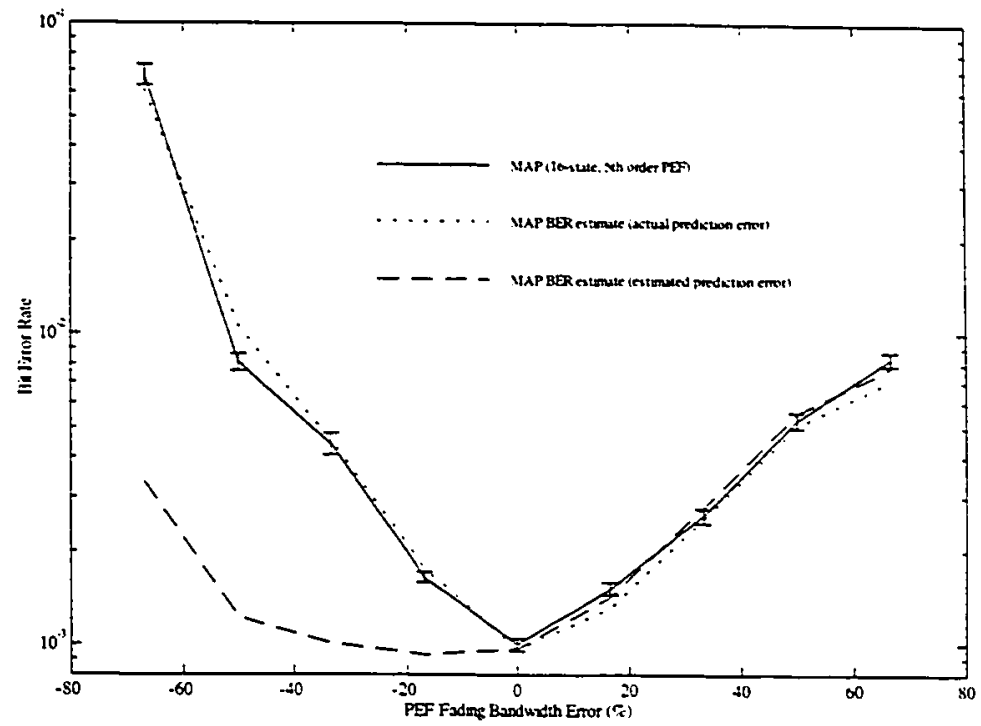

Figure 6-22 The sensitivity of the binary CPM BER performance of the MAP demodulator to errors in the modelled fading bandwidth. The $E_{b} / N_{0}$ is $25 \mathrm{~dB}$. representing the case where the effect of the fading dominates the performance. The fading rate is $0.3 R$.

prediction error is used instead. the BER estimates are biased, although only for the case where the bandwidth of the fading process was underestimated.

\subsubsection{Sensitivity To Expected Prediction Error}

Recall that the outputs of the prediction etror fitters for each hypothesis are divided by the expected squared prediction error. $r_{0} V_{L}$, before the exponential is taken per (5.22). i.c..

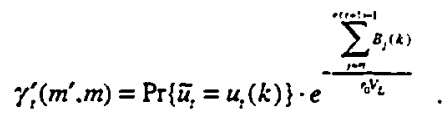


Since the identical division is performed for each hypothesis. it has no effect on the relative weighting of different hypotheses (in fact in the MLSE-based linear predictive receiver of [33]. this term is ignored completely since it is a common factor in all metries being fed to the Viterbi algorithm). Notwithstanding this, the expected squared prediction ertor plays an important role in determining the probability censity of the demodulated soft decisions. In essence. it provides reliability information about the predictor which is inherently utilized to weight the symbol probabilities. For example. consider the determination of the symbol probability for a symbol. $u_{t}$. which is strongly a "1". The determined symbol probability, $P\left(u_{1}=1\right)$, will be higher for a low expected squared prediction error than for a high expected squared prediction error.

In general. the impact of an incorrect $r_{0} V_{L}$ will be minimal on a system which utilizes the symbol probability to make hard decisions. This is because its effect will be symmetrical for both 1 "s and 0 's. In contrast. errors in $r_{0} V_{L}$ may have a large impact on the soft decisions (and therefore BER estimates) output by the demodulator.

A number of simulations were performed to investigate the impact of errors in the computation of the expected squared prediction ertor on the performance of the demodulator. Three mechanisms for determining $r_{0} V_{L}$ were implemented:

a) computation of the actual squared prediction error for each block by stripping off the modulation using the actual values of the transmitted bits (a mechanism which an actual receiver could obviously not use) and then running the resulting samples through the prediction error filter:

b) computation of the zero-lag autocorrelation. $r_{0}$. for each block and then multiplying this by expected normalized squared prediction error. $V_{L}$. (which can be computed directly from the coefficients of the linear predictor): and.

c) explicitly specifying $r_{0} V_{L}$.

For the cases where the PEF was matched to the channel (within the limitations of the predicto: order). mechanisms (a) and (b) above produced essentially the same hard decision. soft decision and BER estimation performance. As was seen in Figure 6-22. this does not hold fsi the case of errors in the model. Figure 6-23 illustrates the variation 


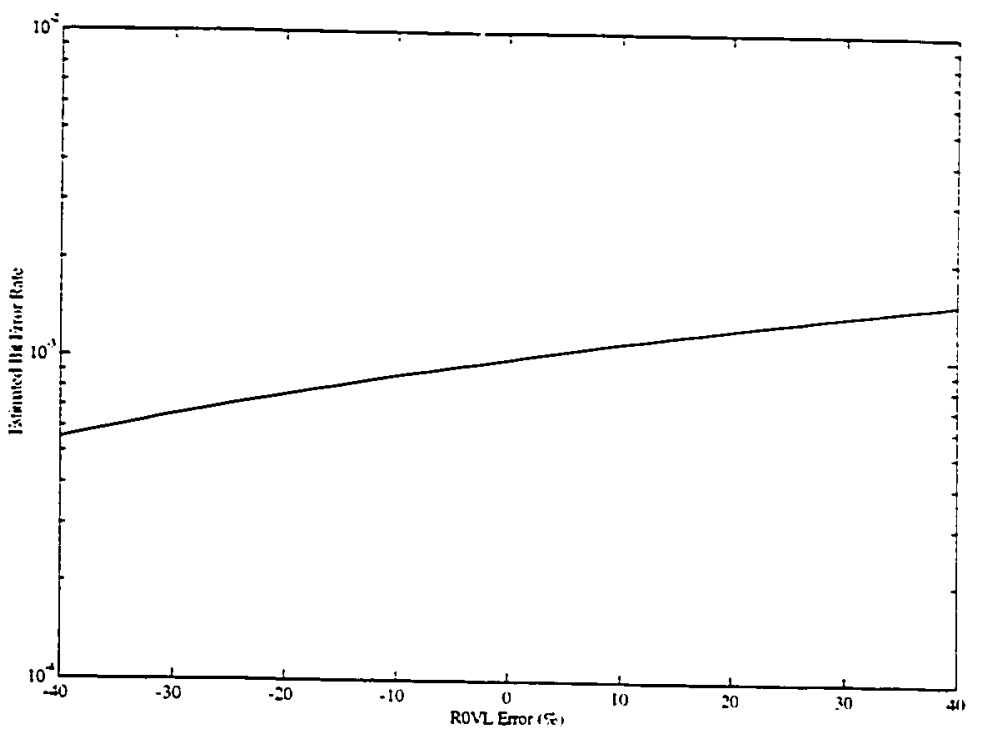

Figure 6-23 The sensitivity of the binary CPM BER performance of the MAP demodulator to errors in the estimared squared prediction ertor $\left(r_{0} V_{L}\right)$. The $E_{h} / N_{0}$ is $25 \mathrm{~dB}$ and the fading rate is $0.3 R$.

of the estimated BER as a function of the error in computing $r_{0} V_{L}$. As might be expected. when the expected squared prediction error is underestimated. the symbol probabilities are overly optimistic while the converse is true when the prediction error is overestimated.

If errors in the model are found to be a problem. one means of ensuring that an accurate prediction efror is used would be to use the hard decisions output by the demodulator to provide a new estimate for the squared prediction ertor using mechanism (a) above.

Averaging could be used to damp transients due to demodulation errors since the expected error does not change significantly from block to block unless the channel is changing also. 


\subsection{Demodulation Of QPSK Signals}

In this section we consider the performance of symbol-by-symbol MAAP demodulation of QPSK signals. At I sample per symbol. QPSK can be considered to be a discrete time form of CPM.

The performance for QPSK is only evaluated at the fading rate of $0.05 R$. At this rate, it is assumed that the effect of receiver matched filtering on the constellation of the faded QPSK is minimal. If higher fading rates were to be considered. a realistic simulation would have to take into account the spreading of the constellation caused by receive filtering.

\subsubsection{Phase Slips}

From the receiver structure of Figure 5-4, we can see that the inputs to the MAP algorithm involve the squared magnitudes of the filier bank outputs. A consequence of this is that there is a four-fold phase ambiguity on the branch updates. While this means that we can reduce the number of filters in the filter bank by a factor of four. it unfortunately aiso means that the only mechanism that the MAP algorithm has for resolving the ambiguivies is the initial and final states. and the structure of the trellis (which is highly connected). As a result. when the error rate is significant. error events can cause the MAP demodulator to enter the wrong state. and therefore consistently choose symbols which are $90^{\circ}$ (or less likely. $180^{\circ}$ ) away from the original. The demodulator will remain in this state until either some event causes it to retum to the proper state, or the information in the trellis forces it to the proper state.

There are a number of common approaches to combat this problem. The first is to differentially encode the signal so that the absolute phase of the received signal is not important. only the phase difference between symbols. This approach works extremely well. but in a Rayleigh fading channel it introduces a $3 \mathrm{~dB}$ penaly when compared with coherent detection [1S]. An altemative approach is to include known pilot symbols in the transmitted symbol stream as is done in PS.AM systems. The difference here is that the pilot symbols need not be at a rate high enough to sample the fading: they are only 
included to resoive phase ambiguities. This will reduce the bandwidth penalty which is incurred by the pilot symbols.

A number of simulations were performed to evaluate the optimum pilot symbol spacing. As would be expected. higher pilot symbol rates are required at lower signal to noise ratios. Table 6-2 illustrates some approximate uncoded bit error probabilities for an $E_{b} / N_{0}$ of $10 \mathrm{~dB}$ and a number of pilot symbol rates.

\begin{tabular}{|c|c|c|}
\hline Pilot Symbol Rate & Bit Error Rate & Bandwidth Penalty \\
\hline $1: 4$ & $3.95 \times 10^{2}$ & $25 \%$ \\
\hline $1: 8$ & $4.15 \times 10^{2}$ & $12.5 \%$ \\
\hline $1: 16$ & $7.96 \times 10^{\circ}$ & $6.25 \%$ \\
\hline $1: 32$ & $2.04 \times 10^{-1}$ & $3.125 \%$ \\
\hline $1: 64$ & $3.15 \times 10^{-1}$ & $1.5625 \%$ \\
\hline
\end{tabular}

Table 6-2 Performance Versus Pilor Symbol Rate At $E_{b} / N_{0}=10 \mathrm{~dB}$

As is evident from the table. at this low SNR. even pilot symbol rates as high as 1 in 5 continue to improve the performance of the demodulator. albeit at the expense of up to $25 \%$ bandwidth overhead. As a compromise between performance and bandwidth overhead. pilot symbol rates of $I$ in $9(1: \$)$ and $I$ in $17(1: 16)$ were chosen for further study.

\subsubsection{Uncoded BER Results}

Simulations were performed to compare the performance of the MAP demodulator with that of differential detection of differentially encoded QPSK. when hard decision outputs from the algorithms were considered. The simulation parameters were:
Fading rare:
$0.05 R$
Sample rate:
$r=$ I sample/symbol
Symbol encoding:
Gray coding
QPSK pulse shaping:
none ( $\kappa=1$ symbol period) 
MAP predictor order: 3 rd order $(\chi=3$ symbol periods)

MAP pilot symbol rate: $\quad I: S, 1: 16$

The number of MAP states was $4^{-1-3 i-1}=64$. The resulting BER performance is shown in Figure 6-24.

A number of points can be noted:

- as with the binary CPM case. differential detection results in an irreducible error floor that can not be improved upon by increasing the signal to noise ratio:

- at $E_{b} / N_{0}$ of less than $20 \mathrm{~dB}$. the higher pilot symbol rate provides improved MAP demodulator performance;

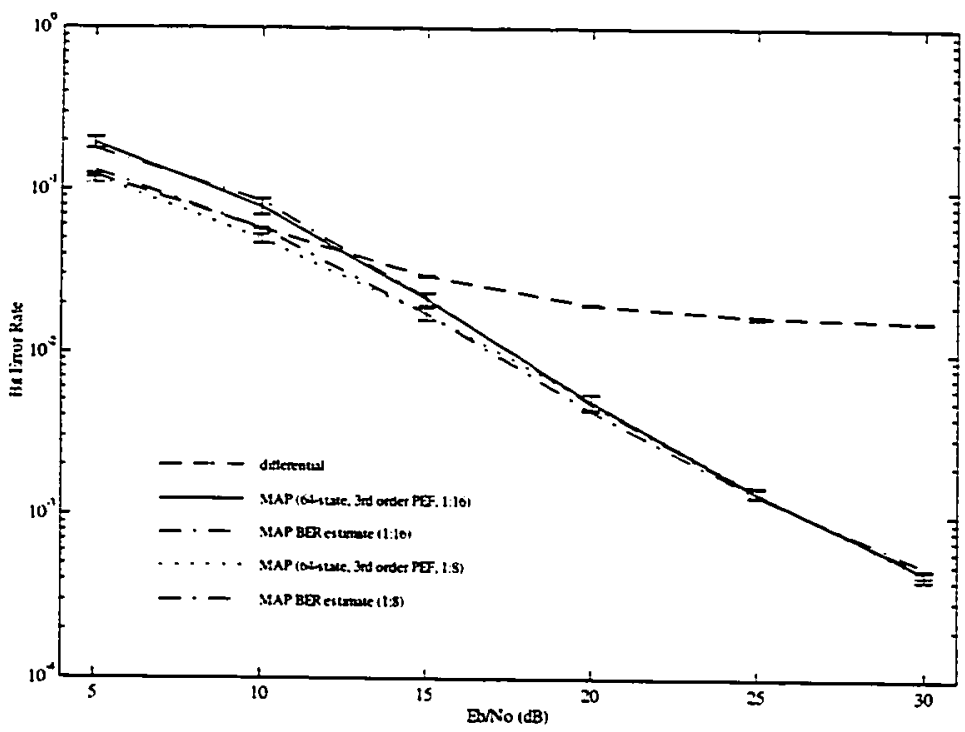

Figure 6-24 The BER performance of the MAP demodulator as compared to that of differential detection for the reception of QPSK signals. The fading rate is $0.05 R$. For the MAP simulations. pilot symbol rates of $1: S$ and $1: 16$ were used. For the differential detection simulations. the signal was differentially encoded. 
- there is no sign of an error floor in the MLAP results. even at an $E_{N} / N_{0}$ of $30 \mathrm{dS}$ :

- the BER escimates produced by the MAP algorithm closely match the BER's measured in the simulation.

The error bursts produced by the MAP demodulator are longer in duration than those obtained using differential detection. This suggests that the M.AP ertor bursts are not just caused by the channel entering a deep fade but are instead caused by phase slips. The resulting error runs dominate the bit error rate. Note that the length of an error run due to a phase slip can exceed the pilot symbol spacing since the pilot symbol only reduces the number of possible states from 64 to 16 . Three contiguous pilot symbols would be required to guarantee resolution of the phase ambiguity every pilot symbol period.

The narure of the error runs was confirmed by examining the symbol probabilities output by the MAP demodulator. Two characteristics of a large number of the error runs were that:

- the distribution of the probabilities remained essentially constant over the run: and.

- the distribution of the probabilities, while constant. was rarely dominated by a single symbol probability.

This latter characteristic is counter to what one would expect from a normal sequence of near-constant symbol probabilities. Near-constant symbol probabilities are normally associated with a period of relatively high SNR where the probability of the chosen symbol approaches one and the probability of the remaining symbols approach zero. This is in contrast to the sequence of probabilities shown in Table 6-3 which were obtained during a run in which all of the input bits were set to 1 (to aid in identifying bit errors).

Note that for the majority of the error run the symbol probabilities are essentially constant. with no one symbol having a probability which approaches one. The majority of the symbol periods have symbol probabilities close to those shown in Figure 6-25. 


\begin{tabular}{|c|c|c|c|c|c|c|}
\hline $\begin{array}{c}\text { Symbol 0 } \\
\text { Probability }\end{array}$ & $\begin{array}{c}\text { Symbol 1 } \\
\text { Probability }\end{array}$ & $\begin{array}{c}\text { Symbol 2 } \\
\text { Probability }\end{array}$ & $\begin{array}{c}\text { Symbol 3 } \\
\text { Probability }\end{array}$ & $\begin{array}{c}\text { Symbol } \\
\text { HD }\end{array}$ & $\begin{array}{c}\text { Bit \#1 } \\
\text { HD }\end{array}$ & $\begin{array}{c}\text { Bit \#2 } \\
\text { HD }\end{array}$ \\
\hline 0.05 & 0.31 & 0.07 & 0.57 & 3 & 1 & 1 \\
\hline 0.07 & 0.30 & 0.05 & 0.58 & 3 & 1 & 1 \\
\hline 0.14 & 0.39 & 0.02 & 0.45 & 3 & 0 & 1 \\
\hline 0.14 & 0.39 & 0.02 & 0.45 & 3 & 0 & 1 \\
\hline 0.14 & 0.39 & 0.02 & 0.45 & 3 & 0 & 1 \\
\hline 0.14 & 0.39 & 0.02 & 0.45 & 3 & 0 & 1 \\
\hline 0.14 & 0.39 & 0.02 & 0.45 & 3 & 0 & 1 \\
\hline 0.14 & 0.39 & 0.02 & 0.45 & 3 & 0 & 1 \\
\hline 0.14 & 0.39 & 0.02 & 0.45 & 3 & 0 & 1 \\
\hline 0.14 & 0.39 & 0.02 & 0.45 & 3 & 0 & 1 \\
\hline 0.14 & 0.39 & 0.02 & 0.45 & 3 & 0 & 1 \\
\hline 0.14 & 0.39 & 0.02 & 0.45 & 3 & 0 & 1 \\
\hline 0.14 & 0.39 & 0.02 & 0.45 & 3 & 0 & 1 \\
\hline 0.14 & 0.39 & 0.02 & 0.45 & 3 & 0 & 1 \\
\hline 0.02 & 0.51 & 0.01 & 0.46 & 1 & 0 & 1 \\
\hline 0.01 & 0.11 & 0.07 & 0.81 & 3 & 1 & 1 \\
\hline
\end{tabular}

Table 6-3 Symbol Probabilities For Phase Slip Error Run

For this particular error run. the symbol hard decision is in fact correct; however, due to the distribution of the probabilities. one bit error is made on every symbol in the run. The effect of this error on subsequent soft-in processing is mitigated by the fact that the soft decision. i.e.. the probability that the bit is a one. for the bit in error is 0.47 (indicating a 0 with a low degree of confidence) while the soft decision for the correct bit is 0.84 (indicating a I with a greater degree of confidence). 


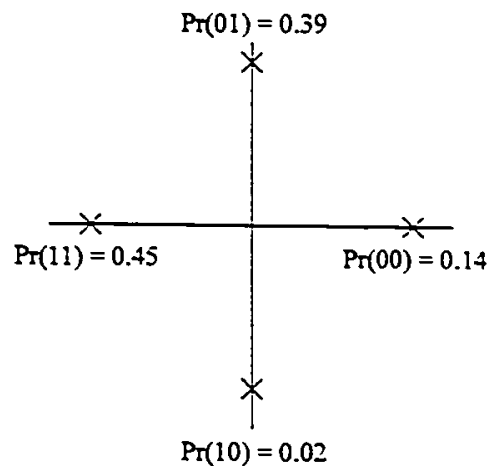

Figure 6-25 An example of the QPSK symbol probabilities after a phase slip has occurred.

\subsubsection{Coded BER Results}

As in the binary CPM case. we wish to evaluate the effectiveness of the soft decisions produced by the symbol-by-symbol MAP demodulator using the BER out of a following MAP convolutional decoder.

The code utilized is the industry standard rate-1/2. constraint length 7 convolutional code [35]. A 128 row $\times 32$ column interleaver is used to decorrelate the bit errors prior to decoding. While this interleaver does not represent ideal interleaving. it does provide most of the associated gain while remaining a practical length.

In Figures 6-26 and 6-27. the performance of MAP demodulation is compared with that of soft decision decoding of differentially detected QPSK. A number of points are apparent:

- the simple soft decisions from a differential detector work quite well. outperforming the soft decisions from the MAP demodulator when the pilot symbol rate is $1: 16$ :

- the improved soft decisions from the MAP demodulator with a pilot symbol rate of $1: 8$ provide approximately $3 \mathrm{~dB}$ of gain at an error rate of $10^{-5}$. 
It is clear from the results that the error runs caused by phase slips are having a large impact on the ability of the convolutional decoder to correct the errors. Increasing the interleaver size would likely improve things: however, at the SNR of interest, the uncoded error rate is quite high so it may not be possible to sufficiendy increase the spacing berween errors at the input to the decoder.

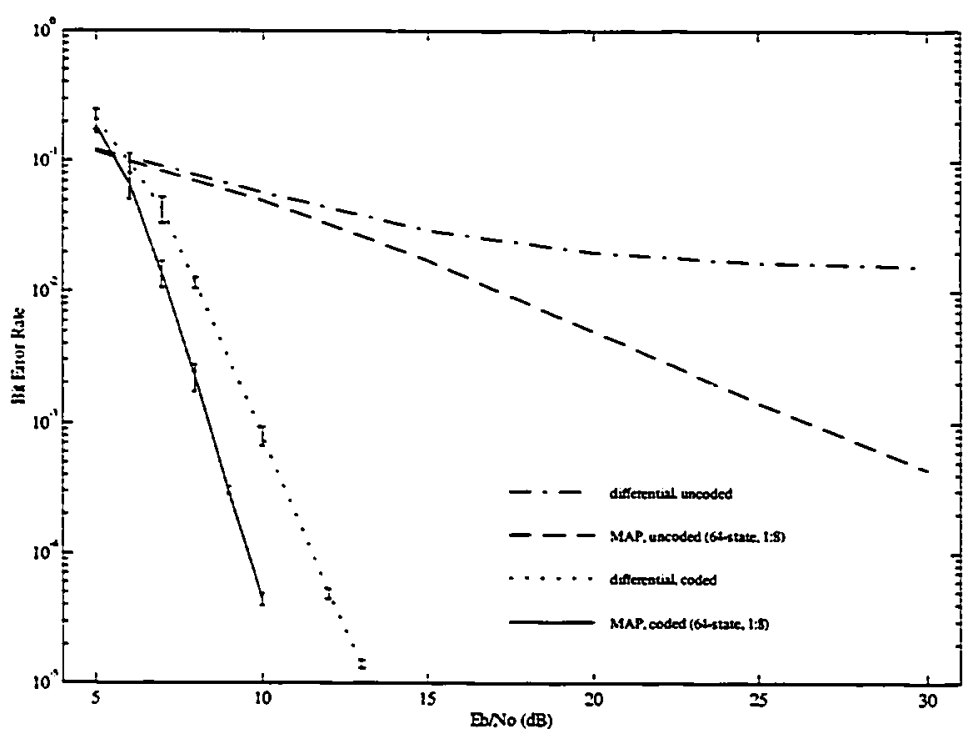

Figure 6-26 The coded and uncoded BER performance of the MAP demodulator for QPSK with a pilot symbol rate of $1: 8$ as compared to that of differential detection of differentially encoded QPSK. The fading rate is $0.05 R$. 


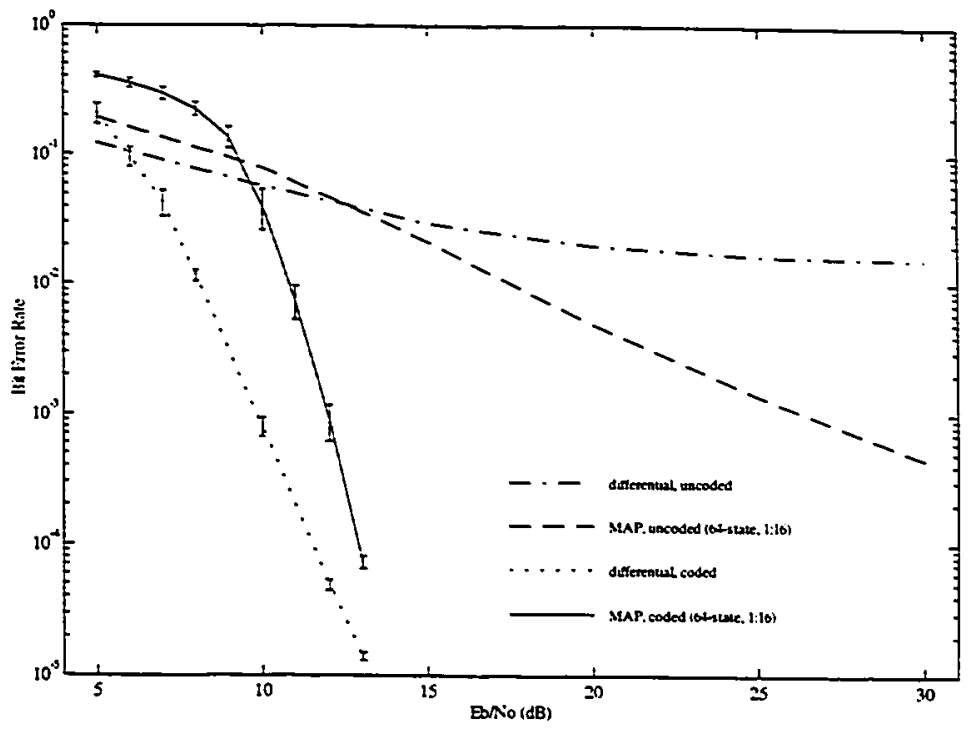

Figure 6-27 The coded and uncoded BER performance of the MAP demodulator for QPSK with a pilot symbol rate of 1:16 as compared to that of differential detection of differentially encoded QPSK. The fading rate is $0.05 R$.

While rectangular pulse shaped QPSK was used for the above simulations, the results are generally applicable to any scenario where the fading does not decorrelate the pulse after matched filtering at the receiver, i.e., where a sample rate of 1 sample per symbol is valid. From [36], a fading rate of $0.05 R$ requires rolloffs of $50 \%$ and above for this assumption to be valid. This requirement is consistent with the modulations employed in many mobile communications systems.

\subsection{Summary Of Demodulation Results}

The symbo!-by-symbol MAP demodulator exhibits good performance over the range of channel conditions studied. In an uncoded system, no irreducible error rate is observed even for an $E_{b} / N_{0}$ of $30 \mathrm{~dB}$ at a fading rate of $0.3 R$ and the BER estimates produced by 
the demodulator closely match the measured BER. While pilot symbols are required for use with QPSK, their rate is determined only by phase ambiguity considerarions rather than being directly tied to Nyquist sampling the fading process.

The performance resuits for the symbol-by-symbol MAP demodulator feeding a convolutional decoder show the demodulator's soft decisions to produce a $3-4 \mathrm{~dB}$ performance improvement over differential detection at the lower fading rate $(0.05 R)$ and to remain robust at the higher fading rate $(0.3 R)$ when the differential detector is unusable.

The symbol-by-symbol MAP demodulator is amenable to reduced state implementation. largely insensitive to errors in the modelled additive noise level, and moderately sensitive to errors in the modelled fading bandwidth. Hard decision performance is unaffected by mismatches between the actual and expected squared prediction error: however, soft decisions, and therefore BER estimation, are biased. The use of decision direction in estimating the squared prediction error should eliminate this bias if it is problematic. 


\section{Chapter 7}

\section{Iterative MAP Processing For Joint Demodulation And Decoding}

In this section we examine the possibility of using iterative demodulation and decoding to improve the performance of the symbol-by-symbol MAP demodulator. Because it utilizes a priori probabilities and because the soft decisions produced by the demodulator are inherently symbol (or bit) probabilities, the MAP demodulator is well-suited to iterative processing applications where the demodulator is explicitly niciuded in the iterative processing.

\subsection{Goal Of The Iterative Processing}

The major impairments facing the symbol-by-symbol MAP demodulator are:

a) the additive white gaussian noise level as determined by the $E_{b} / N_{0}$ :

b) the amplitude variations inuroduced by the fading process: and,

c) the phase variations introduced by the fading process.

In the simulated MAP receiver, the effect of the first two impairments is combatted with coding and interleaving while the effect of the phase variation is handled via the linear predictor. If the predictor were perfect. the demodulation problem would in essence reduce to the detection of a signal with a Rayleigh faded amplitude component in AWGN. In fact. Lodge and Moher show in [33] that for a bandlimited fading process, the variance of the prediction error can be made arbitrarily small by choosing a predictor of sufficient order. In reality of course, an arbitrarily large predictor order is not an option; however, we know from the simulation results of Section 6.2.5 that the predictor order (as 
manifested in the variance of its channel estimates) does have a definite impact on the performance of the demodulator.

Consider the filter bank structure of Figure 5-4. The prediction errors for each hypothesis form the input to the MAP processing. The probabilistic nature of these errors means that for certain symbol periods, the estimated prediction error from one or more incorrect hypotheses can be less than the prediction error estimated from the correct hypothesis. The frequency with which this error scenario occurs, and the expected magnitudes of the errors. are proportional to the variance of the prediction error. In the absence of other information, the MAP processing assumes that all hypotheses have equal a prior probabilities: therefore, the nature of the crrors described above essentially determine the performance of the demodulator.

The variance of the output of the prediction error filters is determined by both the accuracy of the predictor. and the variance inherent in the random process being modelled. While variance due to the former can be reduced by increasing the precictor order. the same does not hold for the variance due to the latter since it is largely caused by AWGN.

An alternative to increasing the accuracy of the prediction is to increase the information provided to the MAP processing by weighting the prediction errors input to the algorithm by more accurate a priori probabilities for each hypothesis. A corresponding improvement in demodulator performance would be expected. The goal of the iterative processing is therefore to provide the demodulator with more accurate a priori symbol probabilities so that its soft decisions are based on more accurate channel estimation.

\subsection{General Concept}

As described in Section 1.1.1, the goal of multistage soft-in soft-out processing is that the performance of the overall receiver will approach that of optimal single stage joint demodulation and decoding. The major problem with this multistage approach is that while later stages can use the information gleaned from previous stages, the reverse is not generally true. This motivates an iterative processing approach which allows earlier stages to refine their processing based on information from later stages. 
The operation of an iterative processing system can be thought of in terms of the information that each stage adds to the final result. As an example. consider the operation of a demodulator. The goal of the demodulator is to produce soft decisions which reflect the probability that a given bit is a 0 or a 1 . The information available to the demodulator is the received signal. $y(\tau)$. and the initial a priori probabilities of the input bits (which are generally 1/2). The demodulator uses this information. combined with knowledge of the chosen modulation and of the channel structure. to produce its soft decisions.

Viewed mathematically on a bit-by-bit basis. the demodulator wishes to determine the a posteriori bit probabilities

$$
\operatorname{Pr}\left(u_{n}=0 \mid y(\tau)\right)=\frac{p\left(y(\tau) \mid u_{n}=0\right) \cdot \operatorname{Pr}\left(u_{n}=0\right)}{p(y(\tau))}
$$

and

$$
\operatorname{Pr}\left(u_{n}=11 y(\tau)\right)=\frac{p\left(y(\tau) \mid u_{n}=1\right) \cdot \operatorname{Pr}\left(u_{n}=1\right)}{p(y(\tau))}
$$

for all $\left\{u_{n}\right\}$ (this is essentially the same as (5.1) in the derivation of the symbol-by-symbol MAP demodulator). Equation (7.2) embodies the bit soft decision as represented thus far in this thesis. Two other equally valid representations are the likelihood ratio and the log likelihood ratio. Consider the likelihood ratio formed from the a posteriori probabilities of (7.1) and (7.2).

$$
\begin{aligned}
L_{n} & =\frac{\operatorname{Pr}\left(u_{n}=1 \mid y(\tau)\right)}{\operatorname{Pr}\left(u_{n}=0 \mid y(\tau)\right)} \\
& =\frac{p\left(y(\tau) \mid u_{n}=\mathbf{1}\right)}{p\left(y(\tau) \mid u_{n}=0\right)} \cdot \frac{\operatorname{Pr}\left(u_{n}=1\right)}{\operatorname{Pr}\left(u_{n}=0\right)}
\end{aligned}
$$

We can see that this ratio is in fact the product of two distinct quantities. The first term. being conditioned on a particular value of $u_{n}$ is independent of the a priori probabilities for the bit at time $n$, although it may be dependent upon the a priori probabilities for bits transmitted at times other than time $n$. The second term embodies the $a$ priori

probabilities for $u_{n}$. 
For clarity of exposition, it is useful to rewrite (7.3) using logarithms of the likelihood ratios. Converting to a vector notation to eliminate the $n$ subscript. we get

$$
\mathbf{R}^{\boldsymbol{c}}=\mathbf{R}^{i}+\mathbf{R}^{\mathbf{a}} \text {. }
$$

where $\mathbf{R}^{c}$ is the composite $\log$ likelihood ratio. $\mathbf{R}^{i}$ is the $\log$ likelihood ratio of the socalled intrinsic information (a term introduced in [37] to refer to the information that the demodulator gleans about $u_{n}$ from the input signal and potentially the a priori probabilities of the other transmitted bits, without utilizing the a priori probability for $u_{n}$ ) and $\mathbf{R}^{a}$ is the $\log$ likelihood ratio of the a priori information.

As an example, consider the receiver portion of Figure 7-1 where the demodulator is stage 0 , the first decoder is stage 1 and the second decoder is stage 2 . The inputs to the first decoder are the soft decisions embodying the a priori bit probabilities, and the intrinsic information obtained by the demodulator. The decoder utilizes its knowledge of the code structure to produce a refined set of soft decisions (input bit probabilities). From an information perspective, the output of this stage can be represented as

$$
\mathbf{R}^{c}(1)=\mathbf{R}^{i}+\mathbf{R}^{c}+\mathbf{R}^{e}(1)
$$

where $R^{c}(1)$ is the composite log likelihood ratio after the processing of stage 1 (the first decoder), and $R^{e}(1)$ is the extrinsic information added by stage 1 (information added by a stage other than the demodulator which is independent of the input soft decision at time

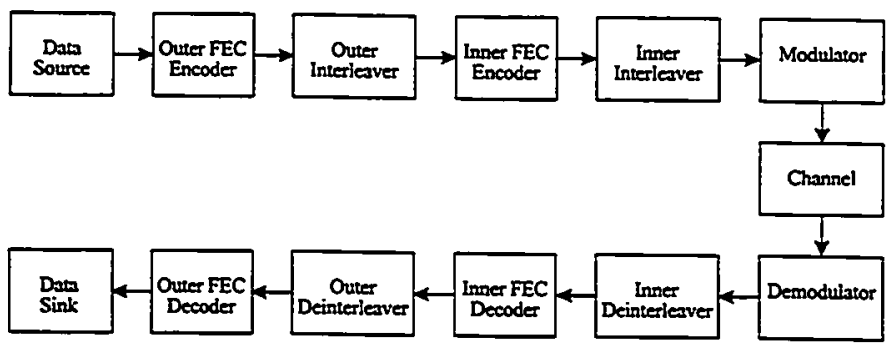

Figure 7-1 An example of a system utilizing concatenated processing techniques. 
$n$ but utilizes the soft decisions for other bits). When $R^{c}(1)$ is passed as the input to stage 2 (the second decoder), the sum $\mathbf{R}^{a}+\mathbf{R}^{r}(1)$ can be viewed as augmented a priori information to be urilized by stage 2 .

It is important to note that (7.5) only holds if the inputs to the decocer are independent (if the inputs are not independent, then the log likelihood ratios may not be factored into separate terms). If the channel has memory. this independence assumption will not be valid: therefore. interleaving must be present berween the demodulator and the decoder. The same argument holds for subsequent decoder stages. Since the extrinsic information produced by a decoder for the bit at time $n$ utilized the data bit a priori probabilities and the intrinsic information from the demodulator for all times $i \neq n$, the extrinsic information for the bits at any two times, $n_{1}$ and $n_{2}$. may be correlated. Fortunately, this correlation decreases as $\left|m_{1}-n_{2}\right|$ increases: therefore. interleaving can be utilized to spread correlated information outside of the memory of the subsequent decoder stage (in essence. a diversity effect is achieved since the exrinsic information at time $n$ is only weakly correlated to the a priori bit probability and the intrinsic information at time $n$ ).

The above concepts can be extended for any $D$ stage system utilizing soft-in soft-out processing, i.e.. the composite $\log$ likelihood ratio out of stage $d$ can be written

$$
\mathbf{R}^{c}(d)=\mathbf{R}^{i}+\mathbf{R}^{e}+\sum_{j=1}^{d} \mathbf{R}^{e}(j)
$$

for $1 \leq d \leq D$.

Consider now an iterative processing approach which includes the demoduiator. In iterative processing, the information input to a stage on the second and subsequent iterations must not inciude the information added by that stage in the previous iteration. In the case of the demodulator, the a priori information utilized therefore includes the initial a priori information and the extrinsic information added by the subsequent stages on the previous iteration, but does not include the intrinsic information previously determined by the demodulator. For the $p$ th iteration, the augmented $a$ priori information input to the demodulator can therefore be written 


$$
\mathbf{R}_{p}^{\alpha}(0)=\mathbf{R}^{\alpha}+\sum_{j=1}^{D} \mathbf{R}_{p-1}^{c}(j)
$$

while the corresponding composite log likelihood ratio out of the demodulator becomes

$$
\mathbf{R}_{p}^{c}(0)=\mathbf{R}_{p}^{i}+\mathbf{R}^{c} \div \sum_{j=1}^{D} \mathbf{R}_{m_{i}}(j)
$$

where $\mathbf{R}_{p}^{\prime}$ is the augmented intrinsic information provided by the demodulator on the $p$ th iteration. Similarly, the augmented a priori information input to stage $d$ on the pth iteration can be written

$$
\mathbf{R}_{p}^{u}(d)=\mathbf{R}^{e}+\sum_{j=1}^{d-1} \mathbf{R}_{p}^{e}(j)+\sum_{j=d-1}^{D} \mathbf{R}_{p-1}^{e}(j)
$$

while the corresponding composite log likelihood ratio out of stage $d$ is

$$
\mathbf{R}_{p}^{c}(d)=\mathbf{R}_{p}^{i}+\mathbf{R}^{\alpha}+\sum_{j=1}^{d} \mathbf{R}_{p}^{e}(j)+\sum_{j=d=1}^{D} \mathbf{R}_{p-1}^{e}(j)
$$

\subsection{Structure Of Iterative MAP Receiver}

The iterative MAP simulations utilize the same modulation. channel models and overall processing as the non-iterative simulations described in Chapter 6. As a result, the only portion of the simulation program which changes is the structure of the MAP receiver.

A block diagram of the iterative MAP receiver is shown in Figure 7-2. Processing is performed on interleaver blocks which may span one or more frames (one frame being the block size for the symbol-by-symbol MAP demodulator). When a block is received. it is passed through the bank of filters as in the conventional MAP receiver (see

Figure 5-4). The resulting $\left\{\gamma_{;}(k)\right\}$ are saved for later use. and then passed, with the a priori symbol probabilities. to the symbol-by-symbol MAP processing which determines the corresponding a posteriori probabilities. Note that the initial a priori symbol probabilities utilized by the demodulator are $1 / Q$ for all of the $Q$-ary symbols in data symbol periods. 1 for the pilot symbols in the pilot symbol periods, and 0 for all other symbols in the pilot symbol periods. The symbol probabilities produced by the 


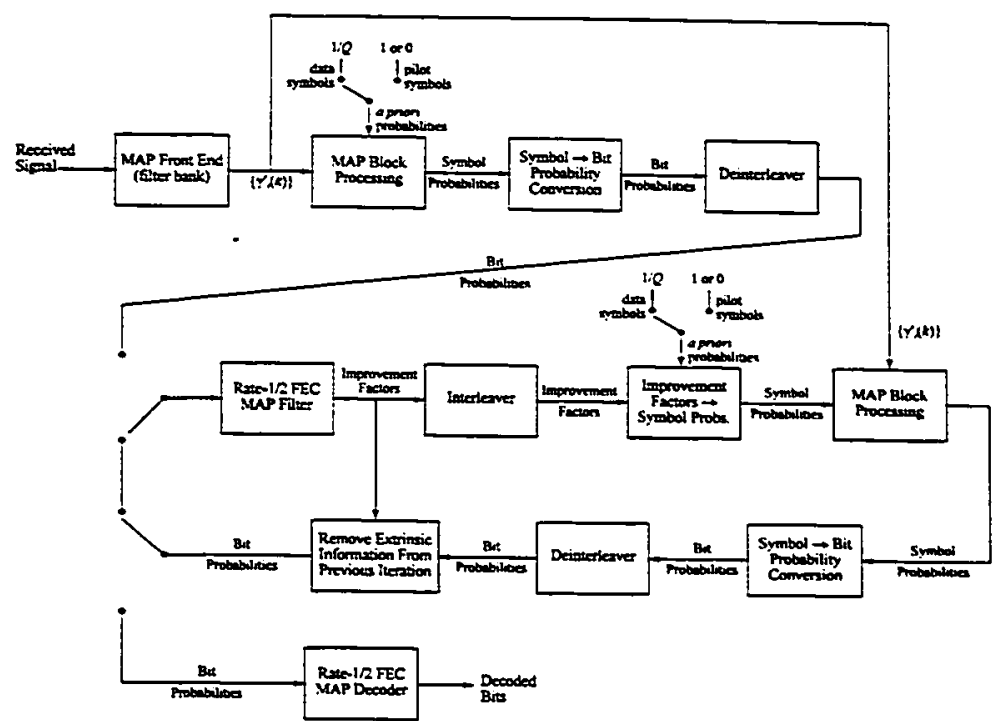

Figure 7-2 The structure of iterative MAP demodulation/decoding.

MAP processing are then converted to bit probabilities: these are the bit soft decisions produced by the demodulator. Note that the processing to this point is identical to that for the non-iterative receiver.

If only a single iteration is required (i.e.. the normal non-iterative processing case), the bit soft decisions are deinterleaved and then fed to a MAP convolurional decoder. If multiple iterations are required. the deinterleaved soft decisions are fed to a MAP filter. This filter utilizes its knowledge of the FEC coding to determine updated probabilities for all of the coded bits (c.f. a decoder which determines probabilities for all of the data bits). These updated probabilities are embodied in a set of improvement factors which relate the information at the output of the filter to the information at its input and thus represent the extrinsic information added by the filter. Using the ratio of the output and input likelihood ratios. we get 


$$
\begin{aligned}
& I_{f l t}(n)=\frac{L_{s, t}(n)}{L_{d m}(n)} \\
& =\frac{P_{0}(x(n)=01) / P_{g}(x(n)=0)}{P_{m}(x(n)-1) / P_{-}(x(n)=0)} \\
& =\frac{\mu_{f i t}(n) \cdot\left(1-\mu_{d m m}(n)\right)}{\left(1-\mu_{f l t}(n)\right) \cdot \mu_{d-m}(n)}
\end{aligned}
$$

where $\mu_{\mathrm{sm}}(n)=P_{\mathrm{dm}}(x(n)=1)$ is the probability at the input to the filter that the data bit at time $n$ is $\mathrm{I}$ and $\mu_{n a t}(n)=P_{f k t}(x(n)=1)$ is the comesponding probability at the output of the filter.

In preparation for the second pass. the improvement factors inust be related back to the input of the MAP block demodulator. This requires reinterleaving. and then using the improvement factors to produce augmented a priori probabilities. As desired. the improvement factors do not embody the intrinsic information generated by the demodulator on the first iteration: therefore. assuming equiprobable initial probabilities. the augmented a priori bit probabilities can be written

$$
\begin{aligned}
& P_{\text {aux }}(x(n)=\mathrm{I})=\frac{\mathrm{I}_{\text {fit }}(n)}{1+\mathrm{I}_{\text {fit }}(n)}
\end{aligned}
$$

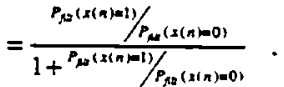

$$
\begin{aligned}
& =P_{\text {fti: }}(x(n)=1)
\end{aligned}
$$

The MAP block demodulator uses these new probabilities. and the branch updates from the filter bank. to compute new soft decisions. Note that the filter bank operation does not need to be performed again if the data can be stored.

On this iteration (and all subsequent iterations). the soft decisions output from the MAP demodulator must be processed to remove the extrinsic information generated by the MAP filter on the previous iteration. This is accomplished by converting soft decisions out of the demodulator to a likelihood ratio and then computing an adjusted likelihood ratio from 


$$
L_{s_{m, p}}(n)=\frac{L_{d m, p}^{\prime}(n)}{I_{n k, p-1}(n)}
$$

where $L_{d e m p}(n)$ is the likelihood ratio for the bit a time $n$ which is to be input to the filter or decoder on the $p$ th iteration. $L_{d e m, p}(n)$ is the corresponding likelihood ratio output from the demodulator on the $p$ th iteration. and $\mathrm{I}_{\text {fils- }-1}(n)$ is the corresponding improvement factor from the MAP filter on the previous iteration. This likelihood ratio may be easily converted to a soft decision using

$$
\mu_{d m_{m}, p}(n)=\frac{L_{d m_{m}, p}(n)}{1+L_{d m_{m}, p}(n)} \text {. }
$$

At this point the soft decisions may either be routed to the MAP filter for another iteration, or to the MAP decoder to generate hard decisions.

It should be noted that care must be taken when performing manipulations of probabilities and likelihood ratios: otherwise, information may be lost due to a loss of precision. or singularities (i.e.. $0 / 0$ ) may occur. In the simulations deseribed below. the latter problem is avoided by bounding probabilities to ensure that they never reach precisely 1 or 0 .

\subsection{Soft Decision Reference Receiver}

It is of interest to compare the BER performance of the MAP receiver with that of a fictitious receiver which produces perfect soft decisions. i.e.. soft decisions which have no phase ambiguity and whose magnitude is proportional to the square of the amplitude change introduced by the channel. No differential encoding or pilot symbols are required.

The equivalent channel model seen by the decoder in the ideal channel state receiver is shown in Figure 7-3. The received signal is multiplied by the complex conjugate of the multiplicative fading introduced by the channel giving 


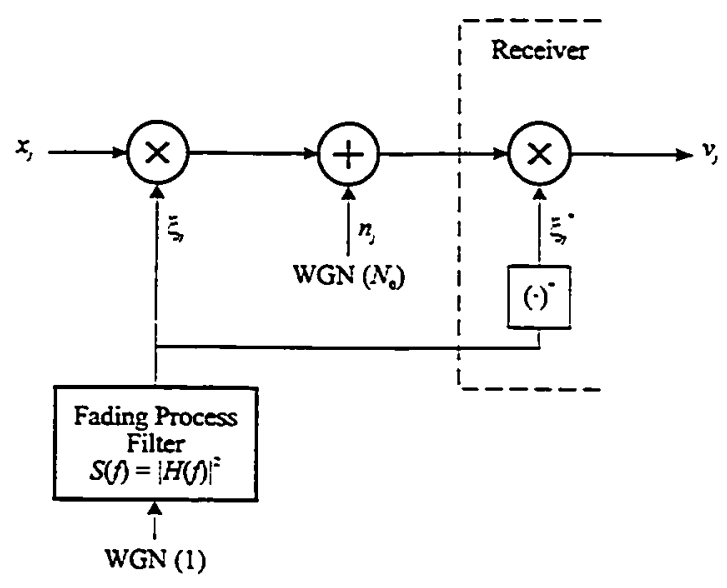

Figure 7-3 The equivalent channel model for the ideal channel state receiver.

$$
\begin{aligned}
v_{1} & =\left(x, \xi_{,} \div n_{3}\right) \cdot \dot{\xi}_{,} \\
& =x,\left.\xi_{,}\right|^{2} \div n, \dot{\xi}_{j}
\end{aligned}
$$

The resulting phasor. $y_{j}$, is fed into a Viterbi decoder which determines the maximum likelihood sequence. Note that $E\left[n, \bar{\zeta}_{j}^{-}\right]$is zero.

\subsection{Performance With QPSK Signals}

Simulations were performed to determine the performance of the iterative MAP receiver with QPSK signals. The simulation parameters were the same as for the non-iterative simulations. i.e.:

Fading rate: $0.05 R$

Sample rate: $r=1$ sample/symbol

Symbol encoding: Gray coding

QPSK pulse shaping: none ( $K=1$ symbol period)

MAP predictor order: 3 ird order $(\alpha=3$ symbol periods)

MAP pilot symbol rate: $1: \$, 1: 16$ 


$\begin{array}{ll}\text { Code rate: } & 1 / 2 \\ \text { Constraint length: } & 7 \\ \text { Interieaver. } & 12 \$ \times 32\end{array}$

The BER performance for the $1: 8$ pilot symbol rate is shown in Figure 7-4. Note that "I iteration" refers to the normal non-iterative approach: i.e., the results presented in Section 0 . Also shown on the figure is the performance of the fictitious reference receiver urilizing ideal channel state information.

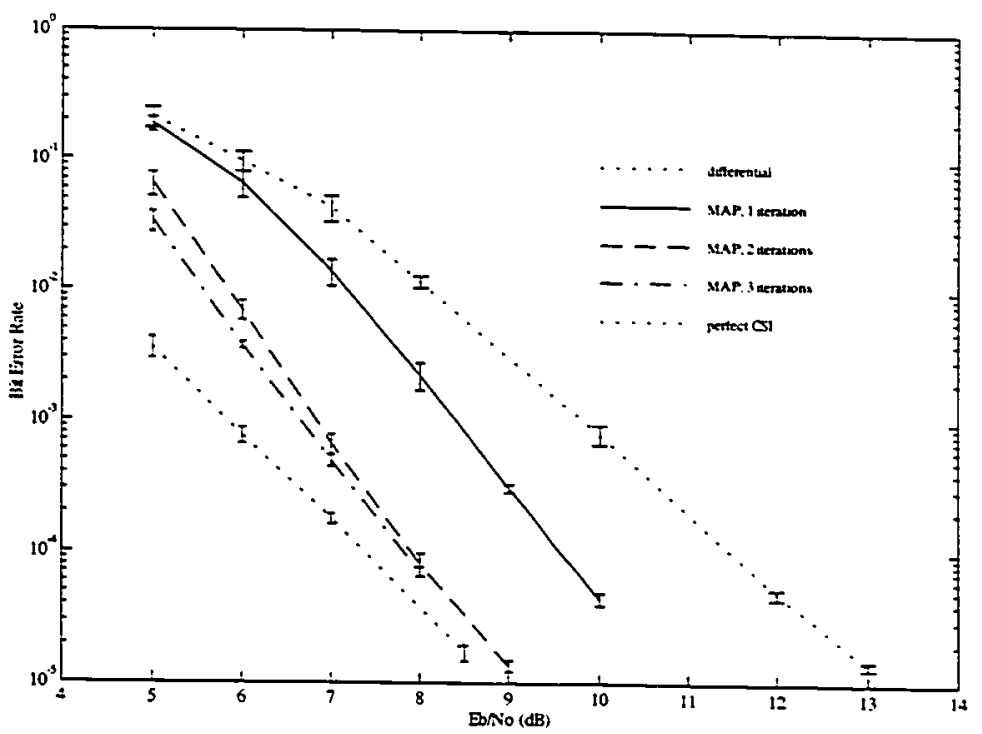

Figure 7-4 The BER performance of iterative MAP demodulation/decoding as compared to that of conventional differential detection and convolutional decoding for the reception of QPSK signals. Also stown is the performance of a fictitious reference receiver utilizing ideal channei state information. The fading rate is $0.05 R$ and the pilot symbol rate used in the MAP simulations is 1:8. 
A number of points can be noted:

- most of the improvement (1-1.5 dB in the range of interest) is obtained with a single additional iteration:

- while iterating provides some performance improvement at low $E_{b} / N_{0}$, the additive noise clearly inhibits the ability of the iterative processing to converge to accurate channel estimates (this can be seen by noting the significantly better performance of the ideal channel state receiver at these $E_{b} / N_{0}$ ):

- at an error rate of $10^{-5}$, the performance of the MAP receiver utilizing 2 iterations approaches that of decoding with ideal channel state information.

This last result is paricularly impressive. When the $0.5 \mathrm{~dB}$ overhead due to power in the pilot symbols is taken into account, the performance of the iterative MAP receiver matches, within error bars, that of the receiver utilizing ideal channel state information.

Since the performance difference between the iterative MAP receiver and the reference receiver is essentially wholly due to the power in the pilot symbols, it is desirable to reduce the pilot symbol rate and therefore the corresponding power penalty. To this end, the system utilizing the 1:16 pilot symbol rate was investigated. Recall that for this pilot symbol rate, the single iteration performance was worse than that obtained using simple differential detection. The resulting BER performance is shown in Figure 7-5. A number of points can be noted:

- most of the improvement ( $45 \mathrm{~dB}$ in the range of interest) is obtained with two or three additional iterations:

- at an error rate of $10^{-5}$. the performance of the MAP receiver utilizing 4 iterations approaches that of decoding with ideal channel state information.

Once again. this last result is particularly impressive. When the $0.26 \mathrm{~dB}$ of power in the pilot symbols is taken into account. the performance of the iterative MAP receiver matches, within error bars. that of the receiver utilizing ideal channel state information. 


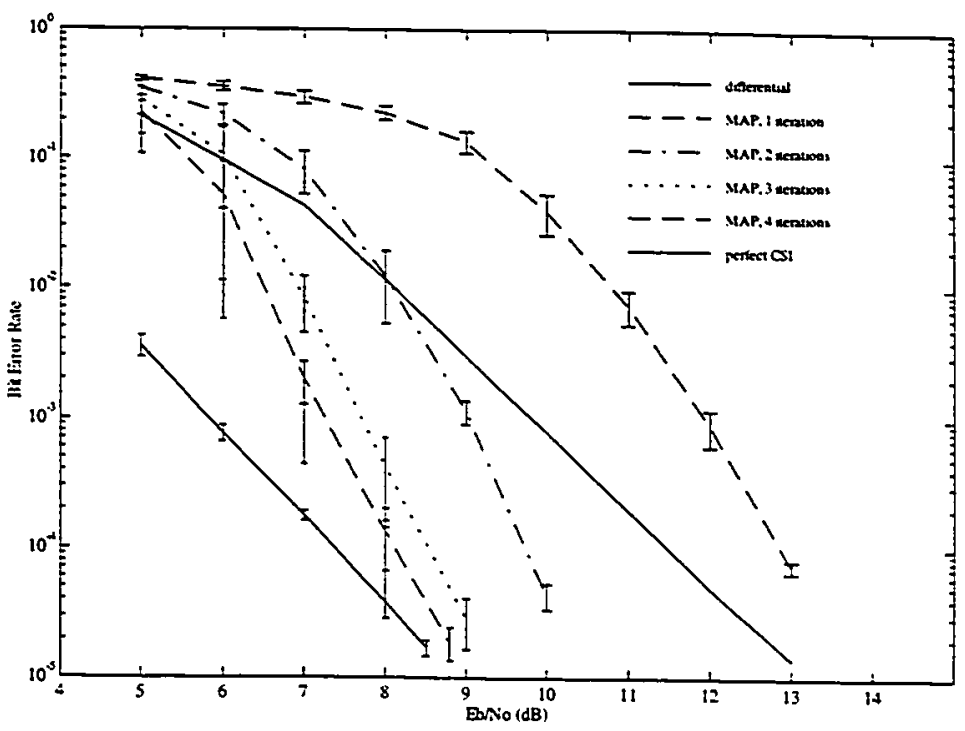

Figure 7-5 The BER performance of iterative MAP demodulation/decoding as compared to that of conventional differential detection and convolutional decoding for the reception of QPSK signals. Also shown is the performance of a fictitious reference receiver utilizing ideal channel state information. The fading rate is $0.05 R$ and the pilot symbol rate used in the MAP simulations is $1: 16$.

The difference in the results for the $1: 8$ and $1: 16$ pilot symbol rates shows that the iterative processing is particularly effective in combarting the effect of phase slips; this was a result which was anticipated. To see why, one can look at the performance of a convolutional decoder in a non-iterative system. When a decoder is operating above its threshold $E_{b} / N_{0}$. its decisions tend to have occasional error bursts interspersed with largely correct data: this property is amplified as the $E_{b} / N_{0}$ increases. Since a MAP filter is essentially a decoder without its final decoding stage, its output exhibits the same characteristic except that the error bursts are bursts of bits whose confidence level is low (bits with $\operatorname{Pr}(x=1) \approx 05$ ). When the output of the MAP filter is reinterleaved, both the low confidence and high confidence probabilities are distributed across the interleaver 
block. Bits with probabilities near 0.5 will generally be associated with bits which had similar probabilities out of the demodulator or oceurred during phase slips (and therefore failed to line up with the FEC code). More importantly, the majority of the bits (which should have probabilities near 1) serve as almost-known symbols and aid in resolving phase ambiguities in the same way that pilot symbols do.

It should be noted that the performance of iterative processing is quite dependent upon the depth of interleaver since the reinterleaved probabilities represent a form of time diversity at the front end of the demodulator. If too small an interleaver is used, an insufficient number of high confidence probabilities will be interspersed into bursts of poorer probabilities.

One more obvious characteristic of the results shown in Figure 7-5 is the large error bars associated with some of the iterative processing results. While the error bars used in this thesis are intended to signify a $98 \%$ confidence interval, this meaning only holds if the error events have an approximately Gaussian distribution (see Appendix B - Simulation BER Confidence Intervals). For the higher BER's at the 1:16 pilot symbol rate, what was in fact observed was that the results were dominated by errors in a few interleaver blocks which had a particularly large number of bit errors. The consequences of this are not only that the BER's are perhaps worse than some sort of corresponding block error rate, but also that the run-to-run statistical variation is larger, resulting in the error bars shown.

This behaviour is to be expected in an iterative processing system. If a large number of errors are present, the decoder may start to converge towards an incorrect sequence. In this case. the interleaving can have a negative effect and actually spread bad information through much of the block. The problem is exacerbated when the probabilities for the incorrect data start to approach 1 since the algorithm tends to lock on to bits with high confidence levels.

\subsection{Summary}

The use of iterative processing techniques for joint demodulation and decoding can produce improvements of up to $5 \mathrm{~dB}$ over the QPSK BER performance of the noniterative MAP receiver. The number of iterations required is a function of both the error 
rate and its distribution. With a sufficient number of iterations, the performance of the iterative MAP receiver can approach that of a receiver utilizing ideal channel state information. 


\section{Chapter 8}

\section{Conclusion}

\subsection{Summary}

The problem of producing good soft decisions for CPM signals transmitted over Rayleigh flat-fading channels was investigated. The work was motivated by the increasing importance of soft-output demodulators in systems utilizing concatenated processing.

The symbol-by-symbol MAP demodulator models the system as a trellis whose state represents the bits affecting the received signal at any given time. The trellis branch weights represent the product of the a priori symbol probability and the probability density of the present received signal sample. conditioned upon the past received signal samples and the subset of hypothesized input symbols that form the state vector. The demodulation problem then becomes one of efficiently computing, via the MAP algorithm. the state transition probabilities for all times $t$, and then obtaining symbol soft decisions by summing those probabilities that correspond to branches associated with a particular symbol $q$ at time $t$.

The performance of the symbol-by-symbol MAP demodulator was evaluated via simulation for both binary CPM and QPSK signals. Its application in an iterative demodulation/decoding receiver was also introduced and then evaluated by simulation. 


\subsection{Results}

The simulations performed lead to the following conclusions:

1. The symbol-by-symbol MAP demodulator exhibits good performance over the range of channel conditions studied.

2. With binary CPM at a fading rate of $0.3 R$. differential detection has become essentially useless while the MAP demodulator shows no sign of an irreducible error floor, even at bit error rates of $10^{-4}$.

3. When the PEF is matched to the channel. the BER estimate produced by the MAP algorithm closely matches the BER measured in the simulation.

4. With rate-1/2 coding at a fading rate of $0.05 R$. differential detection of binary CPM produces relatively good soft decisions (given the simplicity of the approach); however, the symbol-by-symbol MAP demodulator still provides an additional $4 \mathrm{~dB}$ of gain at an error rate of $10^{-5}$.

5. With rate-1/2 coding at a fading rate of $0.3 R$. differential detection of binary CPM is completely ineffective while the symbol-by-symbol MAP demodulator continues to provide good performance.

6. Average mutual information provides a means of analyzing the quality of soft decisions produced by various demodulators. An analysis of the hard and soft decisions produced by the differential detector and MAP demodulator demonstrates the superiority of the MAP approach.

7. Reduced state implementations of the MAP algorithm are desirable due to its inherent complexity. The number of states required can be reduced by truncating the CPM pulse shape. or by adjusting the order of the channel PEF.

8. While, as expected, configurations utilizing a larger number of states provide better performance, the difference is small at the lower $E_{b} / N_{0}$. Furthermore, so long as the PEF remains matched to the channel, reduced state symbol-by-symbol MAP processing still produces accurate probability estimates. 
9. The uncoded BER performance of the symbol-by-symbol MAP demodulator is largely insensitive to errors in the modelled additive noise level, and moderately sensitive to errors in the modelled fading bandwidth.

10. The BER estimation and soft decision performance of the symbol-by-symbol MAP demodulator is sensitive to errors in the expected prediction error. There are a number of ways of computing this error to avoid problems.

11. At one sample per symbol, QPSK can be considered a discrete-time form of CPM. While pilot symbols are required for use with QPSK, their rate is determined only by phase ambiguity considerations rather than being directly tied to Nyquist sampling the fading process.

12. As in the binary CPM case, differential detection of QPSK results in an irreducible error floor which is not evident (at bit error rates of $5 \times 10^{-5}$ ) in the MAP demodulator results.

13. When the pilot symbol rate is insufficient to avoid phase slips, the rate-1/2 coded BER performance of the differential detector is better than that of the MAP demodulator, however, at the $1: \$$ pilot symbol rate, the MAP demodulator outperforms differential detection by approximately $3 \mathrm{~dB}$ at an error rate of $10^{-5}$.

14. Because it can utilize a priori information, and because the soft decisions produced by the demodulator are inherently symbol (or bit) probabilities, the MAP demodulator is well-suited to iterative processing applications.

15. With rate-1/2 coded QPSK and a 1:8 pilot symbol rate. a 1-1.5 dB improvement is obtained with a single additional iteration.

16. With rate-1/2 coded QPSK and a $1: 16$ pilot symbol rate, a $5 \mathrm{~dB}$ improvement is obtained with 3 additional iterations. illustrating the ability of iterative processing to combat the effect of phase slips.

17. At both the $1: S$ and 1:16 pilot symbol rates. the resulting performance is within a small fraction of a dB (the additional power in the pilot symbols) of that of a receiver utilizing ideal channel state information. 


\subsection{Summary Of Contributions}

This thesis encompasses the following core contributions:

1. The symbol-by-symbol MAP algorithm has been derived for the demodulation of CPM signals on Rayleigh flat-fading channels.

2. An efficient solution was derived to the generic computational problem of computing. for each trellis branch. the sum of the products of the weights of all paths through the trellis which pass through that branch.

3. The information content of soft decisions from a differential detector has been compared to that achievable using the symbol-by-symbol MAP demodulator. clearly demonstrating the superiority of the MAP approach at high fading rates.

4. A receiver structure utilizing iterative demodulation and decocing was derived and implemented in simulation.

5. Simulations were run which demonstrated that, at bit error rates of interest for mobile communications, the performance of iterative MAP demodulation/decoding can approach that of a demodulator utilizing ideal channel state information.

\subsection{Suggestions For Future Research}

The results of this thesis pose a number of interesting questions for future research:

1. Investigation of reduced-state implementations

The symbol-by-symbol MAP demodulator has shown itself to be amenable to reduced state implementation. Further investigation is required to characterize the performance tradeoffs associated with the various approaches, particularly in iterative processing applications.

The iterative processing receiver also affords the possibility of utilizing different numbers of states for different iterations.

2. Characterization of performance with a non-ideal receiver

The performance of the symbol-by-symbol MAP demodulator should be evaluated in the presence of frequency, timing and channel estimation error. For the latter. the 
performance with an adaptive channel estimator is of particular interest. A byproduct of these results would be an indication of whether a slightly more complex computation of the expected prediction error is warranted (i.e., one which is more decision-directed rather than relying on the correctness of the model). This is important since the soft decisions output by the symbol-by-symbol MAP demodulator are biased by any errors in the expected prediction ertor.

3. Investigation of sub-optimal soft-decision algorithms

While the effectiveness of both the standard symbol-by-symbol MAP demodulator. and the iterative demodulation/decoding receiver in reducing BER under fast fading conditions is evident. the performance improvements come at the cost of increased receiver complexily. The use of other algorithms to solve the branch probability problem should be investigated. The work in [38]. [39]. [40] and [41] provides starting points for this research while the results presented in this thesis serve as a good baseline.

4. Application to other modulations and channels

The techniques reported in this thesis follow from those of [33] which assumes constant modulus signalling and flat-fading. It is anticipated that these techniques can be generalized to non-constant modulus signalling and frequency selective fading. in ways similar to the generalizations of [33] published in [42] and [43]. Similar applicability of the techniques to implementations utilizing antenna diversity would also be expected.

5. Investigation of MAP processing in time and frequency

The use of symbol-by-symbol MAP processing in multidimensional demodulation (e.g.. demodulation of multi-carrier modulations in time and frequency) could be investigated. Here, it is expected that complexity considerations may quickly dominate and reduced state processing may be required. Altematively, iterative approaches using multiple passes in time and frequency may approach the performance of the combined solution with significantly less computational complexity. 


\section{Appendix A}

\section{CPM Matched Filter Requirements}

This appendix expands on the results in [33] which determine the form of the matched filters used in the computation of the $B_{j}(k)$ 's. The filters perform two tasks:

- removal of the modulation for hypothesis $k$ :

- determination of the channel prediction error using a $L$ th order linear predictor.

From (5.21) we have

$$
B_{j}(k)=\left|\sum_{i=0}^{L} a_{l}^{L} x_{j-1}^{-}(k) y_{j-1}\right|^{2}
$$

where the summation term implements a length $L+1$ FIR filter which accomplishes both of the above tasks.

Assuming that the modulator pulse length is finite. only a finite number of information symbols can affect the samples utilized in the prediction error filter, i.e., samples $x_{,-L}(k)$ through $x_{j}(k)$. We wish to determine the appropriate number of symbols.

In the discussion below, let: 


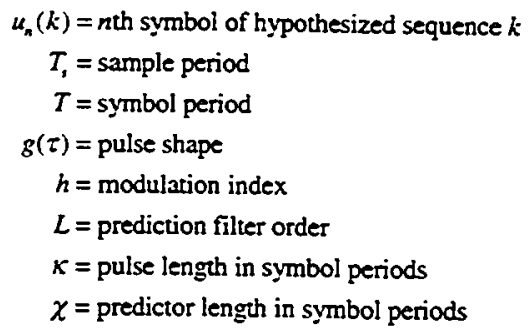

From (2.2) and (2.3). and assuming unity power per symbol, the complex baseband samples of the CPM signals of interest can be represented $3 s^{1}$

$$
x_{j}(k)=\exp \left[-j 2 \pi d \tau \int_{0}^{j \pi_{0}} \sum_{n} u_{n}(k) g(\tau-n T) d \tau\right]
$$

where

$g(\tau)=0$. for $\tau<0$ and for $\tau \geq \kappa T$.

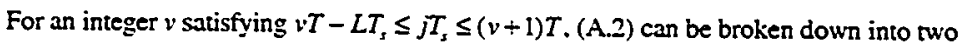
terms

$$
x_{j}(k)=x_{j}^{p}(k) \tilde{z}_{j}(k)
$$

where

$$
\begin{aligned}
& x_{j}^{p}(k)=\exp \left[-j 2 \pi h \int_{0}^{v T-L T_{s}} \sum_{n=0}^{s_{1}} u_{n}(k) g(\tau-n T) d \tau\right] . \\
& z(k)=\exp \left[-j 2 \pi h \int_{v T-L T_{s}}^{\pi s} \sum_{n=s_{n}}^{s_{1}} u_{n}(k) g(\tau-n T) d \tau\right] . \\
& s_{1}=\left\lfloor\left(v T-L T_{s}\right) / T\right\rfloor . \\
& s_{2}=\left\lfloor\left(v T-L T_{s}-\kappa T\right) / T+1\right\rfloor
\end{aligned}
$$

and

'Note that $j=\sqrt{-1}$ is not the same as $j$. the time index. 


$$
s_{3}=\left\lfloor j T_{s} / T\right\rfloor .
$$

The limits on the above summations are a function of the finite length of the pulse shape. Their derivation appears in Appendix B of [33]. Substituting (A.3) into (A.1). we get

$$
\begin{aligned}
& B_{j}(k)=\left|\sum_{i=0}^{L} a_{j}^{L}\left(x_{j-1}^{p}(k) z_{j-1}(k)\right)^{*} y_{j-1}\right|^{2} \\
& =\left|\sum_{i=0}^{L} a_{j}^{L}\left(x_{j-i}^{p}(k)\right)^{*} \dot{j}_{j-i}^{*}(k) y_{j-i}\right|^{2} \\
& =\left|\sum_{i=0}^{L} a_{j}^{2}\left(x^{p}(k)\right)^{0} \dot{j}_{j-t}^{-1}(k) y_{j-t}\right|^{2}
\end{aligned}
$$

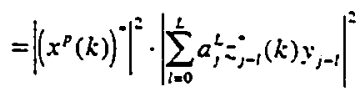

Since the $x_{j}^{p}(k)$ term has magnitude one and is independent of $j$ for

$v T-L T_{s} \leq j T_{s} \leq(v+1) T$, we can eliminate the first term of (A.6), leaving

$$
B_{j}(k)=\left|\sum_{l=0}^{L} a_{j}^{2} \bar{z}_{j-l}^{*}(k) y_{j-l}\right|^{2} .
$$

The computation of (A.7) for a given sample $j$ is affected by information from $s_{3}-s_{2}+1$ symbols. For the $\left\{j\right.$ comprising a given symbol interval. $v T \leq j T_{1}<(v+1) T$, the computation of (A.7) is affected by at most 


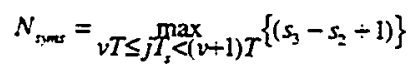

$$
\begin{aligned}
& ={ }_{v T \leq \max _{3}<(v+1) T}\left\{s_{3}\right\}-s_{2} \div 1 \\
& =\max _{v T \leq j T_{3}<(v \div 1) T}\left\{\left[\frac{j T_{t}}{T}\right]\right\}-\left[\frac{\left(v T-L T_{+}-\kappa T\right)}{T} \div 1\right] \div 1 \\
& =\left\{\begin{array}{cc}
\left\lfloor\frac{v T}{T}\right\rfloor-\left\lfloor v-\frac{L T}{T}-\kappa\right\rfloor & T_{s}=T \\
\left.\mid \frac{(v+1) T-T_{p}}{T}\right\rfloor-\left\lfloor v-\frac{L T_{s}}{T}-\kappa\right\rfloor & T_{s}<T
\end{array}\right. \\
& =v-\left\lfloor v-\frac{L T_{1}}{T}-\kappa\right) \\
& =-\left\lfloor-\frac{L T_{1}}{T}-\kappa\right\rfloor \\
& =\lceil\kappa+\chi\rceil
\end{aligned}
$$

symbols where $\chi=L T_{\mathrm{s}} / T$ is the predictor length in symbol periods. $\lceil a\rceil$ represents the smallest integer greater than or equal to $a$. and $\lfloor a\rfloor$ represents the largest integer less than or equal to $a$. This dependency is illustrated graphically in Figure A-1.

From (A.5). (A.6) and (A.8), we can see that the ealculation of $B_{j}(k)$ depends on the most recent $\lceil\kappa+\chi\rceil$ symbols of the kth hypothesis. If we assume that the most recent symbol is not embodied in a state, then the number of trellis states required becomes $Q^{[x+x\}-1}$.

To produce a time-invariant filter which computes (A.6). we must find a form of the required $\left\{z_{1}(k)\right\}$ which is time-invariant for the given hypothesis $k$. Since we only require the samples corresponding to the most recent $\lceil\kappa+\chi\rceil$ symbols of hypothesis $k$. any sequence of samples ending in the same $r \cdot\lceil\kappa \div \chi\rceil$ samples of $z,(k)$ can be used. 


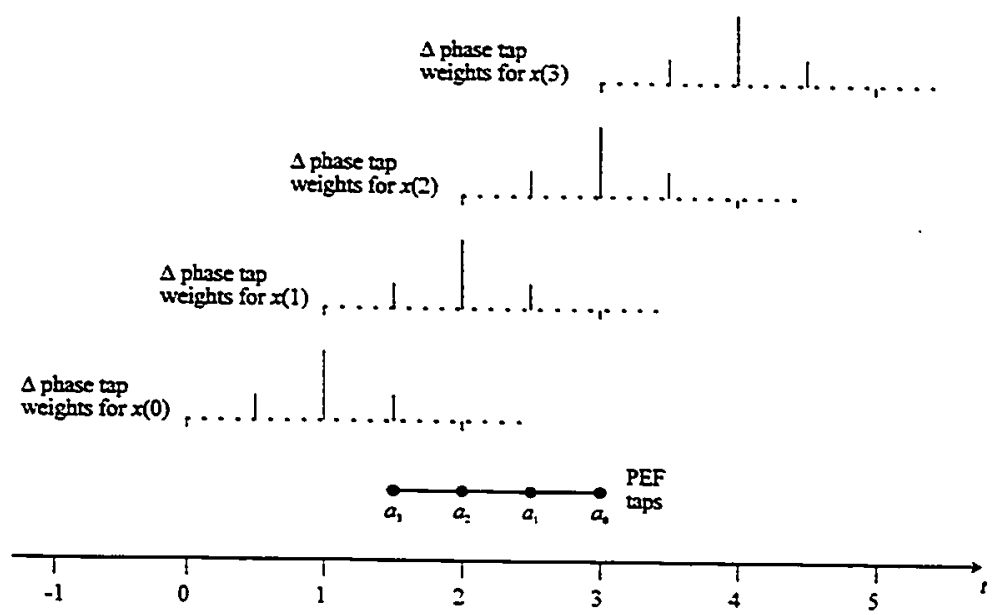

Figure A-1 A graphical representation of the symbols affecting the prediction error filter (PEF) calculation at $t=3$ for $K=2.5$ symbol periods. $\chi=1.5$ symbol periods and $r=2$ samples/symbol.

We can therefore write

$$
\sum_{i=0}^{r-1} B_{j-i}(k)=\sum_{i=0}^{r-1}\left|\sum_{i=0}^{L} h_{l}(s, i) y_{j-i-i=t}\right|^{2}
$$

where

$$
h_{l}(s . i)=a_{l}^{2} \dot{z}_{s-i-i=1}^{*}(s) .
$$

$s$ is any hypothesis for which the first $\lceil\kappa+\chi\rceil$ symbols are the same as the most recent

$\lceil\kappa+\chi\rceil$ symbols of hypothesis $k$, and $N=(\lceil\kappa+\chi\rceil-1) r$. 


\section{Appendix B}

\section{Simulation BER Confidence Intervals}

This appendix summarizes the statistics underlying the confidence intervals assigned to the results of simulation runs. It is based on a similar discussion in [22].

For each simulated scenario, the simulation program performs eight independent runs of at least 100 etror events per run. A bit error probability is measured for each run. At the conclusion of $N_{r}$ runs, the average probability of error is computed from

$$
\bar{P}_{e}=\frac{1}{N} \sum_{p=1}^{N} P_{e}(j)
$$

where $P_{e}(j)$ is the probability of error for run $j$.

Reference [44] describes how to obtain a confidence interval for the measured mean of a small set of anproximately nomally distributed samples with unknown variance. The confidence interval is given by

$$
\bar{x}-\frac{t_{\alpha / 2}}{\sqrt{n}} s<\mu<\bar{x}+\frac{t_{\alpha / 2}}{\sqrt{n}} s
$$

where $\bar{x}$ is the measured mean. $s$ is the standard deviation of the sample means, $n$ is the number of samples. $t_{\alpha s}$ is the value of the $t$ distribution with $v=n-1$ degrees of freedom leaving an area of $\alpha / 2$ to the right. and $\mu$ is the voue mean. In our application,

$$
\bar{x}=\bar{P}_{c}
$$

as shown above and 


$$
\begin{aligned}
s & =\sqrt{\frac{\sum_{j=1}^{N} P_{r}^{2}(j)-N_{r} \cdot \bar{x}^{2}}{N_{r}-1}} \\
& =\sqrt{\frac{\sum_{j=1}^{N} P_{r}^{2}(j)-\frac{1}{N_{r}} \cdot\left(\sum_{j=1}^{N} P_{e}(j)\right)^{2}}{N_{p}-1}} .
\end{aligned}
$$

The average probability of error is therefore given by

$$
\bar{x} \pm \frac{t_{a r}}{\sqrt{N}} s
$$

If $N=8$ and a $98 \%$ confidence interval is desired. then

$$
\frac{t_{\alpha I}}{\sqrt{N}}=\frac{t_{0.01 .7}}{\sqrt{8}}=1.06=1
$$

From (B.5) and (B.6), the true probability of etror lies in the range $\bar{x} \pm s$ with $98 \%$ confidence.

The derivation above is based on the assumption that the random variable, i.e. the measured mean. is normally distributed. To show this, we assume that the bit detection process in the simulated receiver is equivalent to a Bernoulli trial with one outcome being a bit error, and the other outcome being a correct bit.

If the probability of bit error is $p$, then the probability of $k$ errors in $n$ independent bits is $\left(\begin{array}{l}n \\ k\end{array}\right) p^{k}(1-p)^{n-k}$. If $n p(1-p) \gg 1$ then the DeMoivre-Laplace theorem states that

$$
\left(\begin{array}{l}
n \\
k
\end{array}\right) p^{k}(1-p)^{n-k} \approx \frac{1}{\sqrt{2 \pi n p(1-p)}} e^{-\left(k-n_{p}\right)^{2} / 2 n p(1-\rho)}
$$

for $k$ in the $\sqrt{n p(1-p)}$ neighbourhood of $n p$. If the bit errors are independent, $n p \approx 100$ errors are required to ensure that the approximation is valid.

For the fading channels of interest, the bit errors are not independent (a single fade may cause several bit errors). Similarly. when convolutional coding is utilized, decoded bit 
errors tend to come in bursts. In both of these cases, approximately 100 independent error events are required. To aid in identifying the error event properties of a simulation run, the simulation program keeps track of the length and number of error-free runs. At lower bit error rates (e.g., $<10^{-2}$ ). this information can be used to estimate the number of error events. For example, if an enor event is defined to be a run of enrors terminating in at least 9 error free bits. then the number of error events is the number of runs of $\geq 9$ error free bits, minus one. 


\section{References}

[1] S. Chia, "The Universal Mobile Telecommunications System". IEEE Communications Magazine. Vol. 30. No. 12. pp. 54-62. December 1992.

[2] E. Reinhart. R. Taylor. A. Heyward and J. Miller. "WARC's last act?". IEEE Spectrum, Vol. 29. No. 2. pp. 20-33, February 1992.

[3] A. Viterbi. "Wireless Digital Communication: A View Based On Three Lessons Learned", IEEE Communications Magazine, Vol. 29. No. 9, pp. 33-36, September 1991.

[4] J. Anderson. T. Aulin and C.-E. Sundberg, Digital Phase Modulation. Plenum Press, New York, 1986.

[5] T. Aulin and C.-E. Sundberg, "Continuous Phase Modulation, Part I: Full Response Signaling", IEEE Transactions On Communications, Vol. COM-29. No. 3. pp. 196-209. March 1981.

[6] T. Aulin and C.-E. Sundberg, "Continuous Phase Modulation. Part II: Partial Response Signaling", IEEE Transactions On Communications, Vol. COM-29. No. 3. pp. 210-225. March 1981.

[7] J. Anderson and C.-E. Sundberg, "Advances in Constant Envelope Coded Modulation", IEEE Communications Magazine, Vol. 29, No. 12, pp. 36-45. December 1991. 
[8] K. Murota and K. Firade, "GMSK Modulation for Digital Mobile Radio Telephony". IEEE Transactions On Communications, Vol. 29, No. ?, pp. 1044-1050, July 1981.

[9] W. Stutzman, "Prolog to The Special Section on Propagation Effects on Satellite Communication Links". Proceedings Of The IEEE, Vol. 81. No. 6. pp. 850-855, June 1993.

[10] W.Y.C. Lee, Mobile Communications Engineering. McGraw-Hill. New York. 1982.

[11] J. Hagenauer and P. Hoeher, "A Viterbi Algorithm With Soft-Decision Outputs And Its Applications", Proceedings Of GLOBECOM '89. Dallas. Texas, pp. 47.11-47.17. November 1989.

[12] L.R. Bahl. J. Cocke. F. Jelinek, and J. Raviv, "Optimal Decoding Of Linear Codes For Minimizing Symbol ETror Rate", IEEE Transactions On Information Theon; Vol. IT-20. No. 2, pp. 284-287. March 1974.

[13] G.D. Forney Jr., "The Viterbi Algorithm", Proceedings Of The IEEE, Vol. 61. No. 3. pp. 268-278. March 1973.

[14] J. Hagenauer, "Soft-In / Soft-Out - The Benefits Of Using Soft Values In All Stages Of Digital Receivers", Third International Workshop On Digital Signal Processing Techniques Applied To Space Communications. September 1992.

[15] J. Lodge. R. Young. P. Hoeher and J. Hagenauer, "Separable MAP 'Filters' For The Decoding Of Product And Concatenated Codes". Proceedings of the International Conference on Communications (ICC'93), Geneva, Switzerland, pp. 1740-1745. May 1993.

[16] J. Lodge, R. Young. and P. Guinand. "Separable Concatenated Codes With Iterative MAP Filtering", Information Theory and Applications: Third Canadian Workshop. pp. 223-240, Springer-Verlag. 1994. 
[17] M. Moher. “Decoding via Cross-entropy Minimization". Proceedings Of GLOBECOM '93. Houston. Texas. pp. SO9-\$13. November 1993.

[1S] W.C. Jakes. Jr.. Microwave Mobile Communications. Wiley, New York. 1974.

[19] B. Sayer and S. Pasupathy. "Nyquist 3 Pulse Shaping In Continuous Phase Modulation". IEEE Transactions On Communications. Vol. COM-35. pp. 57-67. January 1987.

[20] J. Makhoul. "Linear Prediction: A Tutorial Review". Proceedings Of The IEEE. Vol. 63, pp. 561-580. April 1975.

[21] J.G. Proakis. Digital Communications, McGraw-Hill. New York, 1983.

[22] R.J. Young. Differential Detection With Channel Estimation Enhancement. M.Eng. thesis, Department of Systems and Computer Engineering. Carleton University. Ortawa, Canada. 1992.

[23] D. Divsalar and M.K. Simon. "Multiple Symbol Differential Detection of MPSK", IEEE Transactions On Communications, Vol. 38. No. 3. pp. 300-308. March 1990.

[24] D. Divsalar and M.K. Simon, "Maximum-Likelihood Differential Detection of Uncoded and Trellis Coded Amplitude Phase Modulation over AWGN and Fading Channels - Metrics and Performance". IEEE Transactions On Communications. Vol. 42, No. 1. pp. 76-89, January 1994.

[25] S. Crozier and R. Young, "Low Complexity Non-Coherent Multi-Symbol Detector for DMPSK Signals with Maximum Likelihood Performance", Proceedings of the International Conference on Wireless Communications (Wireless'93), Calgary, Alberta. pp. 367-375, July 1993. 
[26] K. Mackenthun, "A Fast Algorithm for Maximum Likelihood Detection of QPSK or $\pi / 4-Q P S K$ Sequences with Unknown Phase", 3rd International Symposium on Personal, Indoor and Mobile Radio Communications, Boston, Míassachusetts, pp. 240-244, October 1992.

[27] M. Moher and J. Lodge. "TCMP - A Modulation and Coding Strategy for Rician Fading Channels". IEEE Joumal On Selected Areas In Communications, Vol. 7. No. 9. pp. 1347-1355, December 1989.

[28] J. Cavers. "An Analysis of Pilot Symbol Assisted Modulation for Rayleigh Fading Channels". IEEE Transactions On Vehicular Technology; Vol. 40. No. 4, pp. 686-693, November 1991.

[29] A. Aghamohammadi. H. Meyr and G. Ascheid, "A New Method for Phase Synchronization and Automatic Gain Control of Linearly Modulated Signals on Frequency-Flat Fading Channels", IEEE Transactions On Communications. Vol. 39. No. 1.pp. 25-29. January 1991.

[30] C-L. Liu and K. Feher. "Pilot-Symbol Aided Coherent M-ary PSK in FrequencySelective Fast Rayleigh Fading Channels“, IEEE Transactions On Communications, Vol. 42. No. I. pp. 54-62. January 1994.

[31] J. McGeehan and A. Bateman. "Phase Locked Transparent Tone-In-Band (TIIB): A New Spectrum Configuration Particularly Suited To The Transmission Of Data Over SSB Mobile Radio Networks". IEEE Transactions On Communications, Vol. 32.pp. 81-87. January 1984.

[32] F. Davarian. "Mobile Digital Communications Via Tone Calibration", IEEE Transactions On Vehicular Technology. Vol. 36. No. 2. pp. 55-62. May 1987.

[33] J. Lodge and M. Moher. "Maximum Likelihood Sequence Estimation Of CPM Signals Transmitted Over Rayleigh Flat-Fading Channels". IEEE Transactions On Communications, Vol. 38. No. 6. June 1990. 
[34] Inmarsat Aeronautical System Definition Manual. Module 1: System Description. Version 1.45. December 1995.

[35] Qualcomm Incorporated. Q0256 k=7 Multi-Code Rate Viterbi Decoder. Technical Data Sheet. June 1990.

[36] J. Cavers. "On the validity of the slow and moderate fading models for matched filter detection of Rayleigh fading signals". Car. J. Elect. \& Comp. Eng.. vol. 17. no. 4. 1992.

[37] C. Berrou. A. Glavieux and P. Thitimajshima. "Near Shannon Limit Error Correcting Coding And Decoding: Turbo Codes(1)". Proceedings of the International Conference on Communications (ICC'93). Geneva. Swizzerland. pp. 1064-1070, May 1993.

[38] P. Hoeher. "Advances in Soft-Output Decoding". Proceedings Of GLOBECOM 93. Houston. Texas, pp. 793-797. November 1993.

[39] C. Nill and C.-E. Sundberg. "Viterbi Algorithms with List and Soft Symbol Outpur: Extensions and Comparisons". Proceedings Of GLOBECOM '93. Houston, Texas, pp. 788-792. November 1993.

[40] N. Seshadri and P. Hoeher. "On Post-Decision Symbol-Reliability Generation”. Proceedings of the Intermational Conference on Communications (ICC'93). Geneva. Switzerland. pp. 741-745. May 1993.

[41] D. Boudreau and Y. Viens. "Reduced Complexity Soft-Output Maximum Likelihood Sequence Estimation of 4-ary CPM Signals Transmitted over Rayleigh Flat-Fading Channels", submitted to IEEE Trans. Commun.. 1996.

[42] Q. Dai and E. Shwedyk, "Detection of bandlimited signals over frequency selective Rayleigh fading channels", IEEE Trans. Commun., vol. 42. pp. 941-950. Feb_Mar/Apr. 1994. 
[43] X. Yu and S. Pasupathy, "Innovations-based MISE for Rayleigh fading channels", IEEE Trans. Commun., vol. 43. pp. 1534-1544, Feb_Mar/Apr. 1995.

[4.4] R. Scheaffer and J. McClave, Probability and Statistics for Engineers, Duxbury Press. Boston, 1986. 